\title{
Molecular and structural correlates of ocular dominance plasticity in mice
}

\author{
Dissertation \\ for the award of the degree \\ "Doctor rerum naturalium" \\ of the Georg-August-Universität Göttingen \\ within the doctoral program IMPRS Neuroscience \\ of the Georg-August University School of Science (GAUSS)
}

submitted by

Rashad Yusifov

from Baku, Azerbaijan

Göttingen, April 2020 


\section{Examination Board}

Thesis advisory committee:

Prof. Dr. Siegrid Löwel

( $1^{\text {st }}$ referee)

Department of Systems Neuroscience, Johann-Friedrich-Blumenbach Institute for Zoology and Anthropology, University of Göttingen;

Campus Institute for Dynamics of Biological Networks, University of Göttingen

\section{Dr. Dr. Oliver Schlüter}

( $2^{\text {nd }}$ referee)

Department of Psychiatry and Psychotherapy, University Medical Center Göttingen;

Department of Neuroscience, University of Pittsburgh, USA

\section{Prof. Dr. Marion Silies}

Institute of Developmental Biology and Neurobiology, Johannes Gutenberg University Mainz

\section{Extended committee:}

Prof. Dr. Jochen Staiger

Institute for Neuroanatomy, University Medical Center Göttingen

\section{Dr. Katrin Willig}

Max Planck Institute of Experimental Medicine, Göttingen

\section{Prof. Dr. Florentin Wörgötter}

Third Physics Institute, University of Göttingen;

Bernstein Center for Computational Neuroscience, Göttingen 


\section{Affidavit}

I hereby declare that this thesis has been written independently and with no other sources or aids than quoted.

Rashad Yusifov

Göttingen, 30.04.2020 


\section{Table of Contents}

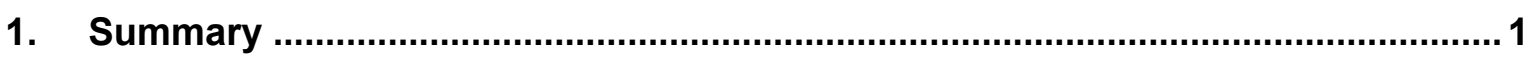

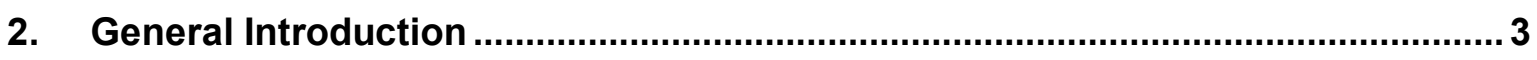

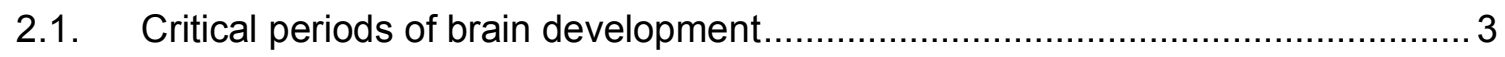

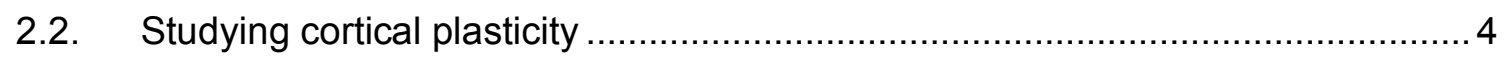

2.2.1. Physiological correlate: ocular dominance plasticity ................................ 5

2.2.2. Anatomical correlate: dendritic spine dynamics...................................... 8

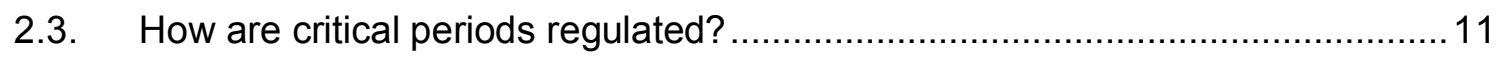

2.3.1. Maturation of inhibitory circuits........................................................... 11

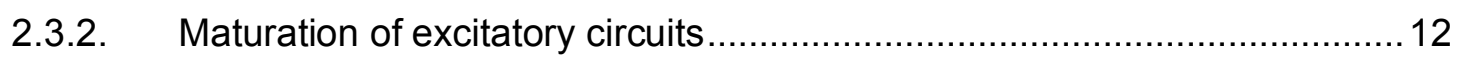

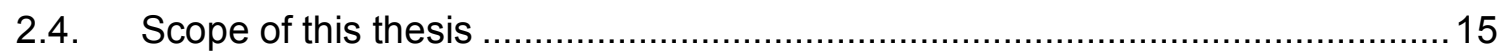

3. Experience-dependent spine dynamics in the visual cortex of awake PSD-95-

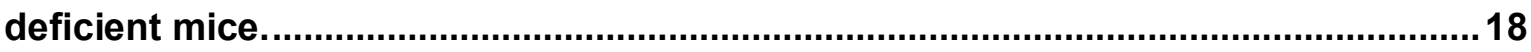

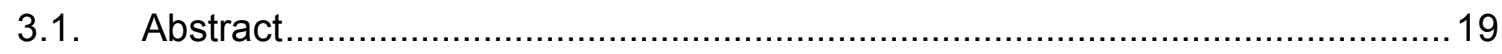

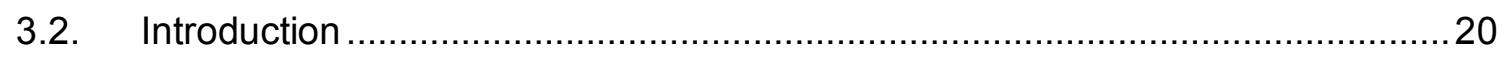

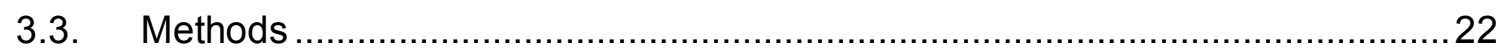

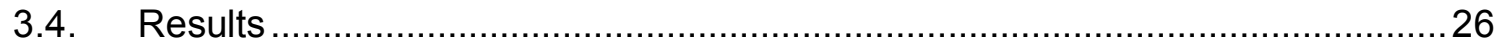

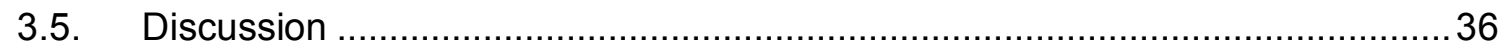

4. Loss of neurogranin in the visual cortex preserves juvenile-like ocular dominance plasticity into adulthood. .................................................................... 40

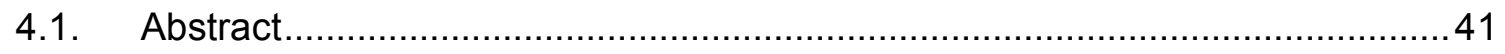

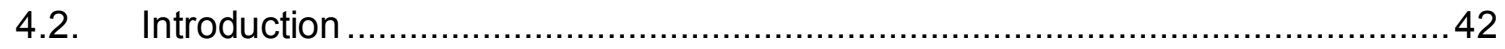

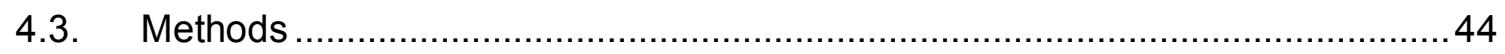

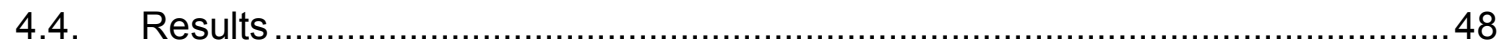

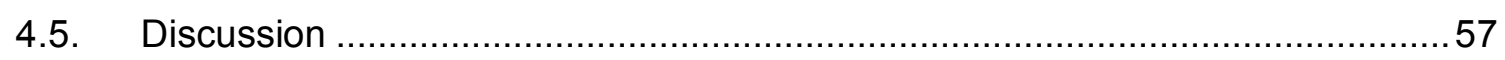

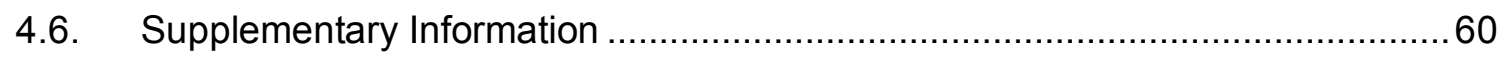

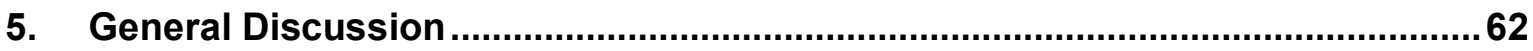

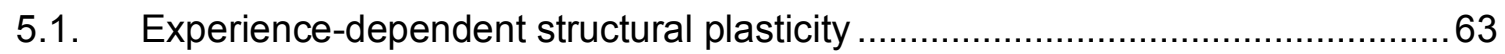

5.2. Recovery from monocular deprivation .................................................. 70

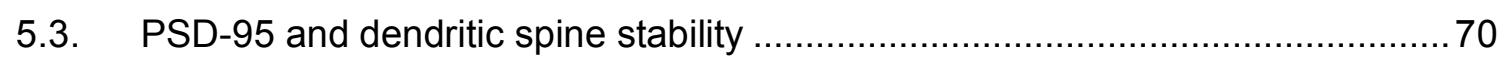


5.4. "Plasticity brakes" and dendritic spine stability .....

5.5. Role of neurogranin in the critical period closure for ODP and visual acuity .....74

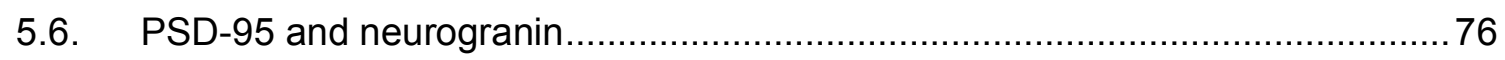

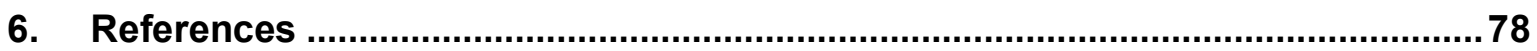

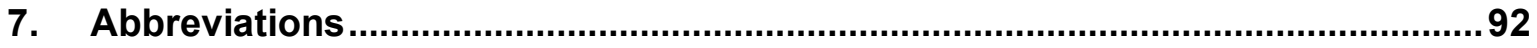

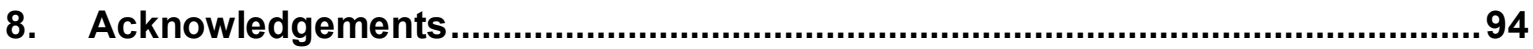

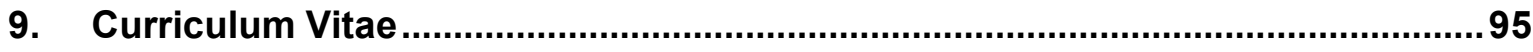




\section{Summary}

During early postnatal life, the brain goes through critical periods of increased plasticity, which enable experience-dependent refinements of neuronal circuits. Insertion of a-amino3-hydroxy-5-methyl-4-isoxazolepropionic acid receptors (AMPAR) to the post-synaptic membrane of nascent AMPAR-silent synapses during the critical periods is an essential neurodevelopmental process, enabling experience-dependent strengthening of favored glutamatergic connections. One of the key molecules that promotes AMPAR-silent synapse maturation is postsynaptic density (PSD) protein-95. It was recently shown that deleting PSD-95 halts the silent-synapse maturation at a developmentally immature state and prevents the closure of critical period for ocular dominance plasticity (ODP), a classical paradigm for studying the cortical plasticity (Wiesel and Hubel, 1963b). In adult mice lacking PSD-95, monocularly depriving the contralateral eye of the visual input for 4 days leads to a strong reduction in deprived-eye responses in the binocular part of the primary visual cortex (V1), and the plastic changes are reversed within 2 days of reopening the eye (Huang et al., 2015) - a phenotype classically seen only in juvenile wild-type mice if reared in standard conditions (Espinosa and Stryker, 2012).

In order to understand the underlying morphological correlates of enhanced cortical plasticity in the absence of PSD-95, I set out to investigate experience-dependent dendritic spine dynamics in layer 2/3 pyramidal neurons of binocular V1 in awake PSD-95 knockout (KO) and knockdown (KD) mice using awake, in-vivo two-photon imaging. Layer 2/3 pyramidal neurons in V1 were selectively transfected to express green fluorescent protein using in utero electroporation at embryonic day 15.5. Surgically implanting a long-term cranial window over V1 enabled repeated optical access to the superficial layers of the cortex. I imaged the same set of apical dendrites of layer 2/3 pyramidal neurons repeatedly, during and after a 4-day monocular deprivation (MD) of the contralateral eye in postnatal day $(P) \sim 75$ animals. Analyses of spine formation and elimination rates during these phases revealed that in PSD-95 KO mice, 4-day MD perturbs spine dynamics towards significantly increased spine elimination and a relative decrease in spine formation, while in WT controls spine dynamics remained essentially unchanged. The MD-induced imbalance in spine dynamics led to a significant reduction in spine density in PSD-95 KO mice. Interestingly, although the percentage of persistent spines was similar in KO and WT mice over the days of imaging, in PSD-95 KO mice newly formed spines were significantly more likely to be eliminated after MD. During the subsequent recovery phase, spine elimination ratio remained elevated in PSD-95 KO mice compared to baseline, although it was not anymore significantly different compared to WT controls. Similarly, in PSD-95 KD neurons MD induced a significant increase in spine elimination compared to controls, consistent with 
cell-autonomous impact of PSD-95 on AMPAR-silent synapse fractions. Overall, in PSD95-deficient mice, MD-dependent changes on spine dynamics were similar to that of previously described in juvenile mice (Sun et al., 2019), suggesting that absence of PSD95 results in juvenile-like plastic state.

Neurogranin, a postsynaptic signaling protein, was recently shown to also promote the AMPAR-silent synapse maturation and experience-dependent spine elimination during the time frame of critical period for ODP (Han et al., 2017). However, its role in critical period closure had not been investigated. Due to the elevated fraction of AMPAR-silent synapses in neurogranin-deficient mice, we hypothesized that the critical period for ODP should also remain open in these mice, similar to what has been observed previously in PSD-95deficient mice (Huang et al., 2015). Using optical imaging of intrinsic signals to assess ODP, here I show that knockdown of neurogranin at birth indeed i) prevented the closure of the critical period for ODP as observed for standard-cage raised mice (Gordon and Stryker, 1996), and ii) enhanced ODP even in mice up to at least P149, copying the phenotype previously observed in PSD-95-deficient mice. Moreover, while innate visual capabilities of adult neurogranin knockdown mice remained intact, their visual acuity was impaired, which is phenotypically different from PSD-95 deficient mice (Han et al., 2017; Huang et al., 2015). Thus, neurogranin is required for both the closure of the critical period for ODP and the functional development of $\mathrm{V} 1$ circuitry for optimal performance.

Overall, the findings in this thesis further substantiate the idea that enhanced brain plasticity during the early life is closely linked to the availability of AMPAR-silent synapses. Perturbing the molecules regulating the AMPAR-silent synapse maturation in the primary visual cortex, confers the cortical circuitry juvenile-like ODP plasticity, both structurally and physiologically. 


\section{General Introduction}

In his 1894 Croonian lecture of the Royal Society of London, Santiago Ramón y Cajal was describing the brain as composed of discrete cells that contact each other via dendritic protrusions, which he defined as spines (or "la espina" in his native Spanish). He even went further to postulate that mental processes such as learning and memory could be mediated by addition or removal of dendritic spines (Cajal, 1894). Although these were quite radical proposals on that day, it is hard to think of the brain otherwise now. Plasticity of the brain broadly refers to changes taking place during brain development, but also to its capacity to learn and adapt to new environments throughout life. Thus, one could even argue that plasticity of the brain is one of its utmost functions in ensuring the adaptability and, consequently, survival of the organism in ever changing surroundings. Modern neuroscience has long focused on understanding how such processes are accomplished in the brain. We now know that during the course of development, the brain goes through stages of heightened plasticity that are critical for shaping its function. Studying the molecular, physiological and anatomical changes during brain development reveals that processes involved in regulating brain plasticity, often go awry in neurodevelopmental disorders such as schizophrenia and autism. This thesis is devoted to further investigate those processes by focusing on the function of two important synaptic proteins, namely postsynaptic density (PSD) protein-95 (Cho et al., 1992; Stathakis et al., 1997) and neurogranin (Baudier et al., 1989), which are both closely implicated in the aforementioned disorders (Bourgeron, 2015; Hall et al., 2015; Ruano et al., 2008).

\subsection{Critical periods of brain development}

Initial neural wiring of the brain is laid out under the control of genetic factors for innate functionality. During the course of early development these connections get further refined and sculpted under the instruction of spontaneous activity patterns and normal sensory experience. As the brain becomes progressively more mature and less plastic, the capacity for such refinements gets gradually diminished. In humans, language learning is one of the commonly known examples of this phenomenon. Enhanced plasticity of brain before reaching the age of puberty enables a more native-like acquisition of a second language (Johnson and Newport, 1989). In contrast, adults learn languages differently, and a nativelike articulation in the second languages learned after puberty is seldomly achieved (Flege et al., 1995). Such time windows of experience-dependent refinement and heightened plasticity are termed as "critical periods" of the respective functional domain. Although first described experimentally in the visual cortex of young kittens by Drs. David Hubel and Torsten Wiesel (Wiesel and Hubel, 1963b), documented examples of the critical periods 
are widespread across various sensory and cognitive modalities, such as filial imprinting in several bird species (Lorenz, 1958), zebra finch song learning (Böhner, 1990), and barn owl auditory localization system (Knudsen et al., 1982; Knudsen and Konishi, 1979).

Another striking feature of critical periods is that the lack of sensory experience or aberrant experience during this sensitive phase of brain development leads to sometimes irreversible impairments in functionality of the respective domain, as described in the following examples. Songbirds need to listen to their song tutors of the same species during the respective critical period in order to learn singing; otherwise, they will only acquire a rudimentary song (Brainard and Doupe, 2002). Children with congenital cataracts may suffer from long-term visual acuity loss even after the cataracts are surgically removed (Birch, 2013; Webber and Wood, 2005). Recovery from such neurodevelopmental maladaptation or neurological injuries during adulthood requires enhancing the plasticity of brain. Thus, investigating how cortical plasticity is expressed in young and adult brains is of great importance. Uncovering the molecular and cellular processes that govern the duration of critical periods may open new therapeutic or pharmacological avenues to enhance or reinstate the juvenile plasticity in adult brain for the functional recovery of brain in cases of disease or trauma.

\subsection{Studying cortical plasticity}

Although the developmental plasticity of the brain had been postulated long before (Cajal, 1894), robust methodologies for experimentally studying and quantifying the plastic changes in the cortex have come to existence in 1960s. This was when Hubel and Wiesel published their initial papers describing electrophysiological recordings in the primary (striate) visual cortex of kittens (Hubel and Wiesel, 1962, 1963; Wiesel and Hubel, 1963b). These pioneering studies established that depriving young kittens of vision in one eye induces measurable physiological and anatomical changes in the neurons of the visual pathway .(Wiesel and Hubel, 1963a, b). This paradigm of monocular deprivation, as an experimental model of a cataract in human vision, quickly turned into a model to quantitatively study cortical plasticity in vivo. Thus began the decades of cortical plasticity research and has grown steadily as new and more sophisticated techniques were added to the toolbox of researchers. In the next sections, I will expand on how cortical plasticity can be measured both physiologically and anatomically. 


\subsubsection{Physiological correlate: ocular dominance plasticity}

In their pioneering experiments, Hubel and Wiesel (1962) found that individual neurons in the cat striate cortex respond preferably to visual stimuli that have certain orientation and are within certain visual field. Majority of the cortical neurons were activated by stimulation of either eye; however, some neurons were driven better by one eye stimulation over the other, giving rise to the term "ocular dominance" (OD). Furthermore, they observed that neighboring cells in V1 with similar orientation preference and OD properties are organized in radial columns extending through all layers of the cortex. In young kittens, suturing the contralateral eye led to a dramatic decrease in cortical responsiveness to the deprived eye stimulation (Wiesel and Hubel, 1963b), shifting the OD of neurons towards the open ipsilateral eye already after 3-4 days of deprivation (Hubel and Wiesel, 1970). They also showed that susceptibility to such plastic changes does not extend to the older animals, establishing that there is a critical period for MD-induced ocular dominance plasticity (ODP) (Wiesel and Hubel, 1963b). Detailed understanding of the ODP expression mechanism in juvenile animals came from the chronic single unit recordings in kittens (Mioche and Singer, 1989), where it was shown that, already 6-24h after MD, there is a decreased responsivity to deprived contralateral eye stimulation while open-eye responses remain stable. Later, ODP was also observed in macaque monkeys (Blakemore et al., 1978; Hubel et al., 1977), ferrets (Issa et al., 1999), rats (Rothblat et al., 1978) and finally mice (Dräger, 1978). Thus, the ODP paradigm became a readily available tool to study cortical plasticity in various experimental animals. Availability of variety of genetic tools and the shorter life span of mice has made the mouse visual system an especially attractive model for investigating the molecular and cellular mechanisms of brain plasticity during the critical period and beyond (Espinosa and Stryker, 2012).

\section{Mouse visual system}

The basic organization of the mouse visual system is largely similar to other mammals. Visual stimuli reaching the eyes get transformed into neuronal signals by the photoreceptors in the retina and are transmitted to the retinal ganglion cells through multilayered retina. The axons of retinal ganglion cells form the optic nerve and carry these signals to the lateral geniculate nucleus (LGN) of the thalamus and several other subcortical targets, such as superior colliculus. The main route for the visual information to reach cortex is the retinogeniculo-cortical pathway (Busse, 2018). Fibers carrying information from the nasal hemiretina ( $\sim 80 \%$ of total) cross to the contralateral side at the optic chiasm, and synapse onto dorsal lateral geniculate neurons on the contralateral side, which in turn send axons to 
the primary visual cortex (V1) through the optic radiation. Whereas fibers from the temporal hemiretina $(\sim 20 \%)$ do not cross at the optic chiasm and project to the ipsilateral LGN (Dräger and Olsen, 1980) (Fig. 1). Thus, V1 in each cortical hemisphere gets input from both eyes; however, the major input comes from the contralateral eye.

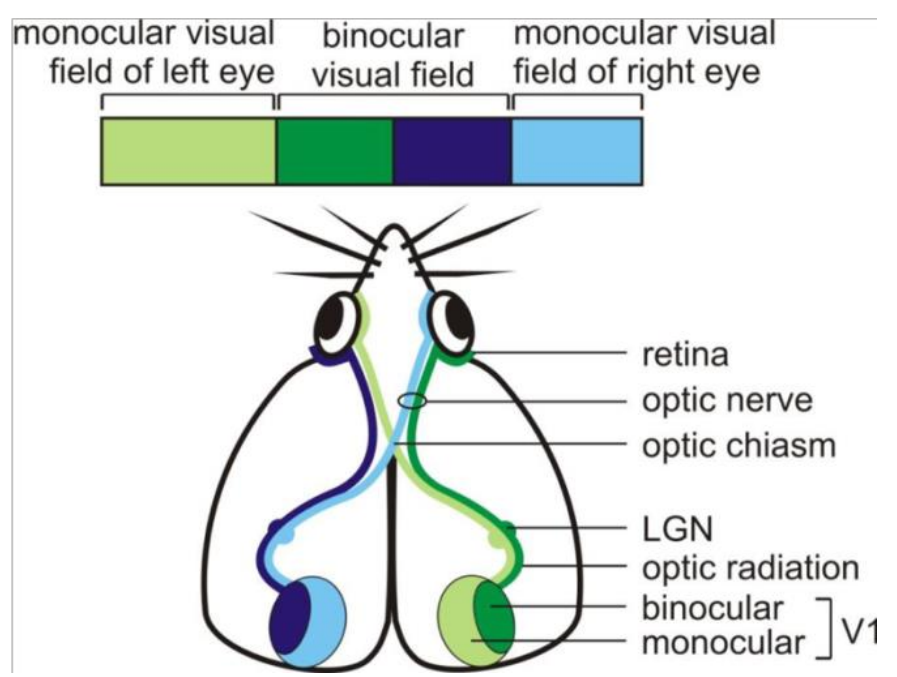

Figure 1. Central visual pathways of a C57BI/6J mouse [Figure modified from Greifzu et al. (2012)]. Organization of retino-geniculo-cortical visual pathway carrying visual information from the left (green) / right (blue) binocular (darker colors) and monocular (lighter colors) visual fields. Optic nerve fibers carrying information from the nasal hemiretina cross to the contralateral side at the optic chiasm and synapse onto dorsal lateral geniculate neurons (LGN) on the contralateral side, which in turn send axons to the primary visual cortex (V1) through the optic radiation. Fibers from the temporal hemiretina (input from binocular visual field of the eye) do not cross at the optic chiasm and project to the ipsilateral hemisphere (Dräger and Olsen, 1980).

\section{ODP in mice}

Mouse V1 lacks cortical orientation and ocular dominance columns seen in cats and macaque monkeys (Hubel and Wiesel, 1962; Hubel et al., 1976). It consists of a monocular area receiving input from only the contralateral eye and binocular area receiving input from both eyes (Dräger, 1975). In the binocular V1, the majority of neurons respond to the contralateral eye stimulation more strongly and thus exhibit contralateral OD (Dräger, 1975). In juvenile mice, binocular V1 undergoes rapid plastic changes after a brief (3-4 day) MD, shifting the OD from contra- to ipsilateral eye (Dräger, 1978), similar to what has been reported in kittens (Mioche and Singer, 1989). Such OD-shifts are mediated by a reduction 
in responsivity to the contralateral eye stimulation (Dräger, 1978). The critical period for ODP in mouse is between postnatal days (P)19 to P32 (Gordon and Stryker, 1996). Beyond the critical period for ODP (>P35), 4-d MD does not lead to significant OD-shifts in mice raised in standard-cage conditions (Gordon and Stryker, 1996). Nevertheless, the end of critical period is by no means an abrupt termination of the brain's plastic potential, as evidenced by experiments wherein a prolonged (7-day) MD could still result in OD-shifts in mice aged up to P110 (Lehmann and Löwel, 2008), and beyond P110 if the MD is further extended (Hosang et al., 2018). Importantly, the mechanism and kinetics of ODP expression is different in juvenile and adult animals. In juvenile-like ODP, 2-3 days after the $M D$, a reduction in deprived eye responses is observed, which is then followed by an increase in open-eye responses if the MD is prolonged (Frenkel and Bear, 2004). In adult mice, however, a longer (7-d) MD is needed to induce ODP (Lehmann and Löwel, 2008), and the OD-shifts are mediated by an increase in open eye responses (Sato and Stryker, 2008; Sawtell et al., 2003). The studies mentioned above have extensively characterized the ODP in young and adult mice, making it a reliable method of studying the cortical plasticity physiologically (Espinosa and Stryker, 2012).

Moreover, various experimental techniques, such as single unit recordings, visually evoked potentials, two-photon calcium imaging and optical imaging of intrinsic signals, can be utilized to assess the ODP at the single cell or population levels. Among these, optical imaging of intrinsic signals has the advantage of being the least surgically invasive, but lacks cellular resolution (Cang et al., 2005a; Kalatsky and Stryker, 2003). Optical imaging of intrinsic signals is based on the principle that active cortical regions consume more oxygen leading to a local accumulation of deoxyhemoglobin, which in turn absorbs more of the light that is shone onto the surface of skull and can be recorded by a highly sensitive camera after visual stimulation of either eye. Visual cortical maps are then calculated from the acquired frames by performing a Fourier analysis to extract the signal at the stimulation frequency (Cang et al., 2005a; Kalatsky and Stryker, 2003). Pixel intensities of resulting images are assessed to obtain the magnitude of $\mathrm{V} 1$ activation after deprived or spared eye stimulation, which then enables the experimenter to calculate the ocular dominance index (ODI) of binocular V1. Changes in ODI or V1 activation in animals with or without MD is analyzed to detect differences in cortical plasticity between control and experimental groups (Greifzu et al., 2012) (Fig. 2). 

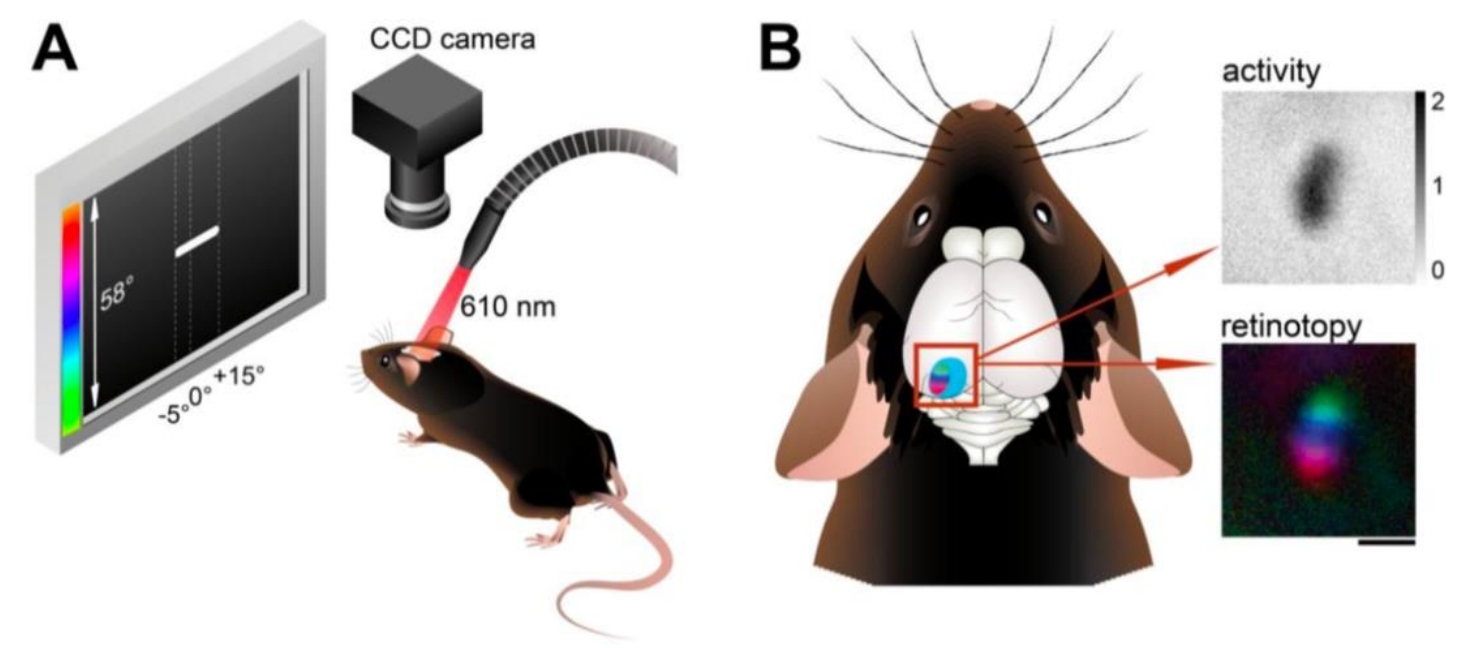

Figure 2: Visualization of neuronal activity using optical imaging of intrinsic signals [Figure modified from Greifzu et al. (2012)]. (A) A white horizontal bar moving upwards or downwards on a black background is presented to an anesthetized mouse. The stimulus activates the neurons in the binocular part of V1. Increased neuronal activity leads to local accumulation of deoxyhemoglobin, which absorbs more of the red light $(610 \mathrm{~nm})$ shone onto cortex. Resulting changes in light reflectance are recorded using a light-sensitive CCD camera and are extracted by Fourier analysis. (B) An activity map (top) and retinotopic polar map (bottom) of the binocular zone of $\mathrm{V} 1$. The color-coded retinotopic map represents the neuronal response depending on the spatial position of the stimulus in the visual field. Scale bar: $1 \mathrm{~mm}$.

\subsubsection{Anatomical correlate: dendritic spine dynamics}

We have come a long way in visualizing the synaptic structures since the first camera lucida drawings of Golgi stained neurons of Santiago Ramón y Cajal (Cajal, 1888). Although during his lifetime Cajal already postulated that the small protrusions on dendrites are the sites of contact between neurons (Garcia-Lopez et al., 2007), visualizing synapses directly has only become possible after the invention of electron microscopy (EM) by Ernst Ruska in 1931 (Gray, 1959; Ruska and Knoll, 1931; Uchizono, 1965). Early EM studies have established the existence of almost one to one correlation between dendritic spines and excitatory synapse existence (Harris et al., 1992; LeVay, 1973). Later, immuno-EM also showed that glutamate-positive boutons make connections only with proteinaceous postsynaptic density (PSD)-containing partners, which were mostly located on dendritic spines (DeFelipe et al., 1988). Thus, dendritic spines became the site of interest for the 
future investigations of excitatory transmission. Nevertheless, in the following decade visualizing the neuronal structures was limited to investigating fixed slices of brain tissue, which only allowed static analyses of brain anatomy (Berry and Nedivi, 2017). Plastic changes accompanying brain development or sensory restrictions or manipulations could only be studied at the population level by fixing the samples at various ages or following sensory manipulations. Although fixed tissue investigations revealed large-scale structural reorganizations, the subtle changes in dendritic spines or axonal boutons could easily be overlooked (Berry and Nedivi, 2017). Later, expression of fluorescent proteins (Chalfie et al., 1994) and fluorescent dye injections (Dailey and Smith, 1996) in the cultured cells or brain slices allowed imaging of individual spines longitudinally, albeit for only a limited time window and ex vivo. These studies started to reveal the surprisingly dynamic nature of dendritic spines (Yuste and Bonhoeffer, 2001). Nevertheless, it was not until the advent of two-photon microscopy that researchers had the necessary technical means to image synaptic structures in the intact brain of living animals over an extended time window (Denk et al., 1990; Denk and Svoboda, 1997; Svoboda et al., 1997; Svoboda et al., 1996). The following two-photon studies dramatically expanded our understanding of how neural networks are assembled during development and modified with experience.

In vivo imaging of spines was also made possible by fluorescent labeling techniques such as the availability of transgenic mouse lines expressing fluorescent proteins (Feng et al., 2000), in utero electroporation (Saito and Nakatsuji, 2001) or virus injections (Jeromin et al., 2003). Use of transgenic mice line expressing yellow fluorescent protein (YFP) or GFP in a subset of layer 5 pyramidal neurons was possibly the most famous option among these during the early days of two-photon microscopy (Feng et al., 2000; Grutzendler et al., 2002; Holtmaat et al., 2005; Trachtenberg et al., 2002). In these studies, the apical dendrites of layer 5 pyramidal neurons passing through layer 1 were imaged through a chronically implanted cranial window or a thinned skull. Thus, most of the knowledge we gained about dynamics of spines is biased towards the apical dendrites of layer 5 pyramidal neurons in mice, although this is nowadays changing due to the use of other approaches such as inutero electroporation enabling also the specific labeling of neocortical layer $2 / 3$ cells (Chen et al., 2012; Sun et al., 2019; Villa et al., 2016). Importantly, the earlier studies already revealed that there are age, modality, and lamina specific differences in spine dynamics (Holtmaat et al., 2005; Trachtenberg et al., 2002). Consequently, it is crucial to consider these parameters when designing an experiment or comparing results between different studies. 
In mice, around P30, dendritic protrusions of layer 5 and 2/3 pyramidal neurons get rapidly added and eliminated, have higher motility, exhibit more filopodia-like shapes across the cortex (Grutzendler et al., 2002; Holtmaat et al., 2005; Majewska et al., 2006; Oray et al., 2004; Trachtenberg et al., 2002). Starting from adolescence (>P30), spine dynamics gradually slow down, and dendritic spines across sensory cortices and cortical layers acquire more mature shapes (Grutzendler et al., 2002; Holtmaat et al., 2005). However, even in fully mature mice (>P110), $\sim 10-30 \%$ of the spines in the visual and somatosensory cortices are still dynamic, meaning that they disappear and reappear within 4-days, while the rest are defined as persistent and can be stable for weeks and possibly longer (Grutzendler et al., 2002; Holtmaat et al., 2005). Interestingly, spines in the barrel cortex are more dynamic compared to the visual cortex (Holtmaat et al., 2005). Moreover, the apical dendrites of layer 2/3 neurons in barrel cortex have higher spine density, and the spines are much more stable in comparison to layer 5 (Holtmaat et al., 2005), although this has not yet been systematically addressed in the visual cortex. It is also important to consider that in vivo two-photon imaging studies have majorly focused on apical dendrites, while basal dendrites are located too deep in the tissue for achieving a good signal to noise ratio at the spine level with standard two-photon imaging protocols. In general, although there are some gaps that still need to be filled, it is now safe to say that the increased structural dynamics of dendritic spines during early life correlates well with the increased brain plasticity observed in physiological and behavioral studies, and both decline gradually during the development.

One major advantage of in vivo chronic imaging is that in addition to monitoring baseline dynamics, it also offers the possibility to visualize circuit rewiring in the intact brain of awake or anesthetized animals after sensory and motor manipulations, such as visual deprivation, whisker trimming or lever pressing (Berry and Nedivi, 2017). Admittedly, in most of the studies, these kinds of analyses are usually restricted to only a few cells or dendrites per animal in a particular cortical layer and cortical region due to time limitations, and thus are not yet able to visualize how the whole micro-circuit remodels. Since there are layer and region-specific differences between dendritic spine dynamics and how they respond to different manipulations, it is essential for the experimenter to define beforehand what will be the focus of the study to answer the scientific question at hand. In V1, plastic changes associated with juvenile ODP are thought to be expressed rapidly in supra- and infragranular layers of the cortex. It was already shown that a 3-d MD during the critical period for ODP leads to rapid spine elimination at the apical dendrites of layer $2 / 3$ and layer 5 PNs (Sun et al., 2019; Zhou et al., 2017), which correlates well with reduced deprived eye responses and the time scale of changes observed in physiological studies (Sun et al., 
2019). In contrast, during adulthood, MD induces spine formation in the apical dendrites of layer 5 PNs, while not affecting spine dynamics of layer 2/3 PNs (Hofer et al., 2009). A more drastic sensory manipulation such as unilateral retinal lesions can almost completely replace the existing dendritic spines with new ones on layer 5 apical dendrites (Keck et al., 2008). Notably, layer 2/3 inhibitory synapses in V1 become more dynamic after 6-d MD in adult mice (Villa et al., 2016). The combination of two-photon microscopy and the genetic tools now allows the researchers to dissect out the function of specific molecular components in regulating the aforementioned spine dynamics in naïve and sensorydeprived animals (Peebles et al., 2010; Sajo et al., 2016).

\subsection{How are critical periods regulated?}

During critical periods sensory experience is the major driving force for cortical refinement and leads to the maturation of both inhibitory and excitatory circuitry. Several additional processes, such as expression of extracellular factors that limit structural plasticity, epigenetic modifications, and neuromodulatory systems have also been shown to contribute to the developmental regulation of brain plasticity (Espinosa and Stryker, 2012; Hensch, 2005; Hensch and Quinlan, 2018; Levelt and Hübener, 2012; Stryker and Löwel, 2018). Interfering with these processes may impact the cortical plasticity at various developmental timepoints; however, the chain of events that lead to constricted time windows of critical periods and that allow experience dependent refinement during the critical period are not fully resolved. Accumulating evidence suggests that while inhibitory maturation is needed for the opening of critical period for ODP, maturation of excitatory transmission is instructive in closing it (Favaro et al., 2018; Huang et al., 2015).

\subsubsection{Maturation of inhibitory circuits}

In mice, experience-dependent refinement of the visual cortex begins at eye opening (P14) (Espinosa and Stryker, 2012). In V1, the ocular dominance of contralateral eye is established before eye opening, but the critical period for ODP does not start immediately at eye opening and is rather delayed until $\sim$ P20 in mice (Espinosa and Stryker, 2012). A series of experiments have established that the experience-dependent increase in inhibitory tone leads to the opening of the critical period for ODP in mouse V1. As such, perturbing $\gamma$ aminobutyric acid (GABA) production during the development has a striking effect on the onset of the critical period for ODP. Genetically modified mice lacking one of the GABAproducing enzymes, GAD-65 (glutamic acid decarboxylase, $65 \mathrm{kDA}$ ), have lower levels of GABA production and do not exhibit ODP (Hensch et al., 1998) - meaning that in these 
mice the CP for ODP does not open. The lack of ODP in GAD65 KO mice can be rescued by a brief diazepam infusion, which leads to opening of a critical period, similar in duration and quality to wild-type mice (Fagiolini and Hensch, 2000). However, it has to be noted that in GAD65 KO mice, the subunit composition of NMDA receptors is also affected, where GAD65 KO mice show reduced levels of NR2A subunits (Kanold et al., 2009). In V1 of WT animals, increased inhibition is paralleled by an experience-dependent increase in NR2A subunit expression during development (Carmignoto and Vicini, 1992; Quinlan et al., 1999). Thus, increased inhibition in the beginning of the CP is permissive for NMDAR-dependent plasticity. Notably, infusion of diazepam (at P15) before the natural opening of critical period leads to precocious opening of the critical period for ODP, which then lasts for 2 weeks as normally (Fagiolini and Hensch, 2000). It was later shown that the effect of diazepam in precociously opening the critical period is mediated through its binding to $\alpha 1$-subunit containing $\mathrm{GABA}_{\mathrm{A}}$ receptors (Fagiolini et al., 2004). As parvalbumin-expressing (PV) inhibitory cells make contacts to $\alpha 1$-subunit containing $\mathrm{GABA}_{A}$ synapses (Klausberger et al., 2002), PV cells are likely to be closely involved in the opening of the critical period for ODP.

GABA transmission continues to strengthen gradually during development (Hensch et al., 1998), while blocking the GABA production pharmacologically in the visual cortex was shown to enhance the ODP in adult rats. (Harauzov et al., 2010). However, it is not clear how increasing inhibition instructs the cortical refinement during CPs. A promoting role of sensory experience on inhibitory maturation has been shown to be exerted via brain-derived neurotropic factor (BDNF) (Gianfranceschi et al., 2003). Although BDNF expression was shown to promote the GABAergic innervation and increase inhibition (Huang et al., 1999), its effects are not limited to the maturation of inhibitory system, and may also function via promoting AMPAR-silent synapse maturation (Itami et al., 2003). The next section explores how the maturation of glutamatergic circuitry may offer an explanation for the progression and closure of critical periods.

\subsubsection{Maturation of excitatory circuits}

Experience-dependent maturation of glutamatergic transmission plays an essential role in governing the duration of critical periods (Favaro et al., 2018; Huang et al., 2015). Fast excitatory transmission in mature glutamatergic synapses happens mainly through two ion channels, namely $\mathrm{N}$-methyl-D-aspartate receptors (NMDARs) and $\alpha$-amino-3-hydroxy-5methyl-4-isoxazolepropionic acid receptors (AMPARs). During early cortical development, newly forming glutamatergic synapses are AMPAR-silent, denoting that they lack AMPARs, 
and transmit mainly via NMDARs (Isaac et al., 1995; Liao et al., 1995). At the resting membrane potential, miniature excitatory postsynaptic potentials result from the transmission at the AMPARs, while NMDAR are mostly blocked by $\mathrm{Mg}^{2+}$ ions. Hence, unless the synapse is depolarized and the $\mathrm{Mg}^{2+}$ block is removed, nascent synapses missing AMPARs are silent at the resting membrane potential and are thus termed as "AMPARsilent" synapses. They can be converted into AMPAR-transmitting synapses if the synapse undergoes long-term potentiation (LTP), as a result of AMPAR incorporation into the postsynaptic membrane (Isaac et al., 1995; Liao et al., 1995). AMPAR insertion and consequently LTP of the newly formed synapses are experience-dependent. At the age of eye opening in mice, in the visual cortex around $50 \%$ of the synapses in layer 4 (PN) to layer 2/3 circuit are AMPAR-silent (Han et al., 2017; Huang et al., 2015). Normal sensory experience leads to a progressive decline in AMPAR-silent synapse fractions by the end the of critical period for ODP (Favaro et al., 2018; Han et al., 2017; Huang et al., 2015). Mice reared in the dark from birth do not exhibit the developmental decline in silent synapse fractions, showing that sensory experience is essential for AMPAR-silent synapse maturation (Favaro et al., 2018; Funahashi et al., 2013). During the critical period, glutamatergic connections get strengthened putatively in use-dependent manner, whereas unused (or unfavored) connections get pruned to maintain the overall AMPAR-mediated transmission in equilibrium in an experience-dependent manner (Han et al., 2017). Abovementioned findings strongly imply that AMPAR-silent synapses provide synaptic opportunities for neurons to be wired together as instructed by the sensory experience. Once commenced, duration and termination of critical periods may thus depend on the maturational time course of such malleable synaptic connections.

Definitive evidence for the silent-synapse based regulation of critical periods comes from studying the molecular components of experience-dependent silent synapse maturation (Favaro et al., 2018; Huang et al., 2015). It was shown that two paralog membraneassociated guanylate kinases (MAGUKs) - postsynaptic density protein (PSD)-95 and PSD93 - regulate the critical period for ODP through a mechanism based on AMPAR-silent maturation. I will describe the role of these proteins in the following paragraphs while also summarizing the major results of the respective studies. These findings establish a causal link between AMPAR-silent synapse maturation and the critical period regulation for ODP in mouse $\mathrm{V} 1$, and also set the stage for the conception of the present thesis.

PSD-95 is a major signaling scaffold protein in the postsynaptic membrane of excitatory synapses in vertebrates and a part of the molecular cascade trafficking AMPAR to the postsynaptic membrane (Béïque et al., 2006; El-Husseini et al., 2000; Stein et al., 2003). In 
addition to trafficking, PSD-95 also indirectly stabilizes AMPARs in the postsynaptic membrane through the intermediary protein stargazin (Fukata et al., 2005). Absence of PSD-95 leads to $\sim 50 \%$ reduction in AMPAR-dependent transmission (Béïque et al., 2006; Elias et al., 2006; Nakazawa et al., 2001; Schlüter et al., 2006); impairs long-term depression (LTD) while facilitating LTP in mice hippocampal slices (Migaud et al., 1998), although the subsequent maintenance of LTP is also impaired in the absence of PSD-95 (Ehrlich et al., 2007). On the other hand, overexpression of PSD-95 occludes LTP, while LTD is facilitated (Béïque and Andrade, 2003; Stein et al., 2003). Thus, PSD-95 is an essential component of synaptic plasticity mechanisms.

Notably, the expression profile of PSD-95 protein in the visual cortex fits to that of the critical period of ODP: in mice, PSD-95 protein expression starts to increase at eye opening and reaches $\sim 80 \%$ of its peak expression level by the end of the critical period ( P35) (Huang et al., 2015; Yoshii et al., 2003). This also correlates with the developmental decrease of AMPAR-silent synapse numbers in the same time window (Favaro et al., 2018; Huang et al., 2015). In PSD-95 KO mice, AMPAR-silent synapse fractions remain elevated in the visual cortex during adulthood, and this prevents the closure of the critical period, resulting in preservation of juvenile-like ODP at least up to P480 (Huang et al., 2015). ODP in PSD$95 \mathrm{KO}$ mice resembles that of juvenile mice in three important ways: 1) only a brief (4-day) MD is necessary to induce strong OD shifts in mice aged up to P480;2) ODP is mediated by a reduction of optically recorded $\mathrm{V} 1$-responses to visual stimulation of the previously deprived eye, with no concomitant changes in V1-responses to the non-deprived, open eye; 3) plastic changes (OD-shifts) are transient, in that contralateral dominance of mice can easily be regained after reopening the deprived eye for only 2 days. Experiments with adeno-associated virus (AAV) mediated knockdown of PSD-95 in the visual cortex showed that the effects of PSD-95 on ODP are cortex specific. Moreover, knock-down of PSD-95 in the visual cortex of adult mice (>P40) restored the fractions of AMPAR-silent synapses to the eye opening levels in a cell-autonomous fashion and also reopened the critical period for ODP, thus establishing a causative link between AMPAR-silent synapse maturation and the termination of the critical period for ocular dominance plasticity in mouse V1 (Huang et al., 2015). Importantly, excitatory input onto PV cells and the inhibitory synapses onto layer 2/3 pyramidal neurons were not altered in PSD-95 KO mice, and diazepam treatment did not prevent ODP in adult PSD-95 KO mice, concluding that increased inhibitory tone is not required for closure of the critical period for ODP (Huang et al., 2015).

PSD-93 is a paralog of PSD-95, and is also involved in controlling the synaptic trafficking of AMPARs. Although there is some overlap in the functions of PSD-95 and PSD-93 as shown 
in mice hippocampal slice cultures (Elias et al., 2006), interestingly, PSD-93 inhibits PSD95 function in promoting the maturation of the AMPAR-silent synapses in pyramidal neurons (Favaro et al., 2018). Its loss results in a faster decline in AMPAR-silent synapse fractions and leads to a precocious closure of the critical period for ODP in mouse V1 (Favaro et al., 2018). Thus, there is a reverse and forward correlation between the AMPAR-silent synapse abundance and the critical period for OD. Moreover, if PSD-93 KO mice are dark reared, the fractions of AMPAR-silent synapses still decrease in an experience independent manner. Interestingly, in the absence of both paralogs, the maturation of silent synapses progressed similarly to WT mice and was not affected by dark rearing (Favaro et al., 2018). These results show that balanced functions of PSD-93 and PSD-95 are required for normal pacing of the experience-dependent AMPAR-silent synapse maturation.

Neurogranin is a postsynaptic signaling protein that has been recently demonstrated to also promote experience-dependent AMPAR-silent synapse maturation in mouse V1 (Han et al., 2017). It is enriched in the dendritic spines of principal neurons of cerebral cortex and hippocampus (Represa et al., 1990), and plays a key role in synaptic plasticity through controlling the spatiotemporal availability of calmodulin (CaM) and thus $\mathrm{Ca}^{2+} / \mathrm{CaM}$ dynamics (Gaertner et al., 2004). In mice, loss of neurogranin impairs LTP in hippocampal brain slices (Hwang et al., 2018), whereas enhances LTD in layer 4 to layer $2 / 3$ pyramidal neuron circuitry of V1 (Han et al., 2017). Similar to PSD-95 and PSD-93, neurogranin levels increase during early development and reach adult levels at P30, coinciding with the end of the critical period for ODP in mouse V1. However, its role in the regulation of the critical period for ODP had not been investigated in detail, and therefore constitutes a second major part of the present thesis.

\subsection{Scope of this thesis}

Previous results have established that PSD-95 is required for the experience-dependent closure of the critical period for ODP in mouse V1 through promoting the maturation of AMPAR-silent synapses (Huang et al., 2015). In PSD-95 KO mice, juvenile-like ODP is preserved at least up to P480, and visual cortex specific PSD-95 knockdown reopens the critical period in adulthood. Abundance of AMPAR-silent synapses in PSD-95-deficient mice correlates with a juvenile state of cortical plasticity (Huang et al., 2015). In the present study, I aimed at investigating whether such physiologically juvenile-like state in adult PSD$95 \mathrm{KO}$ animals also translates into enhanced structural plasticity at the single neuron level that is otherwise typically observed in juvenile mice. In order to be able to link the structural data to previously obtained physiological results, I visualized dendritic spine dynamics of 
layer 2/3 pyramidal neurons (PNs) in binocular V1 of awake mice using a custom built twophoton microscope. It is generally accepted that in juvenile mice, baseline spine dynamics is elevated compared to adulthood (Holtmaat and Svoboda, 2009). Recently, it was also shown that in juvenile mice, monocular deprivation (MD) leads to increased spine elimination in the apical dendrites of layer 2/3 PNs (Sun et al., 2019), while during adulthood, these spines are not affected even by a longer (8-d) MD (Hofer et al., 2009). We hypothesized that in the visual cortex of mature PSD-95-deficient mice, dendritic spines would exhibit more juvenile-like dynamics, anatomically correlating with their juvenile-like ODP phenotype.

To label the dendritic spines selectively in visual cortical layer $2 / 3$, I have performed in utero electroporation in PSD-95 WT and KO embryos on embryonic day 15.5, transfecting the progenitors of the layer $2 / 3$ PNs with an enhanced GFP expressing plasmid. Once the mice were beyond postnatal day 45 (>P45), i.e. clearly beyond the critical period for ODP, I have surgically implanted a chronic cranial window above $\mathrm{V} 1$, allowing me to perform in-vivo imaging of apical dendrites of layer 2/3 PNs longitudinally in the same animals. Mice were thoroughly trained for 2-3 weeks to get used to head-restraining so that spine dynamics could be imaged in the awake brain (during the two-photon imaging for $<1 \mathrm{hr}$ ), to avoid any potential effects of repeated exposure to anesthesia. Once trained, the same set of apical dendrites of PNs in binocular V1 of adult PSD-95 WT and KO mice was followed in seven imaging sessions (that spanned 12 days in total) during normal binocular vision, monocular deprivation and reopening of the previously deprived eye. It was shown previously that $\mathrm{V} 1$ specific knockdown of PSD-95 increases the fraction of AMPAR-silent synapses cell autonomously, and preserves a juvenile ODP into adulthood similar to PSD-95 KO mice (Huang et al., 2015). Thus, I have next imaged dendritic spine dynamics also in another experimental group of mice, in which PSD-95 was sparsely knocked down in layer 2/3 PNs by a construct expressing shRNA against PSD-95. The results obtained from the dendritic spine dynamics experiments are presented in the first manuscript. In summary, I show that PSD-95-KO or deficiency leads to a strongly increased experience-dependent spine dynamics of layer 2/3 PNs, quantitatively similar to what has been previously observed in juvenile mice (Sun et al., 2019; Zhou et al., 2017).

In the second part of the thesis, I have investigated the role of neurogranin in closing the critical period for ODP in mouse V1. Recently, it has been shown that neurogranin is closely implicated in the maturation of glutamatergic circuity, by coordinating experiencedependent AMPAR-silent synapse maturation and dendritic spine pruning during the critical period for ODP (Han et al., 2017). As the role of neurogranin seems to be similar to that of 
PSD-95 with respect to AMPAR-silent synapse maturation, we have hypothesized that silencing neurogranin expression in V1 would also preserve juvenile ODP into adulthood. Using intrinsic signal optical imaging (Cang et al., 2005a; Huang et al., 2015), I have assessed the ODP in V1 of control and neurogranin-knockdown mice belonging to two different age groups: 1 ) young adult mice (P49-83) and 2) an older group (P125-149). In summary, silencing neurogranin expression prevented the critical period closure for ODP and enhanced ODP even in the older group of mice. Knockdown of neurogranin also impaired visual acuity as tested by the visual water task. These results further support the idea that maturation of AMPAR-silent synapses regulate the duration of critical period for ODP. 


\section{Experience-dependent spine dynamics in the visual cortex of awake PSD-95-}

deficient mice.

Rashad Yusifov ${ }^{1,2}$, Anja Tippmann ${ }^{1}$, Jochen Staiger ${ }^{2,3}$, Oliver M. Schlüter ${ }^{2,4,5}$, Siegrid Löwel ${ }^{1,2,6}$

${ }^{1}$ Department of Systems Neuroscience, Johann-Friedrich-Blumenbach Institut für Zoologie und Anthropologie, Universität Göttingen, Göttingen, Germany

${ }^{2}$ Collaborative Research Center 889, Universität Göttingen, Göttingen, Germany

${ }^{3}$ Institute for Neuroanatomy, University Medical Center, Universität Göttingen, Göttingen, Germany

${ }^{4}$ Department of Neuroscience, University of Pittsburgh, Pittsburgh, PA, USA

${ }^{5}$ Department of Psychiatry and Psychotherapy, University Medical Center Göttingen, Göttingen, Germany

${ }^{6}$ Campus Institute for Dynamics of Biological Networks, Universität Göttingen, Göttingen, Germany

Author contributions of Rashad Yusifov:

- Study design (together with Siegrid Löwel, Oliver M. Schlüter, Anja Tippmann)

- Performance and analysis of the experiments shown in all the figures

- Interpretation of the results (together with Siegrid Löwel and Oliver M. Schlüter)

- Preparation of the manuscript

This manuscript has been prepared for publication. 


\subsection{Abstract}

Critical periods $(\mathrm{CP})$ are time windows of heightened brain plasticity and neural refinement during the early development. It was recently shown that duration of the $\mathrm{CP}$ for ocular dominance plasticity (ODP) is instructed by experience-dependent maturation of AMPARsilent synapses, and impairing this process by genetically removing postsynaptic density (PSD) protein-95 prevents the CP closure. In adult PSD-95-deficient mice a brief MD induces robust and juvenile-like ODP, and the changes are promptly recovered after reopening the deprived eye. Here we investigated the dendritic spine dynamics associated with enhanced ODP in adult PSD-95-deficient mice. Using a two-photon microscope, we repeatedly imaged the apical dendrites of layer $2 / 3$ pyramidal neurons in the binocular part of primary visual cortex (V1) before, during, and after a 4-day MD in awake animals. We found that before $M D$, spine formation and elimination rates were comparable between PSD-95 WT and KO mice. During 4-day MD, however, spine elimination rate in KO mice nearly doubled, while spine gain was relatively decreased, causing a significant reduction in spine density. Interestingly, newly formed spines were more likely to be eliminated in KO mice during the MD. After reopening the deprived eye, spine elimination and formation rates became comparable between PSD-95 WT and KO mice; however, in KOs spine elimination rate remained high compared to pre-MD values. Moreover, PSD-95 function in promoting spine stability was cell-autonomous. Overall, MD-dependent spine dynamics in adult PSD95-deficient mice were strongly reminiscent of juvenile mice, underscoring the role of PSD95 in cortical maturation. 


\subsection{Introduction}

Early life of an animal is characterized by time windows of enhanced brain plasticity known as critical periods (CP), which have been described for the first time in the primary visual cortex (V1) of young kittens (Wiesel and Hubel, 1963b), but also in several other functional domains across animal models (Brainard and Doupe, 2002; Feldman et al., 1996; Roberts et al., 2010). The CP for ocular dominance plasticity (ODP) in V1 is likely the most experimentally investigated among them (Dräger, 1978; Espinosa and Stryker, 2012; Hubel and Wiesel, 1963; Rothblat et al., 1978; Wiesel and Hubel, 1963b). During the CP for ODP, a brief monocular deprivation (MD) of the contralateral eye results in a shift in the ocular dominance (OD) towards the open eye through reduction in deprived eye responses (Dräger, 1978; Gordon and Stryker, 1996; Mioche and Singer, 1989). However, after the CP cortical plasticity declines progressively (Lehmann and Löwel, 2008). Heightened plasticity of the cortex in juvenile mice correlates with elevated baseline dynamics of cortical synaptic structures, which become progressively less dynamic during the development (Grutzendler et al., 2002; Holtmaat et al., 2005). Increased dynamics of cortical synapses in layer (L) 2/3 and L5 neurons have also been closely associated with sensory manipulations (Hofer et al., 2009; Holtmaat et al., 2006; Sun et al., 2019; Villa et al., 2016; Zhou et al., 2017), including MD [reviewed in (Berry and Nedivi, 2016)]. During the CP for ODP, a brief MD of the contralateral eye increases the rate of dendritic spine elimination on the apical dendrites of L5 PNs in the binocular V1 (Zhou et al., 2017). Moreover, it was recently shown that MD-induced shifts in the OD of individual L2/3 pyramidal neurons (PNs) are directly correlated to the magnitude of spine elimination in the apical dendrites (Sun et al., 2019).

Although several components involved in the experience-dependent maturation of cortical circuits have been described (Espinosa and Stryker, 2012; Hensch and Quinlan, 2018), the molecular chain of events leading to the closure of CPs remains elusive. We and others have previously shown that nascent synapses, containing NMDA-type but lacking AMPAtype glutamate receptors (AMPAR), defined as AMPAR-silent synapses (Isaac et al., 1995; Liao et al., 1995), can provide a well-fitting substrate for experience-dependent maturation of cortical neurons (Favaro et al., 2018; Han et al., 2017; Huang et al., 2015). In L2/3 of the V1 of juvenile wild-type (WT) mice, AMPAR-silent synapses are abundant ( $50 \%)$ at the eye opening (Funahashi et al., 2013; Rumpel et al., 2004); however, their numbers decline rapidly in an experience-dependent manner towards end of the CP for ODP (Favaro et al., 2018; Han et al., 2017; Huang et al., 2015). This process is promoted by postsynaptic density (PSD) protein-95 (Béïque et al., 2006; Schlüter et al., 2006), which is closely 
involved in trafficking and stabilizing AMPARs at the post-synaptic membrane of the synapse (Elias et al., 2006). In knockout (KO) mice, the absence of PSD-95 results in persistently elevated AMPAR-silent synapse fraction throughout adulthood (Favaro et al., 2018; Huang et al., 2015), while reducing PSD-95 expression via knockdown (KD) at full maturity reestablishes the elevated AMPAR-silent synapses cell autonomously (Huang et al., 2015). When tested for ODP at maturity, after a brief MD, both PSD-95 KO and KD mice show robust $O D$ shifts that are mechanistically similar to that of juvenile mice, suggesting that PSD-95 is necessary for CP closure. Moreover, OD shifts after MD are more transient in adult PSD-95 KO mice, and recover to normal faster (in 2 days versus 4 days in WT) when the deprived eye is reopened (Huang et al., 2015). These findings open a new avenue to further investigate the structural correlates of experience-dependent plastic changes in the adult brain of PSD-95 deficient mice, which is still as malleable by experience as the juvenile brain.

Given that the CP for ODP in PSD-95 deficient mice remains open into adulthood, we hypothesized that morphological signatures of CP plasticity are likely to be present in adult PSD-95 KO and KD mice. To test our hypothesis we have performed 2-photon imaging in the visual cortex of awake PSD-95-deficient and control mice with layer 2/3-specific GFP expression. We tracked the same spines longitudinally during consecutive 4-day periods of normal vision, monocular deprivation, and reopening of the deprived eye. In support of our hypothesis, we found that a brief MD leads to robust perturbation of spine dynamics in adult PSD-95-deficient mice, similar to what has been reported previously in juvenile mice (Sun et al., 2019; Zhou et al., 2017). 


\subsection{Methods}

\section{Experimental Animals.}

All experimental procedures were approved by the local government (Niedersächsisches Landesamt für Verbraucherschutz und Lebensmittelsicherheit, registration number 33.942502-04-15/1881). Heterozygote PSD-95 female mice with a mixed 129SV/C57BL/6J background (Abbas et al., 2009; Yao et al., 2004), bred at in-house animal facility, were mated with heterozygote males of the same line to get embryonic day (E) 15.5 PSD-95 WT and $\mathrm{KO}$ embryos for in utero electroporation. For generating layer 2/3-specific PSD-95 knockdown mice, C57BL/6J mice with timed pregnancies were obtained from the mouse colony of the central animal facility of the University Medical Center Göttingen for. All mice were housed in groups of $<5$ in standard cages $(26 \times 20 \times 14 \mathrm{~cm})$ with a 12-h light/dark cycle with food and water ad libitum. Mice were separated after the cranial window implantations and housed single until the end of experiments. Both sexes of animals were included in the study.

\section{In utero Electroporation.}

In utero electroporation was performed as previously described (Saito, 2006; Saito and Nakatsuji, 2001). Pregnant mice were anesthetized with isoflurane (3\% for induction and $1.5 \%$ during surgery), in $\mathrm{O}_{2}(0.8 \mathrm{~L} / \mathrm{min})$ and received injections of buprenorphine $(0.1 \mathrm{mg} / \mathrm{kg})$ and carprofen $(5 \mathrm{mg} / \mathrm{kg})$. Using a sterile surgical technique, uterine horns were exposed through a midline incision made in the abdominal wall. Left ventricle of E15.5 mouse embryos were injected with $\sim 0.5 \mu \mathrm{L}$ Tris-EDTA solution containing $2 \mu \mathrm{g} / \mu \mathrm{L}$ exdotoxin-free plasmid DNA and $0.1 \%$ Fast Green through a pulled glass micropipette using pedalcontrolled pressure injector. PSD-95 WT and KO embryos were injected a plasmid expressing CAG-driven eGFP (eGFP.CAG). For PSD-95 knockdown, another plasmid expressing shRNA against PSD-95 with a CAG-driven GFP tag (sh95.eGFP.CAG) or the control (eGFP.CAG) plasmid was injected to WT C57BL/6J embryos. Injections of lower volumes, but high concentration $(2 \mu \mathrm{g} / \mu \mathrm{L})$ of DNA solution was critical to achieving sparse expression. $5 \mathrm{~mm}$ tweezer type round platinum electrodes (NepaGene, CUY650P5) were positioned to target the dorsolateral wall of the left hemisphere and deliver a series of 5 square-wave current pulses ( $35 \mathrm{~V}, 50$-ms duration, 950-ms interval) generated by the pulse generator (Nepa21 electroporator, NepaGene). The abdominal wall was sutured shut after returning the embryos were into the mother's womb, and the dam was allowed to recover in a cage kept on a heating pad. 


\section{Chronic Cranial Window Implantation.}

For the repeated in-vivo imaging of dendrites, we implanted a cranial window over the left visual cortex of in-utero-electroporated mice ( P45) under 3-component general anesthesia (fentanyl $0.075 \mathrm{mg} / \mathrm{kg}$, midazolam $7.5 \mathrm{mg} / \mathrm{kg}$, medetomidine $0.75 \mathrm{mg} / \mathrm{kg}$, i.p.) as described before (Holtmaat et al., 2009; Joachimsthaler et al., 2015; Rose et al., 2016). Briefly, following a scalp incision and fascia cleaning, circular craniotomy was performed over the binocular visual cortex using steel drills ( $3 \mathrm{~mm}$ lateral to midline, $1 \mathrm{~mm}$ anterior to lambda). Drilled bone was removed taking extensive care not to damage the dura, and the exposed surface was kept moist by applying $0.9 \%$ saline gel foam. A 4-mm round coverslip was then gently placed onto the brain tissue and secured to the surrounding bone using light curing cement (Tetric EvoFlow). A custom made titanium ring $(0.7 \mathrm{~g}, 14 \mathrm{~mm})$ for the head restraining was subsequently glued to the cement around the cover slip (Hefendehl et al., 2012), and skin was sutured to enclose the ring. Anesthesia was terminated by an antidote (naloxon $1.20 \mathrm{mg} / \mathrm{kg}$, flumazenil $0.50 \mathrm{mg} / \mathrm{kg}$, atipamezol $2.50 \mathrm{mg} / \mathrm{kg}$, s.c.), and general analgesia was provided by carprofen injection $(5 \mathrm{mg} / \mathrm{kg})$ postoperatively. Animals were closely monitored for at least five days for recovery before starting the consequent imaging and habituation steps.

\section{Optical Imaging of Intrinsic Signals.}

Visual cortical responses were recorded through the implanted cranial window using the optical imaging of intrinsic signals (Cang et al., 2005a; Kalatsky and Stryker, 2003), as described before (Greifzu et al., 2016; Greifzu et al., 2014). Mice were anesthetized with $0.6-0.8 \%$ isoflurane in oxygen, and the body temperature was maintained at $37^{\circ}$ using a feedback-controlled heating pad. A temporally periodic stimulus was continuously presented to the animal, and the cortical response at the stimulus frequency was extracted by Fourier analysis. Optical images of intrinsic cortical signals were obtained using $135 \times$ 50-mm tandem lens configuration (Nikon), and a Dalsa 1M30 CCD camera. The surface vascular pattern and intrinsic signal images were visualized with illumination wavelengths set by a green $(550 \pm 10 \mathrm{~nm})$ or red $(610 \pm 10 \mathrm{~nm})$ interference filter, respectively. After acquisition of a surface image, the camera was focused $600 \mu \mathrm{m}$ below the blood vessels. An additional red filter was interposed between the brain and the CCD camera. Frames were acquired at a rate of $30 \mathrm{~Hz}$ temporally binned to $7.5 \mathrm{~Hz}$ and stored as $512 \times 512$ pixel images after spatial binning of the camera image. A drifting horizontal bar restricted to the binocular visual field of the left primary visual cortex $\left(20^{\circ}\right.$ wide, $-5^{\circ}$ to $+15^{\circ}$ azimuth $)$ was presented to the both eyes at a distance of $25 \mathrm{~cm}$ on a high refresh rate monitor. Two consecutive activity maps obtained from each five minute imaging sessions with drifting 
bars moving in either $90^{\circ}$ and $270^{\circ}$ direction were averaged to get the retinotopic map of binocular $\mathrm{V} 1$. On average, animals were anesthetized for less than 30 minutes.

\section{Two-Photon Imaging}

Awake imaging was performed using a custom-made two-photon microscope. A modelocked Ti:Sapphire laser (tunable Chameleon laser, Coherent) was tuned to $920 \mathrm{~nm}$, and the maximum laser power reaching the tissue was below $40 \mathrm{~mW}$. Emission light was collected by a 40x water-immersion objective (NA 0.8; Olympus), filtered by emission filter (525/20; BrightLine HC) and captured by a photomultiplier tube (Hamamatsu H10770PA). Scanning and image acquisition was controlled by Scanlmage software (Pologruto et al., 2003). On the following day of intrinsic signal imaging, mice were shortly (> $15 \mathrm{~min}$ ) screened using two-photon microscope while anesthetized to confirm GFP expression in binocular visual cortex ( $0.6 \%$ isoflurane in oxygen). Animals with bright labeling of dendrites in binocular V1 were proceeded to the habituation steps for subsequent awake repeated imaging sessions. A restrainer box described before was used for head-fixing the animals under two-photon microscope (Joachimsthaler et al., 2015; Schwarz et al., 2010). Animals were habituated to the box and were gradually trained to be restrained from 30 seconds up to 1 hour during 2 - 3 weeks of daily training. A scoring criteria was used to rate the behavior and the distress signs of mice for ensuring stress-free habituation to head restraining. Blood vessel pattern and $X Y$ coordinates was used to re-localize the imaged dendrites within a few micrometers precision each imaging session. Per animal, 15 - $40 \mu \mathrm{M}$ Z-stacks $(0.5 \mu \mathrm{m}$ steps) of three to five regions within binocular V1 were acquired at $1024 \times 1024$ (0.05 $\mu \mathrm{m} / \mathrm{pixel}$ ) resolution with $2.5 \mathrm{x}$ digital zoom at a maximum depth of $\sim 80 \mu \mathrm{M}$ from pia. Each $z$ plane was scanned four to seven times in order to perform post-hoc correction of the motion artefacts caused by small movements and breathing.

\section{Monocular deprivation and reopening.}

Immediately after the last baseline imaging session (d4), right eye was deprived of vision for the next 4 days as described previously (Gordon and Stryker, 1996). Mice were anesthetized with $2 \%$ isoflurane in $1: 1 \mathrm{O}_{2}: \mathrm{N}_{2} \mathrm{O}(<15$ minutes in total), and analgesia was provided by carprofen (sc, $5 \mathrm{mg} / \mathrm{kg}$ ). Lid margins were trimmed, and an antibiotic gel (IsoptoMax, Novartis) was applied. The eye was closed with two mattress sutures (Ethicon, 7-0). Mice were checked twice daily to make sure that the eyes remained closed. Deprived eye was then reopened on d8 immediately after the imaging session, under brief ( $<5 \mathrm{~min}$ ) $2 \%$ isoflurane $\left(1: 1 \mathrm{O}_{2}: \mathrm{N}_{2} \mathrm{O}\right)$ anesthesia and carprofen $(\mathrm{sc}, 5 \mathrm{mg} / \mathrm{kg})$ analgesia, and an antibiotic 
gel was applied. Reopened eye was checked twice daily and received additional antibiotic gel treatments to make sure that it remained open and uninfected.

\section{Data Analysis and Statistics.}

Stacks were motion corrected using a custom-written MATLAB script that performs an initial rigid $x-y$ transformation followed by a non-rigid frame warping based on Lukas Kanade method (Greenberg and Kerr, 2009). Resulting images were then deconvoluted using AutoQuant X (Media Cybernetics), and contrast adjusted with Fiji using Enhance Contrast function to improve the visibility of spines (Schindelin et al., 2012). Spine counting was performed blind to the genotype and the imaging session. A custom-written MATLAB script (Joachimsthaler et al., 2015) was used to align dendrites in several stacks and thus made following spines across imaging days easier. $>20 \mu \mathrm{m}$ segments of dendrites lying in the horizontal plane were included in the analysis. We defined spines as all the protrusions that appeared on at least 2 consecutive $z$ planes and were longer than $0.4 \mu \mathrm{m}$. Filopodia like structures were rarely encountered in either genotype and thus are unlikely to have made any difference in our conclusions. Spine elimination and formation ratios were quantified as (number of lost or gained spines) / (number of pre-existing spines + number of resulting spines).

Due to hierarchical and longitudinal design of our study we have opted for linear and generalized linear mixed-effects models (LME and GLME, respectively) in our statistical analyses of spine elimination and formation ratios, allowing us to also account for random effects such as animal and dendrite ID. Normality of data was checked by Shapiro-Wilk test, and only normally distributed data were presented as mean \pm SEM. As the histograms of spine elimination and formation ratios were right skewed, we have used GLME to fit a gamma distribution with log link to analyze the spine elimination and formation ratios. To further confirm our GLME analysis we have normalized the data by calculating MD-induced change in spine elimination or formation ratios ( $\Delta_{E}$ and $\Delta_{F}$, respectively) as $R_{M D}-R_{N V}$ (see Results), and performed 2-way ANOVA or LME analysis. Exact $F$ values for experimental variables such as genotype $\left(F_{g}\right)$, visual experience $\left(F_{v}\right)$, MD $\left(F_{m d}\right)$, time $\left(F_{t}\right)$, and 2- or 3way interactions between variables are reported where appropriate alongside Bonferroni adjusted $p$ values for planned pairwise contrasts. Statistical analyses were done using the IBM SPSS and Prism 8.4.0. 


\subsection{Results}

\section{Repeated in vivo imaging of dendritic spines in awake PSD-95 WT and KO mice.}

To image the dendritic spine dynamics of layer $(L) 2 / 3$ pyramidal neurons in $V 1$, we performed in-utero electroporation at E15.5 to achieve layer-specific expression of eGFP in both PSD-95 WT and KO mice (Fig. 1A). Post-hoc inspection of coronal brain slices from adult mice (>P100) electroporated with either of the constructs have confirmed that the expression was exclusive to L2/3 (Fig. 1B, F). At around postnatal day (P) 45, a cranial window was implanted by replacing a $4 \mathrm{~mm}$ diameter round piece of cranium over the binocular V1 with a glass coverslip (Fig. 1C). 5-10 days (d) after the window implantation, we have identified the binocular V1 using intrinsic signal optical imaging (Fig. 1D) (Kalatsky and Stryker, 2003). Mice with bright and sparse labeling of dendrites with clearly identifiable dendritic spines (Fig. 1E, F) were proceeded to a stepwise habituation procedure (see Methods) for awake head restraining under the two-photon microscope up to 1 hour. Experimental protocol consisted of three phases that spanned $12 \mathrm{~d}$ in total: normal vision (NV: d0-d4), monocular deprivation (MD: d4-d8) of the contralateral eye, and recovery after reopening (RO: d8-d12) the deprived eye (Fig. 1A). Trained awake mice were imaged repeatedly with 1- and 4-day (d) intervals during NV and MD, and with 2- and 4-d intervals during RO. We have opted for 2-d intervals during RO to match the timeline used in our previous study (Huang et al., 2015).

\section{4-d MD reduced the spine density in apical dendrites of L2/3 PNs in PSD-95 KO mice.}

In adult PSD-95 KO mice, 4-d MD causes a reduction of the deprived eye responses in the binocular V1 (Huang et al., 2015), similar to CP mice (Gordon and Stryker, 1996). However, structural changes underlying the reduced deprived eye responses in adult PSD-95 KO have not been investigated. We have repeatedly imaged 40 dendrites $(\sim 948 \mu \mathrm{m})$ in binocular V1 of six PSD-95 WT mice (P77-79; 78), and 36 dendrites ( 936 $\mu \mathrm{m})$ in six KO mice (P64-79; $~ 73$ ) with 4-d intervals during NV and MD (Fig. 1A). A majority of those dendrites (25/4, WT; 20/3, KO) were also imaged with 1-d intervals, on the first days NV of MD phases (Fig. 1A). Spine densities on d0 were not significantly different between WT (0.843 \pm 0.022 spine $/ \mu \mathrm{m})$ and $\mathrm{KO}$ mice $(0.767 \pm 0.030 \mathrm{spine} / \mu \mathrm{m}$; linear mixed-effects model $(\mathrm{LME}): F_{\mathrm{g}}=6.756, F_{\mathrm{t}}=6.022, F_{\mathrm{gxt}}=2.110 ; \mathrm{d0}$ : WT vs. KO, $\left.p=0.217\right)(\mathrm{Fig} 2 A)$. We then normalized the spine densities in all sessions to the respective baseline (d0) values, and compared the magnitude of spine density changes between and within genotypes during 12 days of imaging (Fig 2B). Differences between WT and KO mice were not statistically 
significant on any of the imaging days (LME: $\left.F_{\mathrm{g}}=0.024, F_{\mathrm{t}}=12.80, F_{\mathrm{g} \times \mathrm{t}}=1.712\right)$. However, intragroup comparison of the spine densities to $\mathrm{d} 0$ revealed a significant reduction of spine density in KOs after $4-d \mathrm{MD}(-6.36 \pm 3.38 \%, p=0.037)$, and on $2-\mathrm{d} \mathrm{RO}(-8.07 \pm 3.91 \%, p$ $=0.008)$, suggesting that MD have unbalanced the spine dynamics in $\mathrm{KO}$ mice.

A
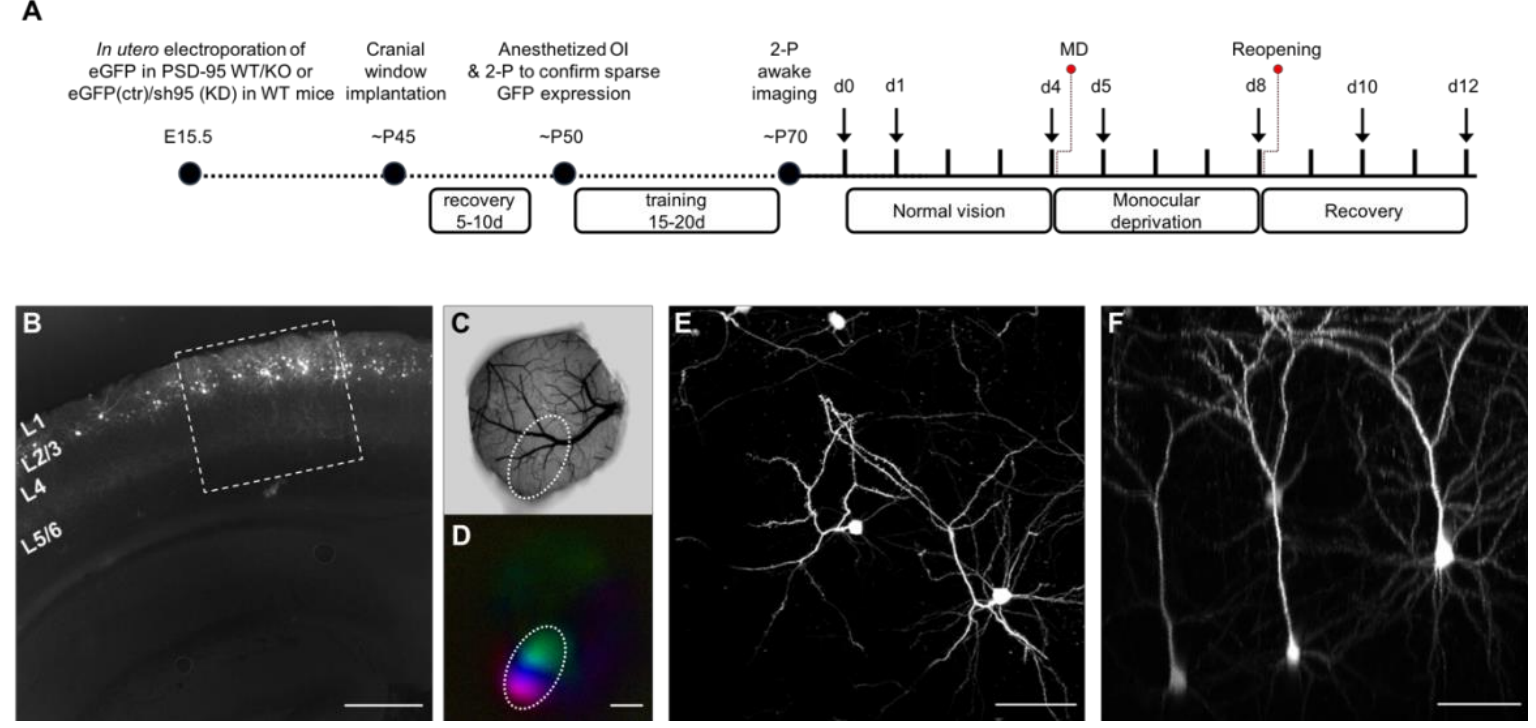

Figure 1. Experimental design and labeling of dendritic spines in L2/3 PNs. (A) Timeline, plasmids expressing eGFP (sh95.eGFP in knockdown mice) were delivered into the left ventricle of E15.5 embryos. After craniotomy ( P45), optical imaging of intrinsic signals was used to locate the binocular V1. Mice ( P50) were then screened for sparse neuronal labeling in the binocular V1 and proceeded to a 15-20d habituation procedure for up to 1 hour head-restraining. MD was performed on day 4 (d4) by suturing the contralateral eyelid and was reopened on d8. Awake two-photon Imaging was done on days indicated by the black arrows. (B) Coronal slice of an electroporated brain with L2/3-specific GFP expression (scale bar, $400 \mu \mathrm{m}$ ). (C) Cortical blood vessel pattern imaged through the cranial window with an outline of binocular V1 (dashed ellipse), identified by (D) retinotopic mapping of mouse binocular V1 using optical imaging (scale bar, 1mm). (E) Lowmagnification top view of an imaged region with sparsely eGFP-labeled L2/3 PNs (scale bar: $50 \mu \mathrm{m})(\mathbf{F})$ Side-view of the z-stack in (E) (scale bars: $50 \mu \mathrm{m}$ ). 

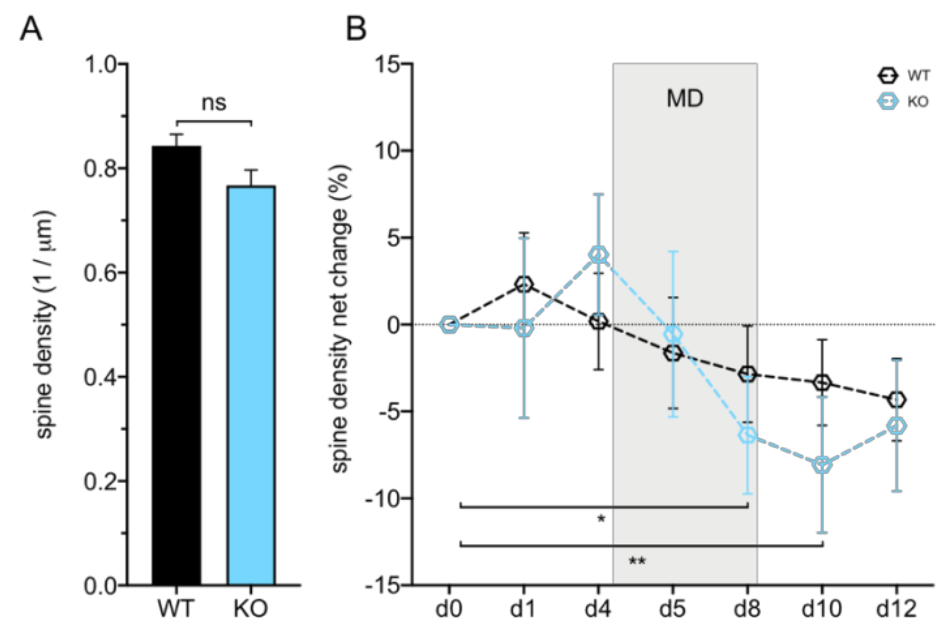

Figure 2. Repeated spine density measurements in PSD-95 WT and KO L2/3 apical dendrites during normal vision (NV), monocular deprivation (MD), and binocular recovery (RO). (A) Spine densities of apical dendrites in PSD-95 WT and KO mice on d0. (B) Same dendrites were repeatedly imaged with 1-d (4 WT/4 KO: 27/21 dendrites) and 4d (6 WT/6 KO: 40/36 dendrites) intervals during NV and MD periods. Imaging was continued 2-d (5 WT/5 KO: 27/21 dendrites) and 4-d (5 WT/5 KO: 40/36 dendrites) after reopening the deprived eye. Spine density was significantly reduced in PSD-95 KO mice during 4-d MD and the reduction persisted after $2 \mathrm{~d}$ of RO. ${ }^{*} p<0.05 ;{ }^{* *} p<0.01$

\section{4-d MD unbalances spine dynamics in PSD-95 KO mice towards more spine elimination and relatively less spine formation.}

To understand the cause of MD-dependent reduction in spine density in PSD-95 KO mice, we tracked the individual spines on PSD-95 WT (Fig. 3A) and KO (Fig. 3B) dendrites during $1-d$ and 4-d intervals of normal and monocular vision. During normal vision spine elimination ratios did not differ significantly between the genotypes for $1-d(p=0.748)$ and $4-d(p=$ 0.326 ) periods (Fig. $3 C, D$ ) (mean $\pm \mathrm{SD}$; generalized linear mixed-effects model (GLME): $\left.F_{\mathrm{g}}=1.851, F_{\mathrm{md}}=7.792, F_{\mathrm{t}}=2.486, F_{\mathrm{g} \times \mathrm{md}}=6.005\right)$. $1-\mathrm{d} \mathrm{MD}$ increased the spine elimination ratio in $\mathrm{KO}$ mice (Fig. $3 \mathrm{C}$ ) only moderately from $0.047 \pm 0.041$ during 1 -d $\mathrm{NV}$ to $0.064 \pm$ $0.040(p=0.204)$. However, 4-d MD led to a drastic increase in spine elimination (Fig. 3B, $D)$ from $0.039 \pm 0.033$ during $4-\mathrm{d} N V$ to $0.088 \pm 0.068 \quad(p<0.001)$, which was also significantly higher than the spine elimination ratios of WT controls after 4-d MD (0.032 \pm 0.029, $p=0.017$ ) (Fig. 3D). Consequently, the MD-induced net change (Fig. 3E) in spine elimination ratios $\left(\Delta_{E}\right)$ for 1-d intervals was only slightly different in KOs $(0.017 \pm 0.112)$ compared to WT mice $(-0.004 \pm 0.011)\left(\mathrm{LME}: F_{\mathrm{g}}=5.964, F_{\mathrm{t}}=4.303, F_{\mathrm{g} \times \mathrm{t}}=1.987 ; p=0.738\right)$. However, $\Delta_{E}$ for 4-day intervals was clearly higher in KOs $(0.049 \pm 0.013)$ compared to WT $(0.004 \pm 0.008, p=0.004)$ (Fig. $3 E)$. 


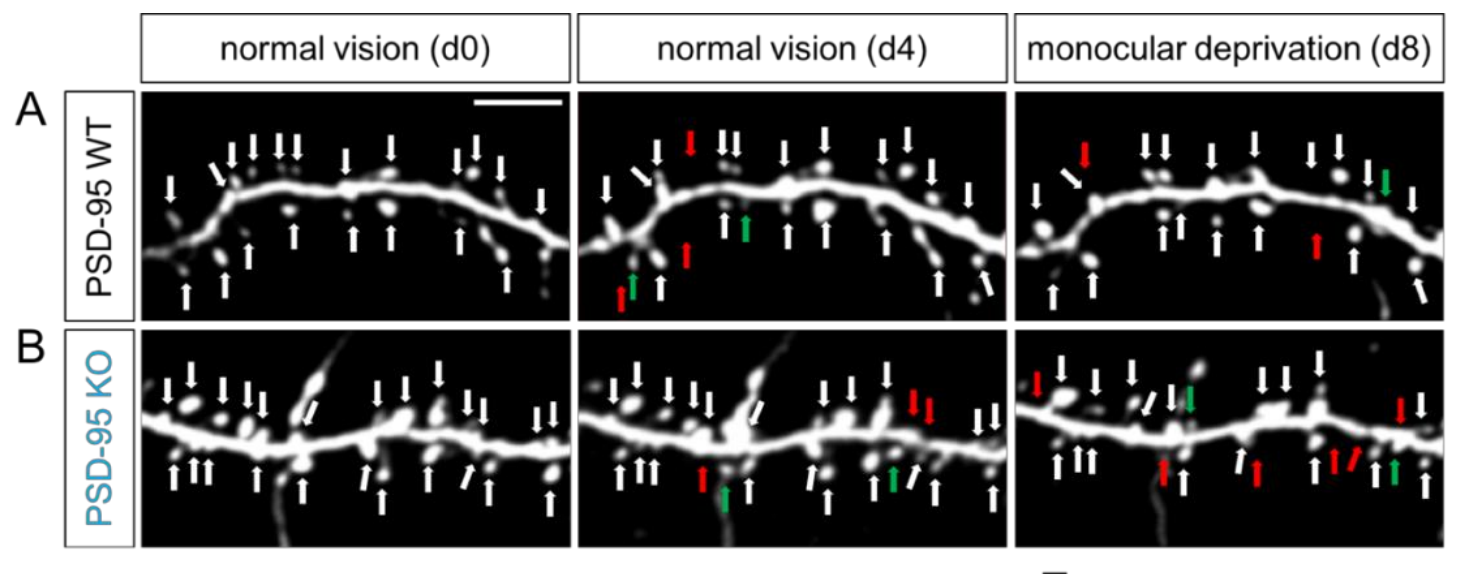

C

D
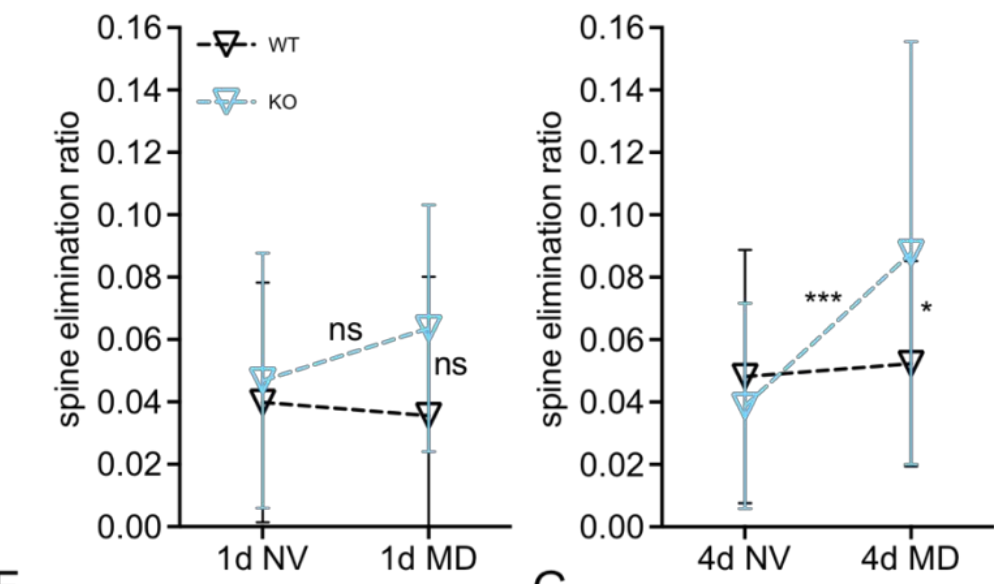

E

$\mathrm{F}$

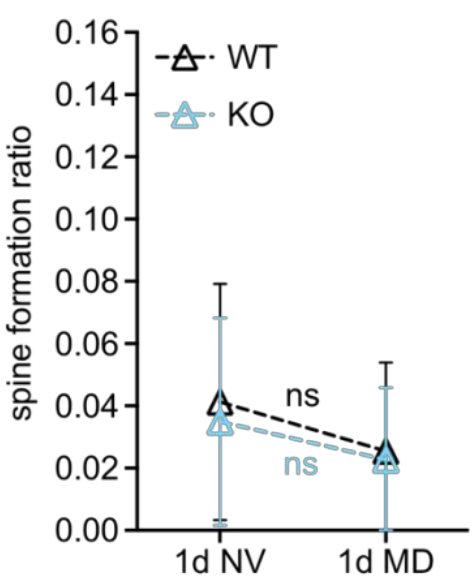

G

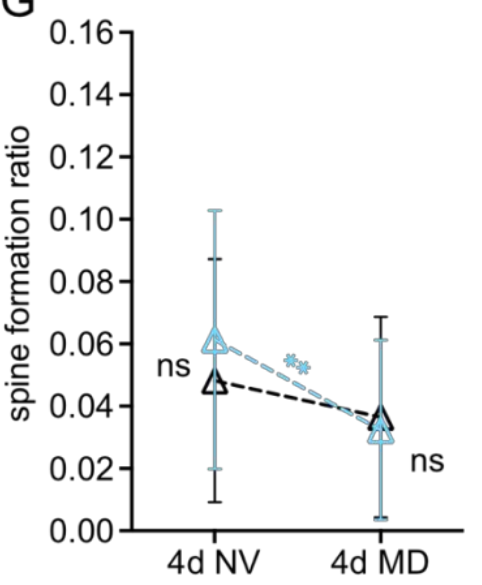

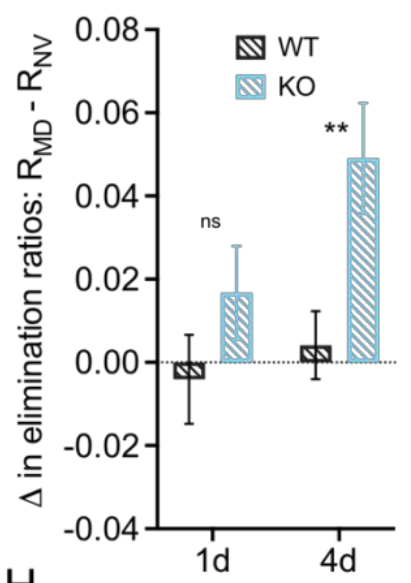

$\mathrm{H}$

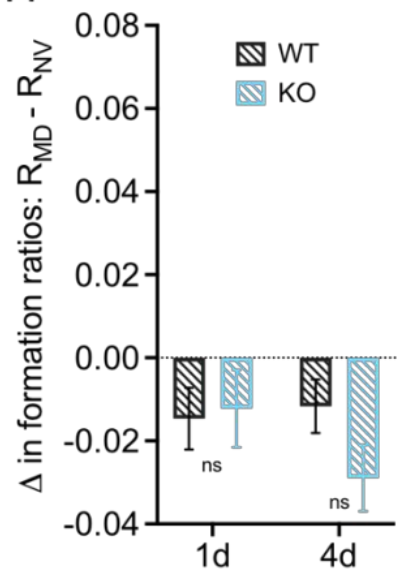

Fig 3. Experience-dependent spine dynamics in apical dendrites of $L 2 / 3$ PNs. (A) Representative examples of dendrites from PSD-95 WT and (B) KO mice on d0 (left), d4 (middle), and d8 (right): red arrows mark eliminated, green arrows mark formed spines during subsequent 4-d intervals (scale bar: $5 \mu \mathrm{m}$ ). (C,D) Mean ( \pm SD) spine elimination and $(\mathbf{F}, \mathbf{G})$ spine formation ratios during $(\mathbf{C}, \mathbf{F})$ 1-d and $(\mathbf{D}, \mathbf{G})$ 4-d intervals, calculated as $R=$ $N_{\text {elmn }}$ or $N_{\text {form }} /\left(N_{\text {initial }}+N_{\text {final }}\right)$. (E) MD-induced change $\Delta=\mathrm{R}_{M D}-R_{N V}$ in spine elimination and (H) formation ratios (mean \pm SEM). ${ }^{*} p<0.05 ;{ }^{* *} p<0.01 ;{ }^{* * *} p<0.001$. 
Spine formation ratios were also comparable between WT and KOs during 1-d $(p=0.438)$ and 4-d $(p=0.194) \mathrm{NV}$ periods (Fig. 3F,G) (GLME: $F_{\mathrm{g}}=0.052, F_{\mathrm{md}}=12.944, F_{\mathrm{t}}=9.978$, $\left.F_{\mathrm{g} \times \mathrm{md}}=0.150\right)$. In PSD-95 KO mice, 1-d MD did not affect spine formation rate (Fig. 3F) (mean \pm SD; NV $0.032 \pm 0.033$ vs. MD $0.023 \pm 0.023, p=0.307$ ), but it was significantly reduced after 4-d MD (Fig. $3 G$ ) (NV $0.061 \pm 0.042$ vs. MD $0.032 \pm 0.029, p=0.001$ ) nevertheless, was not significantly different from WT controls (Fig. 3F) (4-d MD WT: 0.037 $\pm 0.032, p=0.596$ ). Similarly, $\Delta_{\mathrm{F}}$ was comparable between WT and KO for 1-d (Fig. $3 H$ ) $\left(\right.$ LME: $F_{\mathrm{g}}=0.375, F_{\mathrm{t}}=1.498, F_{\mathrm{gxt}}=3.783$; mean \pm SEM: WT $-0.015 \pm 0.007$ vs. KO -0.012 $\pm 0.009, p>0.9$ ) or $4-d$ intervals (Fig. $3 H$ ) (WT $-0.012 \pm 0.006$ vs KO $-0.029 \pm 0.008, p=$ 0.161). In summary, MD-induced decrease in spine density in PSD-95 KO dendrites is explained by a stronger increase in elimination and only a relative decrease in spine formation.

Temporary spines in PSD-95 KO mice are particularly more likely to be eliminated after MD

Dendritic spines with lifetime of $>4$ days are typically classified as persistent, while temporary spines may survive only a few days, or even hours (Berry and Nedivi, 2017). MD-induced increase in spine elimination in PSD-95 KOs led us hypothesize that in KOs, a smaller percentage of spines present on d0 would still persist after 4-d MD. Interestingly, we did not find any significant differences between genotypes in the percentages of persistent spine populations (Fig. 4A) during 4-d NV (mean \pm SD, d4: WT 90.7\% \pm 7.51 vs. KO $92.3 \% \pm 6.28$ ) or after $4-d$ MD (d8: WT $83.6 \% \pm 9.40$ vs. KO $82.1 \% \pm 10.2$, KolmogorovSmirnov test, $p=0.689$ ). This result suggested that in PSD-95 KO mice MD-induced structural reorganization has preferentially targeted temporary spine populations. We have then grouped WT and KO dendrites according to the fate of spines newly formed during NV (Fig 4B; $0.00=$ all new spines were stabilized, $1.00=$ all new spines were lost after MD). Interestingly, only in $18.2 \%$ (in contrast to $52.5 \%$ in WT) of KO dendrites all of the newly gained spines were stabilized (not lost during MD), and in $30.3 \%$ of KO dendrites (vs. $9.1 \%$ in WT) all newly gained spines were eliminated during MD (Fig. 4B). Comparison of cumulative distribution of spine stability ratios between WT and KO yielded a significant difference (Kolmogorov-Smirnov test, $p=0.026$ ), showing a shift towards less spine stability in PSD-95 KO mice. 
A

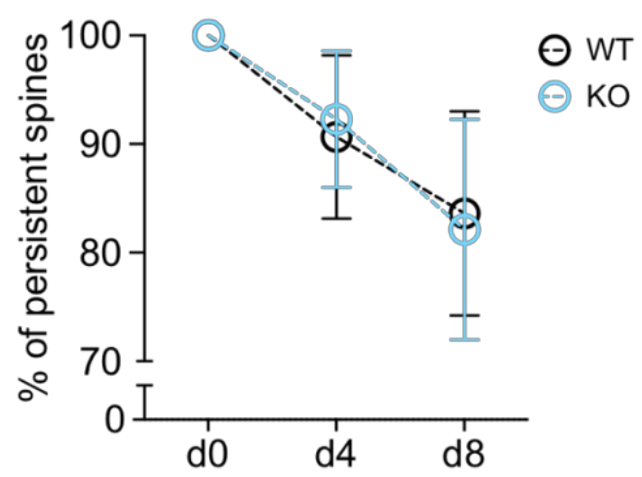

B

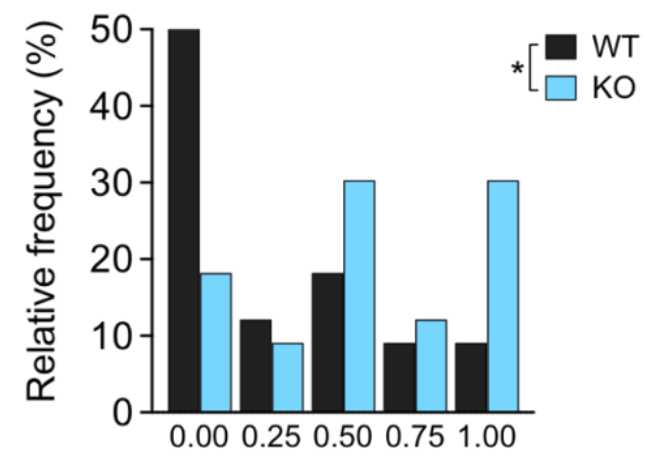

Fig 4. MD-induced changes in persistent and newly formed spine populations. (A) Percentage of persistent spines (mean \pm SD) present from d0 throughout $N V$ and MD periods. (B) Histogram depicts relative frequency of PSD-95 WT and KO dendrites binned according to the fractions of newly formed spines that were eliminated during 4-d MD $(0.00$ $=$ none eliminated; $1.00=$ all new spines eliminated during MD). ${ }^{*} p<0.05$

\section{Spine dynamics partially normalize after 4 days of binocular recovery}

Fast recovery of deprived-eye responses after reopening the MD is another characteristic feature of CP plasticity (Espinosa and Stryker, 2012). In adult PSD-95 KO mice, neuronal responses return to normal within $2 \mathrm{~d}$ of reopening the MD, while in age-matched WT controls this takes $4 \mathrm{~d}$ (Huang et al., 2015). It was, however, untested whether faster recovery from MD in PSD-95 KO mice is mediated by the structural reorganization of synapses. To answer this question, we have compared the spine dynamics in PSD-95 KO $(n=6)$ mice to WT $(n=6)$ controls 2 d (WT/KO: 33/31dendrites) and 4 d (33/30) after reopening the MD eye (Fig. 5A, B). Unexpectedly, $2 \mathrm{~d}$ after reopening, we did not find any significant differences in spine elimination (Fig. 5C) (mean \pm SD; GLME: $F_{\mathrm{g}}=0.306, F_{\mathrm{t}}=$ 3.052; WT $0.058 \pm 0.030$ vs. KO $0.056 \pm 0.041, p=0.583$ ) or formation ratios (Fig. $5 D$ ) (mean \pm SD; GLME: $F_{\mathrm{g}}=0.933, F_{\mathrm{t}}=5.202$; WT; $0.042 \pm 0.040$ vs. KO $0.044 \pm 0.035, p=$ 0.978 ) between WT and KO mice. $4 \mathrm{~d}$ after reopening, spine elimination (Fig. 5C) (WT 0.070 \pm 0.037 vs. KO $0.065 \pm 0.046, p=0.701$ ) and formation rates were still comparable between genotypes (Fig. 5D) (WT $0.049 \pm 0.033$ vs. KO $0.068 \pm 0.041, p=0.093$ ). 

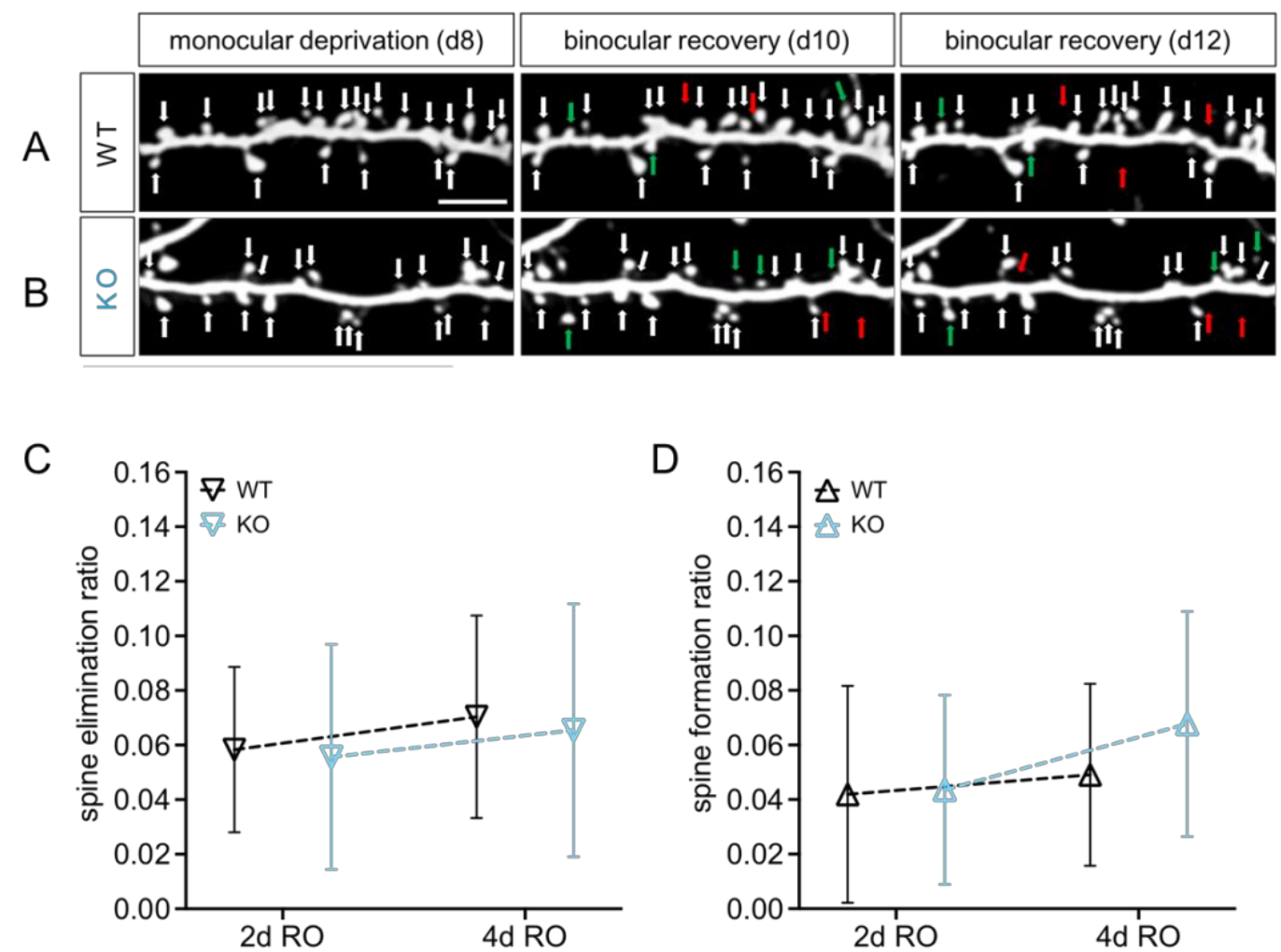

E

F
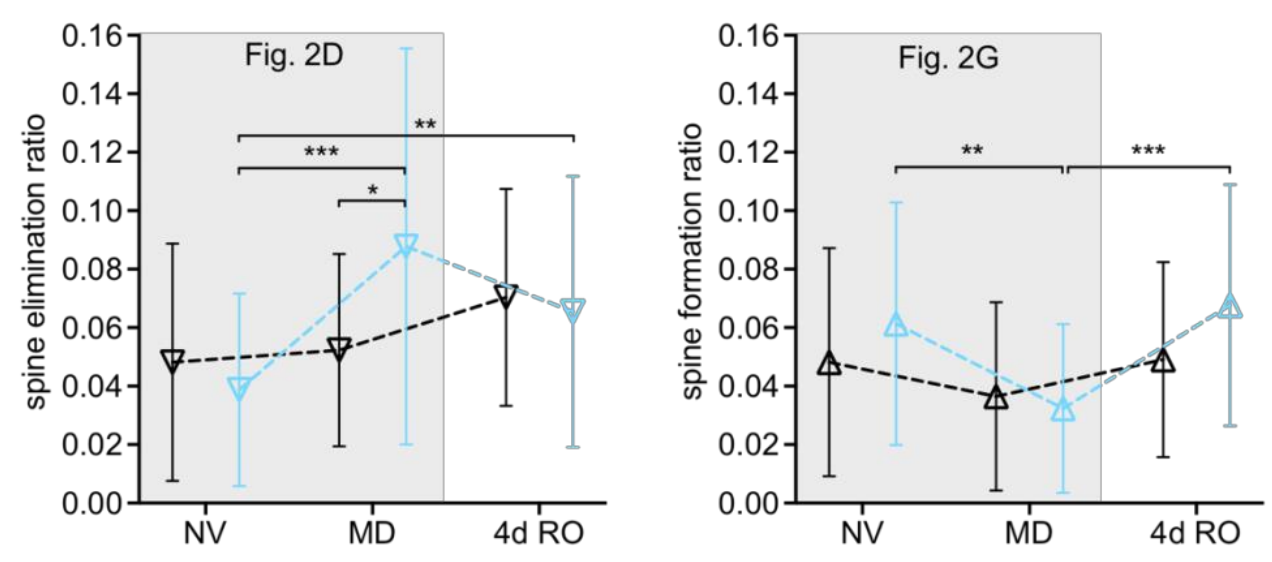

Fig. 5 Spine dynamics after reopening the previously deprived eye. A subset of dendrites described in Fig. 3 were repeatedly imaged 2 d (6WT/6 KO: 33/31 dendrites) and $4 \mathrm{~d}$ ( $6 \mathrm{WT} / 6 \mathrm{KO}: 33 / 30$ dendrites) after reopening the MD eye. (A) Representative examples of dendrites from PSD-95 WT and (B) PSD-95 KO mice on d8 (left), d10 (middle), and d12 (right) Arrows mark formed (green) and eliminated (red) spines in respect to d8 (scale bar: $5 \mu \mathrm{m})$. (C) Mean ( \pm SD) spine elimination and (D) spine formation ratios during 2-d and 4-d intervals after reopening $(E)$ Comparison of mean $( \pm S D)$ spine elimination and $(F)$ formation ratios during 4-d reopening to NV and MD periods (grey shaded area, data replotted from Fig. $3 D$ and $3 G$ for comparison. ${ }^{*} p<0.05 ;{ }^{* *} p<0.01 ;{ }^{* * *} p<0.001$. 
We then tested whether spine dynamics 4-d after reopening were similar to 4-d NV or MD periods within genotypes (Fig. 5E, F). In WT controls, during 4-d RO spine elimination remained comparable (Fig. $5 E$ ) (GLME: $\left.F_{g}=0.590, F_{v}=8.494, F_{g^{*} v}=5.363\right)$ to 4-d NV $(p=$ $0.075)$ or 4-d MD ( $p=0.126$ ) phases. In PSD-95 KO mice, we found that during 4-d RO, spine elimination ratios $(0.065 \pm 0.046)$ were only slightly lower than 4 -d MD $(0.088 \pm 0.068$, $p=0.075)$, but still significantly elevated compared to $4-d$ NV (Fig. $5 E)(0.039 \pm 0.032, p=$ $0.009)$.

Spine formation ratios were not perturbed in WT mice during 4-d RO (Fig. 5F) (GLME: $F_{\mathrm{g}}=$ 1.954, $\left.F_{\mathrm{v}}=9.403, F_{g^{*}}=1.611\right)$, compared to $4-\mathrm{d} \mathrm{NV}(p=0.914)$ or $4-\mathrm{d}$ MD phases $(p=$ 0.268). In PSD-95 KO mice, formation ratios were similar to the baseline values during 4-d $\mathrm{RO}$ (Fig. 5F) (RO $0.068 \pm 0.035$ vs. NV $0.061 \pm 0.042, p=0.561)$, but significantly higher than MD (Fig. 4F; $0.032 \pm 0.029, p<0.001$ ) values. In summary, after 4-d RO, spine dynamics were similar between PSD-95 WT and KO mice, although increased spine elimination in PSD-95 KOs did not fully return to the baseline values. Comparison of spine dynamics after 2-d RO did not reveal any significant differences between the genotypes. These data suggest that in PSD-95 KO mice, other mechanisms (see Discussion) are likely to be involved in the prompt recovery of neural responses after reopening the MD eye.

\section{PSD-95 function in regulating spine stability is cell autonomous.}

It was previously shown that V1-specific silencing of PSD-95 expression is sufficient to preserve juvenile-like ODP in adult mice, similar to PSD-95 KO mice (Huang et al., 2015). Here we explored whether deletion of PSD-95 in a subset of L2/3 PNs in the binocular V1 would also make these neurons more susceptible to experience-dependent structural reorganizations, similar to $\mathrm{KO}$ neurons. Using the same imaging protocol as for the experiments with PSD-95 KO and WT mice, apical dendrites coming from L2/3 PNs expressing sh95 ( $n=19 / 3$ mice; $\sim 515 \mu \mathrm{m}$; P73-74) or GFP plasmid ( $n=21 / 4$ mice, $\sim 475$ $\mu \mathrm{m}$; P82-83) were repeatedly imaged with 1- and 4-d intervals during NV and MD (Fig. 6A, $B)$. Spine densities were similar on d0 between control $(0.843 \pm 0.032 \mathrm{spine} / \mu \mathrm{m})$ and sh95 groups (Fig. $6 C)\left(0.870 \pm 0.045\right.$; LME: $\left.F_{\mathrm{g}}=0.3520, F_{\mathrm{t}}=2.720, F_{\mathrm{g}^{*} \mathrm{t}}=1.493 ; p>0.9\right)$. After 4$\mathrm{d} M D$, in contrast to our previous findings in PSD-95 KO mice, the reduction in spine density in sh95 dendrites was non-significant (Fig. $6 C)\left(-2.044 \pm 5.270 \%\right.$; LME: $F_{\mathrm{g}}=2.540, F_{\mathrm{t}}=$ $\left.1.69, F_{\mathrm{g}^{* t}}<0.001 ; p>0.9\right)$. 

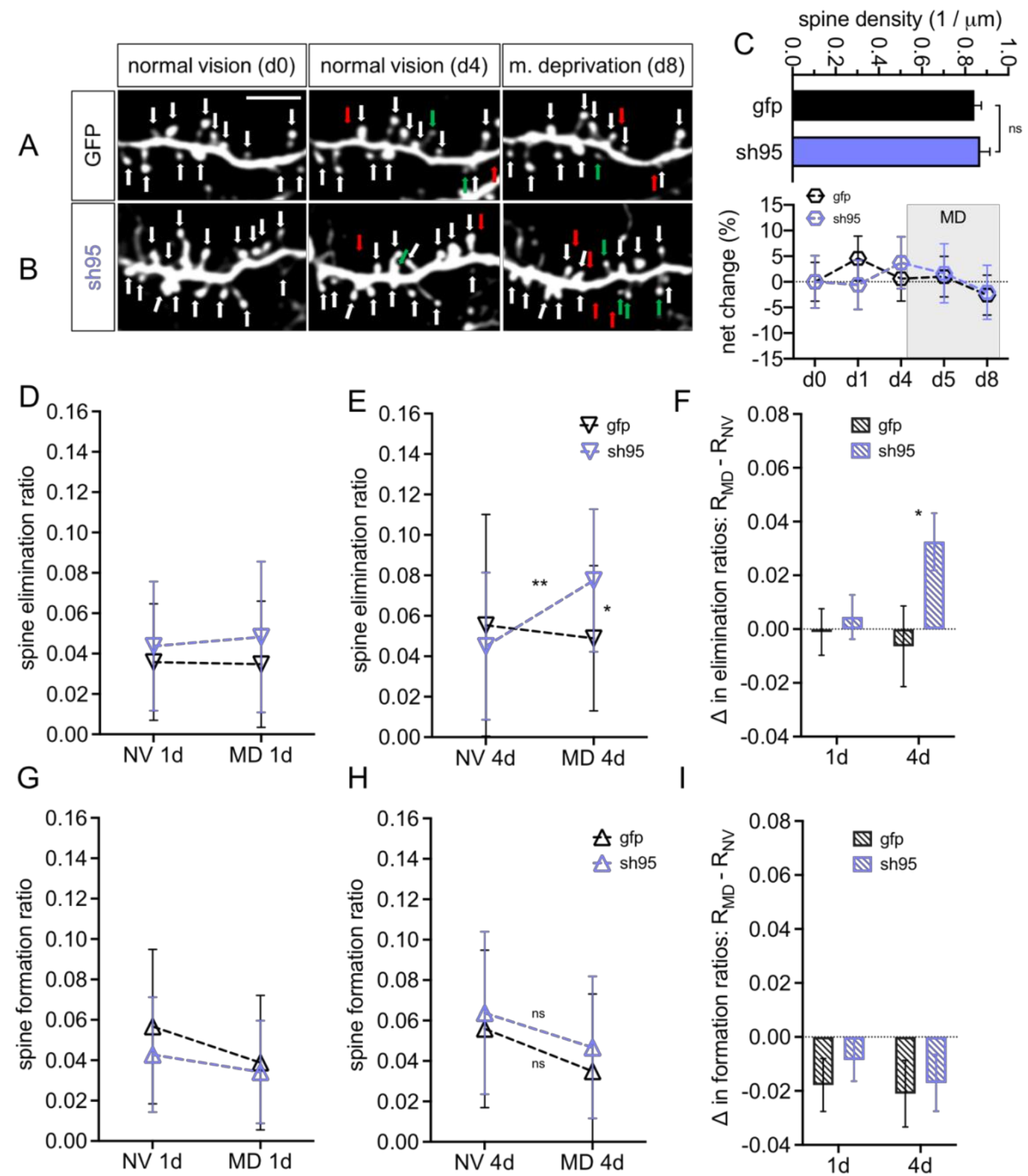

Fig 6. Experience-dependent spine dynamics in sh95 L2/3 PNs (A) Apical dendrites of L2/3 PNs electroporated expressing control (4 mice / $n=19)$ or $(B)$ sh95 plasmids (3 mice / $\mathrm{n}=21$ ) were repeatedly imaged with 1 - and 4-d intervals during NV and MD periods: red arrows mark eliminated, green arrows mark formed spines during 4-d intervals (scale bar: $5 \mu \mathrm{m})$. (C) Average spine densities ( \pm SEM) during NV and MD periods (grey shaded area). (D-E) Mean ( \pm SD) spine elimination and (G-H) spine formation ratios during (D and G) 1$\mathrm{d}$ and ( $\mathbf{E}$ and $\mathbf{H})$ 4-d intervals. (F) MD-induced change ( $\left.\Delta=\mathrm{R}_{\mathrm{MD}}-\mathrm{R}_{\mathrm{NV}}\right)$ in spine elimination and (I) formation ratios (mean \pm SEM). ${ }^{*} P<0.05$; ${ }^{* *} P<0.01$; ${ }^{* * *} P<0.001$. 
Spine elimination ratios were similar between GFP and sh95 during $1-\mathrm{d}(p=0.503)$ and 4d NV $(p=0.516$ ) periods (Fig. $6 D, E)$ (GLME: $F_{\mathrm{g}}=1.265, F_{\mathrm{md}}=1.409, F_{\mathrm{t}}=6.767$, $F_{g^{*} \mathrm{md}}=3.433$ ). As we have previously observed in PSD-95 WT and KO mice, 1-d MD did not lead to significant changes in spine elimination (Fig. 6D) (mean \pm SD; ctrl: 1-d NV $0.036 \pm$ 0.029 vs. $1-d$ MD $0.035 \pm 0.031, p=0.204$; sh95: $1-d$ NV $0.044 \pm 0.032$ vs. $1-d$ MD 0.048 $\pm 0.037, p=0.568)$. However, after $4-d \mathrm{MD}$, spine elimination ratios were significantly increased in sh95 dendrites (Fig. $6 E$ ) (from $0.045 \pm 0.036$ during 4-d NV to $0.078 \pm 0.035$, $p=0.011$ ), and differed significantly from that of controls during 4-d MD (Fig. 6E) $(0.049 \pm$ $0.036, p=0.042)$. Similarly, MD-induced change in spine elimination $\left(\Delta_{E}\right)$ in sh95expressing dendrites was significantly larger than control dendrites after 4-d MD (Fig. 6F) (mean \pm SEM; sh95 $0.033 \pm 0.011$ vs. ctrl $-0.006 \pm 0.015 ; 2$-way ANOVA: $F_{\mathrm{g}}=2.630, F_{\mathrm{t}}=$ 2.708, $F_{g^{*}}=5.854, p=0.027$ ), but not after $1-d$ MD (ctrl $-0.001 \pm 0.009$ vs. sh95 $-0.005 \pm$ $0.0085, p>0.9)$.

Spine formation ratios were comparable during 1-d and 4-d NV between the groups (Fig. $6 G$ ) (mean \pm SD; GLME: $F_{\mathrm{g}}=0.004, F_{\mathrm{md}}=10.503, F_{\mathrm{t}}=1.660, F_{\mathrm{g}^{*} \mathrm{md}}=0.475$; ctrl: NV 0.057 \pm 0.038 vs. MD 0.039 $\pm 0.033, p=0.064$; sh95: NV $0.043 \pm 0.028$ vs. MD $0.034 \pm 0.025, p$ $=0.260$ ), and remained stable after 1-d (Fig. 6G) or 4-d MD (Fig. 6H) in both sh95 (4-d NV $0.064 \pm 0.040$ vs MD $0.047 \pm 0.035, p=0.154$ ) and control groups (4-d NV $0.056 \pm 0.039$ vs. MD $0.035 \pm 0.038, p=0.068)$. There were also no significant differences in $\Delta_{\mathrm{F}}$ values between groups for 1 - $d$ or 4 -d periods (Fig. $5 /$ ) (ctrl $-0.017 \pm 0.001$ vs sh95 $-0.021 \pm 0.012$, $p>0.9$ ). Although spine formation rate remained stable in sh95 dendrites during MD, spine elimination was increased, similar to KO dendrites. Thus, we conclude that the effect of PSD-95 in regulating experience-dependent spine stability is cell-autonomous. 


\subsection{Discussion}

Postsynaptic spines and presynaptic boutons are the loci for structural reorganizations accompanying the plastic changes in the cortex. In the present study, we used awake invivo two photon microscopy to examine the role of an important post-synaptic molecular player - PSD-95, in dendritic spine stability in the context of ODP. In V1, PSD-95 expression levels begin to increase at eye opening and reach a plateau at $\sim$ P35 (Huang et al., 2015). Increase in PSD-95 levels progressively drives AMPAR-silent synapsematuration and terminates CP for ODP, while absence of PSD-95 results in juvenile like ODP throughout adulthood (Favaro et al., 2018; Huang et al., 2015). Here we examined experience-dependent spine dynamics in adult PSD-95-deficient mice to find out the structural correlates of juvenile-like ODP in these mice. We have done all the experiments in awake animals to exclude potential anesthesia effects on spine dynamics (Briner et al., 2010; Kaech et al., 1999; Yang et al., 2011).

We found that 4-d MD leads to an almost two-fold increase in spine elimination at the apical dendrites of L2/3 PNs in adult (P64-P79) PSD-95 KO mice, which is comparable to what has been reported in L2/3 apical dendrites of juvenile (P28) WT mice after a 3-d MD (Sun et al., 2019). Moreover, a similar increase in spine elimination rate has been observed in L5 apical dendrites in juvenile WT mice after a 3-d MD (Zhou et al., 2017). In light of these recent anatomical studies, it is now compelling to suggest that reduction of deprived eye responses in ODP experiments is mediated by increased spine pruning at PNs located above and below L4 in binocular V1. Furthermore, we show that in adult PSD-95 WT mice, spine dynamics at $L 2 / 3$ apical dendrites remain stable during $M D$, consistent with a previous study (Hofer et al., 2009). In contrast to juvenile mice, a prolonged MD (7-d) is needed to induce OD-shifts in older mice (Lehmann and Löwel, 2008), and the shifts are mediated by enhancement of open-eye responses (Frenkel et al., 2006; Sato and Stryker, 2008), which seem to correlate with increased spine formation in L5 (Hofer et al., 2009). Taken together, our earlier results from optical imaging of intrinsic signals (Huang et al., 2015) and current findings from repeated two-photon imaging all point clearly to the notion that experiencedependent plasticity of adult PSD-95-deficient mice resemble the ODP seen in juvenile mice, both physiologically and anatomically (Gordon and Stryker, 1996; Sun et al., 2019).

In V1 of PSD-95 KO mice, $\sim 50 \%$ of synapses do not mature and remain AMPAR-silent into adulthood (Huang et al., 2015). Since spines shapes in PSD-95 KO mice are indistinguishable from WT controls (Béïque et al., 2006; Migaud et al., 1998), AMPAR-silent synapses are most likely morphologically indistinguishable if investigated with static 
microscopy tools. In a longitudinal spine imaging study using hippocampal slice cultures, acute knockdown of PSD-95 was shown to increase spine turnover and impair spine stabilization after LTP induction (Ehrlich et al., 2007). Also, new spines which do not acquire PSD-95 puncta are more likely to be eliminated (Cane et al., 2014). Consistent with these observations, we now show that in mature brain PSD-95 is required to maintain the stability of the newly formed connections when the circuit is challenged by sensory deprivation. Moreover, silencing PSD-95 expression in V1 at birth or in adulthood increases AMPARsilent synapse fractions cell-autonomously, and leads to enhancement of ODP similar to age-matched KO mice (Huang et al., 2015). Thus, we have also tested the experiencedependent spine dynamics in PSD-95 lacking neurons in the wildtype environment. Similar to KO mice, here too we have seen an increase in spine elimination in sh95-expressing neurons after 4-d MD. We therefore conclude that experience-dependent spine dynamics are regulated by PSD-95 cell-autonomously.

In addition to being enhanced, OD-shifts are also more transient in adult PSD-95 KO mice and return to normal only after $2 \mathrm{~d}$ of reopening the deprived eye (Huang et al., 2015), similar to juvenile mice (Hofer et al., 2006; Kaneko et al., 2008). We tested whether prompt recovery of functional changes in KOs can also be explained at the structural level. Interestingly, $2 \mathrm{~d}$ after reopening the deprived eye, spine dynamics in PSD-95 KOs were not significantly different from WT controls, suggesting that prompt recovery from MD in KO mice is not accomplished through circuit rewiring. Studies investigating spine dynamics during recovery from $M D$ in juvenile mice, have also reported mixed results so far: in layer 2/3, 2 d recovery reverts MD-induced changes in dynamics back to baseline (Sun et al., 2019); however, in layer 5, MD-induced increase in elimination rate persists even $4 \mathrm{~d}$ after restoration the binocular vision (Zhou et al., 2017). Here, after $4 \mathrm{~d}$ of reopening we did not see any significant differences in spine dynamics between genotypes. However, intragroup comparisons of spine dynamics to baseline (pre-MD) phases revealed that in KOs, spine elimination ratios were still significantly higher than baseline. It is likely that after the reopening spines receiving the spared-eye input get eliminated, which leads to the recovery of the contralateral dominance, although future experiments would need to confirm this. Spine formation ratios during the recovery were, on the other hand, comparable to the baseline condition. Thus, spine dynamics fail to explain the faster recovery of OD in PSD$95 \mathrm{KO}$ mice upon reopening, suggesting that synaptic plasticity mechanisms such as LTP/LTD, must be at play in parallel. It is, in fact, known that LTP is enhanced in PSD-95 KO neurons (Béïque et al., 2006; Carlisle et al., 2008; Migaud et al., 1998), which makes it tempting to argue that in KOs during 2-d recovery, spines that receive input preferably from 
previously deprived eye undergo prompt LTP, bringing deprived eye responses in V1 back to normal without a need for new spine formation.

In conclusion, here we demonstrate that 4-d MD leads to a drastic MD-dependent spine elimination in PSD-95-deficient L2/3 PNs in adult mice brain, which provides an anatomical correlate for the enhanced juvenile-like ODP mediated by reduction of deprived eye responses. Such structural dynamics are reminiscent of highly plastic juvenile mice brain (Sun et al., 2019; Zhou et al., 2017), which is also characterized by lower PSD-95 expression and high AMPAR-silent synapse ratios (Favaro et al., 2018; Funahashi et al., 2013; Han et al., 2017; Huang et al., 2015). Interestingly, newly formed spines in PSD-95 $\mathrm{KO}$ mice are particularly vulnerable to MD-dependent circuit reorganization, which points towards the interplay of molecular machinery and experience in shaping the neural circuitry in the cortex. Collectively these results place PSD-95 as an important link between structure and the function of the post-synapse, and further consolidate the concept of silent synapsedependent neural network stabilization during CPs. 


\section{Acknowledgements}

We thank Prof. Dr. C. Schwarz and Dr. B. Joachimsthaler for sharing their awake twophoton imaging routine and spine analysis scripts; J.D. Flórez Weidinger and A. Schmidt for the motion correction script; Dr. N. M. Moreno for showing us in utero electroporation surgery; and M. Schink for excellent animal care. This work was supported by the German Research Foundation through the Collaborative Research Center 889 "Cellular Mechanisms of Sensory Processing" [to S.L. (Project B5), O.M.S. (Project B3), and J.S. (Project C7) ] and by the Federal Ministry of Education and Research, Germany (Grant 01GQ0810 to S.L.).

\section{Author Contributions}

Study design by R.Y., A.T., S.L., and O.M.S. Data collection by R.Y. Data analysis by R.Y. Data interpretation by R.Y., S.L., and O.M.S. Manuscript preparation by R.Y.

\section{Competing interests}

The authors declare no competing interests.

\section{Correspondence}

sloewel@gwdg.de 


\section{Loss of neurogranin in the visual cortex preserves juvenile-like ocular dominance plasticity into adulthood.}

Rashad Yusifov ${ }^{1,2}$, Thomas Kräll ${ }^{1}$, Oliver M. Schlüter ${ }^{2,3,4}$, Siegrid Löwel ${ }^{1,2,5}$

${ }^{1}$ Department of Systems Neuroscience, Johann-Friedrich-Blumenbach Institut für Zoologie und Anthropologie, Universität Göttingen, Göttingen, Germany

${ }^{2}$ Collaborative Research Center 889, Universität Göttingen, Göttingen, Germany

${ }^{3}$ Department of Neuroscience, University of Pittsburgh, Pittsburgh, PA, USA

${ }^{4}$ Department of Psychiatry and Psychotherapy, University Medical Center Göttingen, Göttingen, Germany

${ }^{5}$ Campus Institute for Dynamics of Biological Networks, Universität Göttingen, Göttingen, Germany

Author contribution of Rashad Yusifov:

- Design (together with Siegrid Löwel, Oliver M. Schlüter),

- Performance of the experiments (together with Thomas Kräll for Fig. 2 and Fig. 4)

- Analysis of all the data

- Preparation of the manuscript

This manuscript has been prepared for publication. 


\subsection{Abstract}

Critical periods are characterized as developmentally restricted time windows of heightened cortical plasticity and experience-dependent optimization of neural circuits. Factors governing the duration of critical periods are still not fully resolved. Ocular dominance plasticity (ODP) in the primary visual cortex (V1) has been the mainstay model for investigating the molecular and cellular underpinnings of critical period plasticity. Maturation of glutamatergic connections via un-silencing of AMPAR-silent synapses in the visual cortex has been shown to play a key role in bringing the critical period for ODP to a halt. More recently it was demonstrated that neurogranin, a postsynaptic calmodulin-binding protein enriched in the cortex and hippocampus, is required for the experience-dependent maturation of AMPAR-silent synapses in juvenile mice (Han et al., 2017). Here we show that postnatal knock-down of neurogranin expression in V1 prevents the closure of critical period and preserves the juvenile-like ODP into adulthood, while also impairing the visual acuity. In adult neurogranin ( P65) knock-down mice a brief MD (4-d) led to a reduction in contralateral deprived eye responses shifting the ocular dominance towards the open eye, an ODP phenotype typical for juvenile mice. Next, we have tested the ODP in an older ( P130) group of mice, at an age when even 7-d MD is not anymore sufficient to induce plastic changes in the wild-type standard cage-raised mice. We found that after 7-d MD neurogranin knock-down mice show significantly reduced ocular dominance values compared to controls, suggesting that in the absence of neurogranin, ODP remains enhanced well beyond the critical period. Finally, we tested the basic visual capabilities of neurogranin knockdown-mice in the visual water task (VWT) and virtual reality optomotor setup. The maximum visual acuity of neurogranin knockdown mice was impaired at the VWT, while the optomotor reflexes were similar to control mice. 


\subsection{Introduction}

Brain plasticity is heightened during early development and allows experience-dependent refinement of synaptic transmission. These limited time windows, defined as critical periods (CP), are observed in several functional domains in the brain (Brainard and Doupe, 2002; Feldman et al., 1996; Roberts et al., 2010; Wiesel and Hubel, 1963b) . Developmental decrease of brain plasticity has been best described by the experience dependent decline of ocular dominance plasticity (ODP) in the primary visual cortex (V1) (Espinosa and Stryker, 2012; Levelt and Hübener, 2012). In mice, binocular part of V1 receives afferents from both eyes, but is dominated by the contralateral eye inputs (Dräger, 1975). During the critical period for ODP, a brief monocular deprivation (MD) induces rapid plastic changes leading to a reduction of responsiveness to the deprived eye in the contralateral V1 (Dräger, 1978; Gordon and Stryker, 1996). Beyond the age of critical period, in adult standard cageraised mice, plastic changes are inducible only after a prolonged MD (Lehmann and Löwel, 2008), which are also mechanistically different from those of juvenile mice (Sato and Stryker, 2008; Sawtell et al., 2003).

Normal experience is crucial during the CPs for optimal neural wiring and functional maturation of the cortex (Wang et al., 2010), while such experience-dependent maturation of the glutamatergic synapses also promotes the decline in neural plasticity (Favaro et al., 2018; Huang et al., 2015). In the beginning of the CP for ODP, $\sim 50 \%$ of the post-synapses in layer 4 to layer 2/3 circuitry of V1 lack AMPA receptors (AMPAR) (Favaro et al., 2018; Huang et al., 2015), defined as AMPAR-silent synapses (Isaac et al., 1995; Liao et al., 1995). After the eye opening in mice, normal sensory experience initiates the strengthening of the favored connections through insertion of AMPARs in the post-synaptic membrane of AMPAR-silent synapses (Favaro et al., 2018; Funahashi et al., 2013; Han et al., 2017). Such experience dependent reduction in the fraction of AMPAR-silent synapses has been linked to the closure of critical period for ODP (Favaro et al., 2018; Huang et al., 2015), Perturbing the molecular machinery of AMPAR trafficking by deleting postsynaptic density (PSD) proteins, PSD-93 and PSD-95 has a drastic effect on the pace of AMPAR-silent synapse maturation and CP closure for ODP (Favaro et al., 2018; Huang et al., 2015). Deletion of PSD-95 prevents the experience-dependent maturation of AMPAR-silent synapses, and results in lifelong preservation of CP-like ODP, while in PSD-93-deficient mice the $\mathrm{CP}$ is precociously terminated due to faster maturation of AMPAR-silent synapses (Favaro et al., 2018; Huang et al., 2015).

The fates of synapses during development is dependent on experience-dependent stimulation and dictated by whether they will be incorporated into a functional circuit (Katz 
and Shatz, 1996; Löwel and Singer, 1992). In the post-synapse, activation of $\mathrm{Ca}^{2+} /$ calmodulin (CaM)-dependent protein kinase II (CaMKII) or calcineurin defines whether the synapse will undergo long-term potentiation (LTP) (Malinow et al., 1988; Meyer et al., 1992; Silva et al., 1992) or long-term depression (LTD) (Mulkey et al., 1994; Torii et al., 1995), respectively. Neurogranin $(\mathrm{Ng})$ is an important post-synaptic molecule enriched in the cortex and hippocampus (Represa et al., 1990; Singec et al., 2004; Watson et al., 1992) that regulates the spatiotemporal abundance of CaM in the molecular cascade leading to LTP/LTD (Gaertner et al., 2004; Petersen and Gerges, 2015). Presence of neurogranin ensures the availability of CaM at the postsynaptic side of synapse (Petersen and Gerges, 2015). Upon $\mathrm{Ca}^{2+}$ influx, $\mathrm{Ca}^{2+} / \mathrm{CaM}$ complex activates CaMKII dependent processes leading to LTP via the incorporation AMPARs at the post-synapse (Malinow et al., 1988). It has been recently demonstrated that neurogranin is involved in the experience dependent maturation of AMPAR-silent synapses in the visual cortex (Han et al., 2017). Its deletion leads to elevated fraction of AMPAR-silent synapses reminiscent of PSD-95-deficient mice (Huang et al., 2015), and increases the spine loss during the maturation (Han et al., 2017). Moreover, its deletion also shifts the synaptic plasticity towards enhanced LTD and diminished LTP (Han et al., 2017; Hwang et al., 2018). These findings led us hypothesize that neurogranin is likely involved in limiting the CP plasticity for ODP, and that reducing its postnatal expression will extend the CP into adulthood.

To answer this question we examined the role of neurogranin in cortical plasticity by silencing its expression at birth via delivery of small-hairpin RNA (shNg) into V1. Using intrinsic signal imaging (Cang et al., 2005a; Kalatsky and Stryker, 2003), we have assessed the ODP of shNg mice after 4- or 7-d MD periods in two-different age groups during adulthood. We have also investigated the impact of neurogranin on the visual capabilities of adult mice. We found that in the absence of neurogranin, juvenile like ODP was preserved well beyond the normal age of critical period closure. In addition, we show that visual acuity is impaired in shNg mice in the visual water task, but the maximum threshold reflex and contrast sensitivities in the virtual reality optomotor task were not affected. 


\subsection{Methods}

\section{Experimental Animals}

C57BL/6J mice were obtained from the mouse colony of the central animal facility, University Medical Center Göttingen, and housed with a $12 \mathrm{~h}$ light/dark cycle in standard cages $(26 \times 20 \times 14 \mathrm{~cm})$. Food and water were available ad libitum. All experimental procedures were approved by the local government (Niedersächsisches Landesamt für Verbraucherschutz und Lebensmittelsicherheit). Both sexes of animals were included in the study. The indicated age of mice is at the day of the optical imaging experiment.

\section{P0 virus injections}

Adeno-associated virus (AAV) expressing small-hairpin RNA for silencing neurogranin expression (shNg), and a control AAV expressing shRNA that targeted the luciferase gene (shLc), both with CAG-driven GFP sequence (Han et al., 2017), were delivered to P0-P2 mice as described before (Huang et al., 2015). Pups were anesthetized on ice for 5 min and immobilized with a holder on a custom-made injection table, where the surface temperature was kept at $4{ }^{\circ} \mathrm{C}$. The sagittal and lambdoid suture (lambda) intersection was visually identified through the skin. AAVs were injected at two positions ( $100 \mathrm{~nL}$ each) bilaterally into V1 (from lambda, in mm: AP +0.1, ML \pm 1.25 , DV -0.8 and AP +0.3, ML \pm 1.8 , DV -0.8) using a glass capillary with a Nanoject II microinjector (Drummond). Mice recovered from anesthesia on a heating pad kept at $37^{\circ} \mathrm{C}$, and were afterwards returned to their home cages.

\section{Monocular Deprivation}

The right eye was deprived for $4 \mathrm{~d}$ in young adult mice (P45-P79), and for $7 \mathrm{~d}$ in the older mice (P121-145) according to published protocols (Gordon and Stryker, 1996). Briefly, mice were anesthetized with $2 \%(\mathrm{vol} / \mathrm{vol})$ isoflurane in a mixture of $\mathrm{O}_{2}: \mathrm{N}_{2} \mathrm{O}(1: 1)$ and received carprofen $(5 \mathrm{mg} / \mathrm{kg})$ injections for analgesia. Eyelid margins were trimmed and sutured together with two mattress sutures. Animals were inspected daily to ensure that the eye remained closed, otherwise were excluded from further experiments. Deprived eye was opened immediately before the optical imaging experiment. 


\section{Virtual reality optomotor test}

Both the spatial frequency and the contrast sensitivity threshold of the optomotor reflex of mice were assessed by the virtual reality optomotor system (Prusky et al., 2004). Freely moving animals were positioned on a small elevated platform surrounded by four computer monitors $(33.5 \times 26.5 \mathrm{~cm})$ showing moving sine wave gratings of various spatial frequencies and contrasts, which formed a virtual rotating cylinder. Mice reflexively track the rotating gratings by small head movements as long as they can see the gratings. Spatial frequency at maximum contrast, as well as contrast at six different spatial frequencies [0.031, 0.064, $0.092,0.103,0.192$, and 0.272 cycles/degree $(c / d)]$ were varied by the experimenter until the threshold of tracking was determined. Contrast sensitivity thresholds of the optomotor reflex measured in percent were converted into Michelson contrasts. Mice were measured once before MD and daily during the duration of MD (or no-MD) for $4 \mathrm{~d}$.

\section{Visual Water Task (VWT)}

The VWT is a visual discrimination task for assessing the visual acuity in rodents (Prusky et al., 2000). The apparatus consists of a trapezoidal-shaped water-filled pool with two adjacent monitors, a divider defining the choice line between two monitors, and a transparent escape platform hidden below the rewarded monitor (Cerebral Mechanics). During the training phase, the monitor displaying vertical black and white grating at $100 \%$ contrast was associated with an escape platform to reinforce swimming towards the grating. Mice were initially trained to distinguish a low spatial frequency vertical sine wave grating (0.086 cycles/deg) from isoluminant gray. The position of the rewarded stimulus was alternated between left and right monitors in a pseudo-random sequence over the training and testing trials. Once mice reached $90 \%$ accuracy in three consecutive blocks (each 10 trials) during the training phase, maximum discrimination threshold was tested by increasing the spatial frequency of the grating (in steps 0.017 cycles/deg) until performance fell below $70 \%$ accuracy. Mice were tested for similar number of trials (> 956 trials) to account for improvements in visual acuity due to long-term training (Hosang et al., 2018). Three consecutive failures to reach $70 \%$ accuracy at similar frequencies ( \pm 1 step) were taken as the visual acuity limit. Spatial frequencies one step below the limit were averaged and taken as the "visual acuity" of mouse. 


\section{Optical Imaging of Intrinsic Signals and Visual Stimuli.}

Surgery. Surgical preparations for optical imaging were performed as published before (Greifzu et al., 2014). In short, following the box anesthesia with $2 \%$ halothane in a mixture of $1: 1 \mathrm{O}_{2} / \mathrm{N}_{2} \mathrm{O}$, the mice were injected with carprofen (5 mg/kg, s.c.; Zoetis), atropine (5 mg/kg, s.c.; Franz Köhler), dexamethasone (8 mg/kg, s.c.; Merck), and chlorprothixene (5 $\mathrm{mg} / \mathrm{kg}$, i.m.; Sigma-Aldrich). The mice were placed in a stereotaxic frame and the body temperature was maintained at $37{ }^{\circ} \mathrm{C}$. The heart rate was monitored throughout the experiment. Anesthesia was kept at $0.6-0.8 \%$ halothane in a mixture of $1: 1 \mathrm{O}_{2} / \mathrm{N}_{2} \mathrm{O}$ applied through a tube over the nose. Lidocaine ( $2 \%$ xylocaine gel, Astra Zeneca ) was applied locally before the skin incision that exposed the left hemisphere. Exposed skull was covered with low-melting point agarose $(2.5 \%$ in $0.9 \% \mathrm{NaCl})$ and a glass coverslip was placed over the visual cortex.

Data acquisition and visual stimulation. Mouse cortical responses were recorded through the skull using the "Fourier"-imaging method (Kalatsky and Stryker, 2003) optimized for the assessment of ODP (Cang et al., 2005a). Images of V1 intrinsic signals were captured on a CCD-camera (Dalsa 1M30) using a 135x50 mm tandem lens configuration (Nikon), under red illumination light $(610 \pm 10 \mathrm{~nm})$. Frames were acquired at a rate of $30 \mathrm{~Hz}$, temporally binned to $7.5 \mathrm{~Hz}$, and stored as $512 \times 512$ pixel images after spatial binning of the camera image. White drifting horizontal bars $\left(20^{\circ}\right.$ wide) were presented on a high refresh rate monitor (BenQ, BL-2400B, 19 inch) positioned $25 \mathrm{~cm}$ from the eyes. For imaging ODP, the visual stimulus was restricted to the binocular visual field of the left primary visual cortex $\left(\mathrm{V} 1 ;-5^{\circ}\right.$ to $+15^{\circ}$ azimuth), and mice were stimulated through either the left or the right eye in alternation to assess the OD. For visualizing elevation and azimuth maps, we stimulated the contralateral eye using full-field stimuli extending $94^{\circ}$ horizontally and $62^{\circ}$ vertically (Cang et al., 2005b).

Data Analysis. The amplitude component of the optical signal represents the intensity of neuronal activation (expressed as fractional change in reflectance $10^{-4}$ ) and was used to calculate the $\mathrm{OD}$. Maps obtained from at least three blocks of imaging per animal were averaged to compute the $O D$ index $(O D I)$ as $(C-I) /(C+I)$, with $C$ and I representing the response magnitudes of each pixel to visual stimulation of the contralateral and ipsilateral eye, respectively. The ODI ranges from -1 to 1 , with negative values representing ipsilateral and positive values representing contralateral dominance. V1 activity maps depicted in the Results section are the averages of at least three maps from individual animals, except for azimuth and elevation maps which were obtained from one block of stimulation. 


\section{Perfusion and brain sectioning.}

Immediately after optical imaging experiments, all mice were deeply anesthetized with an intraperitoneal injection of chloral hydrate $(3 \mathrm{~g} / \mathrm{kg})$ and buprenorphine $(1 \mathrm{mg} / \mathrm{kg})$ mixture and were perfused transcardially with $0.9 \%$ saline solution ( $\mathrm{pH} 7.4,0.1 \mathrm{M}$ heparin) for $2 \mathrm{~min}$ followed by 5 minutes of $4 \%$ paraformaldehyde $(\mathrm{pH} 7.4)$ in $0.1 \mathrm{M}$ phosphate buffered saline (PBS). The brain was removed and post-fixed in $4 \%$ paraformaldehyde $(\mathrm{pH} 7.4)$ for $1 \mathrm{~d}$, and then transferred to a $10 \%$ sucrose solution in PBS for $1 \mathrm{~d}$ followed by a $30 \%$ sucrose solution in PBS for 1 or $2 \mathrm{~d}$. The brains were then frozen in methylbutane and stored at -80 ${ }^{\circ} \mathrm{C}$. Coronal brain sections were cut on a microtome at $50 \mu \mathrm{m}$, and the slices from $\mathrm{V} 1$ were inspected under a fluorescence microscope (Axioskop 50, Carl Zeiss). Animals with no GFP expression in the binocular V1 were excluded from further analyses (see Supplementary Figure 1).

\section{Statistical analysis.}

All intragroup and intergroup comparisons were analyzed using ANOVA with Bonferroni's corrections or two-tailed t-tests, as indicated in the text. The normality of the data was tested using Shapiro-Wilk test, and the data are represented as means \pm SEM The levels of significance were set as ${ }^{*} p<0.05,{ }^{* *} p<0.01$, and ${ }^{* *} p<0.001$. 


\subsection{Results}

\section{The juvenile form of ocular dominance plasticity is preserved in neurogranin knockdown mice during adulthood.}

4-d MD results in rapid reduction of deprived eye responses in the binocular V1 in juvenile standard cage-raised WT mice up to P32, but not beyond this age (Gordon and Stryker, 1996). As neurogranin was shown to mediate the maturation of AMPAR-silent synapses in juvenile mice (Han et al., 2017), we investigated whether postnatal silencing (at P0-P2) of neurogranin expression in V1 would preserve the juvenile-like ODP into adulthood. To this end we have optically assessed the ODP in control (shLc) and neurogranin knock-down (shNg) mice after 4-d MD or no MD during adulthood. In adult shNg mice without MD (P50P80: P63), V1 was dominated by visual input from the contralateral eye similar to control mice (P49-P80; P63) (Fig. 1 A). V1 remained dominated by the contralateral (deprived eye) input in the control mice with 4-d MD (P57-P83: P65), whereas in shNg mice (P57P83: P67) there was a strong reduction in deprived eye responses (Fig. 1A). In shNg mice without MD, the two-dimensional OD map showed warm colors, indicating contralateral dominance, comparable to control mice. In contrast, after 4-d MD colder colors prevailed the OD map and the OD histogram was shifted to the left.

Quantitative analysis of OD indices (Fig. 1B) in the control group revealed a moderate but non-significant difference between no MD $(0.244 \pm 0.022)$ and MD mice $(0.164 \pm 0.011 ; p$ $=0.057$, ANOVA), suggesting lack of ODP after 4-d MD in adult control mice. In contrast, $\mathrm{OD}$ indices of shNg mice with 4-d MD $(0.042 \pm 0.022)$ were strongly reduced in comparison to shNg mice with no MD $(0.196 \pm 0.023 ; p<0.001$, ANOVA $)$, and in comparison to control groups with $(P<0.001)$ and without $4-d M D(p<0.001)$ (Fig. 1B). Furthermore, in shNg mice without MD, V1 activation after contralateral-eye stimulation was stronger than that of ipsieye stimulation (Fig. 1C) (1.037 \pm 0.082 vs. $0.729 \pm 0.056 ; p=0.002$, ANOVA), similar to control mice with no MD (contra/ipsi: $1.424 \pm 0.176$ versus $0.889 \pm 0.092, p<0.001$ ) or 4 $\mathrm{d}$ MD (contra/ipsi: $1.364 \pm 0.133$ versus $1.008 \pm 0.092, p<0.001$ ). This was no longer the case in shNg mice with 4-d MD, where deprived eye responses were low $(0.855 \pm 0.074)$ and comparable to ipsi-eye responses $(0.790 \pm 0.070, p>0.9)$ (Fig. 1C). These results show that in adult shNg mice, OD shifts can be induced by a brief MD (4-d), which is one of the hallmarks of CP plasticity (Espinosa and Stryker, 2012). Additionally, after 4-d MD, deprived eye responses are reduced to similar levels as ipsi-eye responses, major defining feature of ODP during CP. 


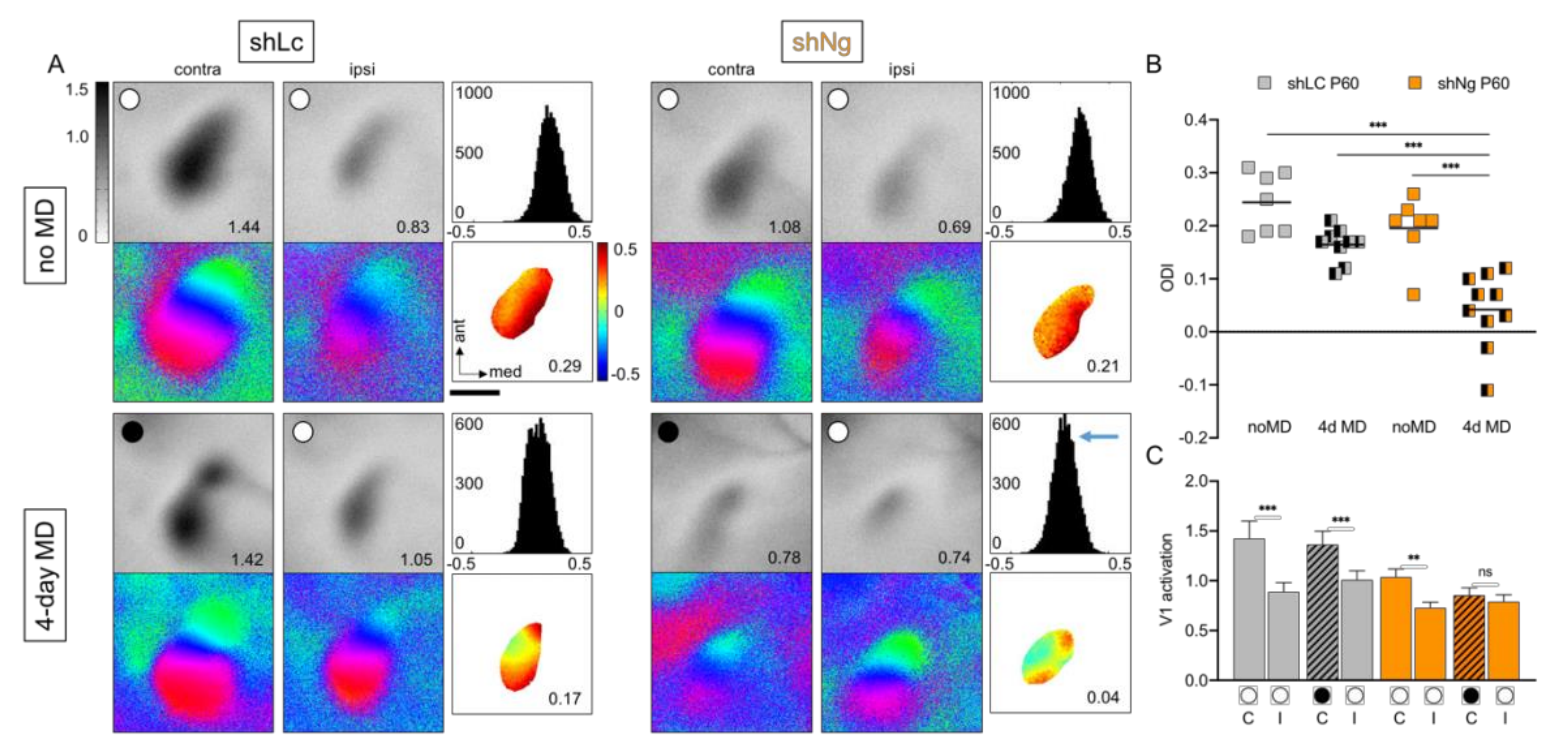

Figure 1. Silencing neurogranin expression postnatally prevents the closure of the critical period for ocular dominance plasticity. (A) Optically recorded activity maps of the contralateral (contra) and ipsilateral (ipsi) eye in binocular $\mathrm{V} 1$ of $\sim \mathrm{P} 60 \mathrm{shLc}$ and $\mathrm{shNg}$ transduced mice with and without 4-d MD. Grayscale-coded response magnitude maps [numbers in the bottom right corner correspond to the quantified V1 activation for the depicted map (expressed as fractional change in reflectance $\times 10^{-4}$ ); see gray scale, top left]; o, open eyes; •, deprived eyes; color-coded maps of retinotopy (bottom rows), histogram of OD scores (top right of panels, left axis: number of included pixels), and colorcoded OD map (bottom right including average ODI value) are illustrated (scale bar, $1 \mathrm{~mm}$ ). In shLc and shNg mice without MD, activities evoked by stimulation of the contralateral eye were stronger (darker patches) than those of the ipsilateral eye, the average ODI was positive, and warm colors prevailed in the OD maps, indicating contralateral dominance. After 4-d MD, in shNg mice activity evoked by stimulation of the contralateral eye became equally strong as that of the ipsilateral eye, the average ODI was closer to 0 , and the cold colors prevailed in the OD maps, indicating an OD shift. In contrast, OD shifts were absent in shLc (control) mice after 4-d MD. (B), (C) Quantification of V1 activity maps. (B) Optically imaged ODls of shLc (gray) and shNg mice (orange) after no MD (full square) or 4-d MD (half filled). Symbols represent ODI values of individual animals. Means are marked by horizontal lines. (C) Mean of max. V1 activation elicited by stimulation of the contralateral (C) or ipsilateral (I) eye with and without MD (black filled circle indicates MD eye). * $p<0.05$, ${ }^{* *} p<0.01$, and ${ }^{* * *} p<0.001$. 
Ocular dominance plasticity is enhanced in older neurogranin knockdown mice >P110.

Beyond $\mathrm{CP}$, prolonging the MD duration from 4 to 7 days can still induce $\mathrm{OD}$ shifts in standard cage-raised adult WT mice until they reach the age of P110 (Lehmann and Löwel, 2008). In contrast to juvenile-like ODP, these shifts are mediated by increases in open eye responses, and the deprived-eye responses remain stable (Lehmann and Löwel, 2008; Sato and Stryker, 2008; Sawtell et al., 2003). Next, we have tested whether reducing neurogranin levels postnatally can preserve ODP even beyond $\mathrm{P} 110$. To this end, we have assessed the ODP in an older group of control (P125-P142: P135) and shNg mice (P125P149: P132). After 7-d MD V1 was still dominated by the contralateral eye in control mice, and the average ODI of group was $0.213 \pm 0.025$. In shNg group with 7-d MD, however, ODI values were more variable, but were nevertheless significantly reduced $(0.0875 \pm$ 0.0378 ) compared to control group (Welch's t-test, $p=0.020$ ).

A

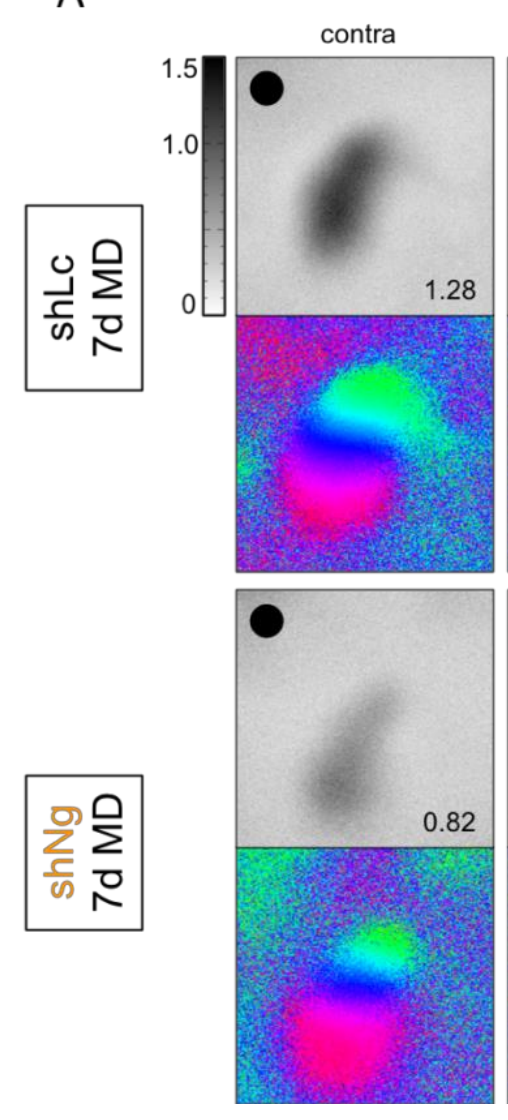

ipsi
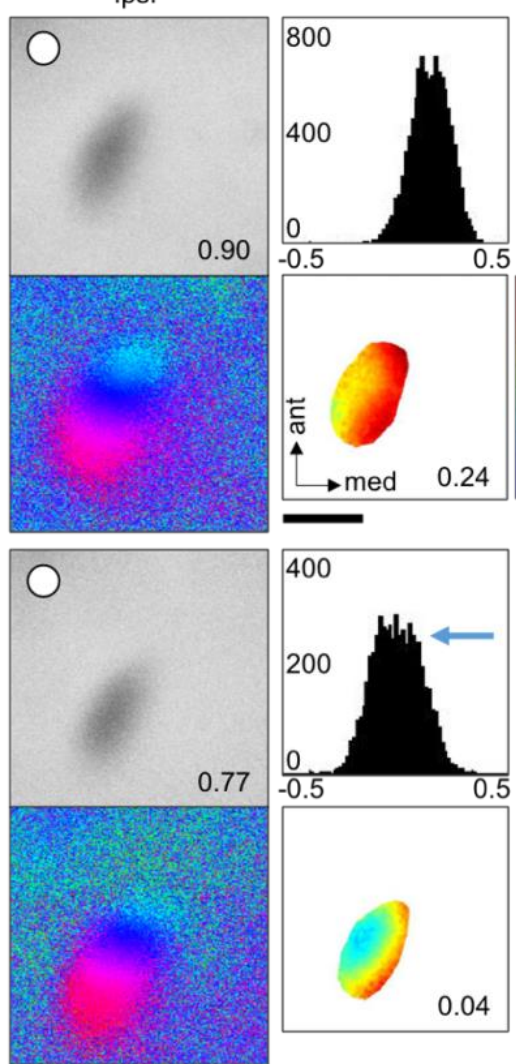

B

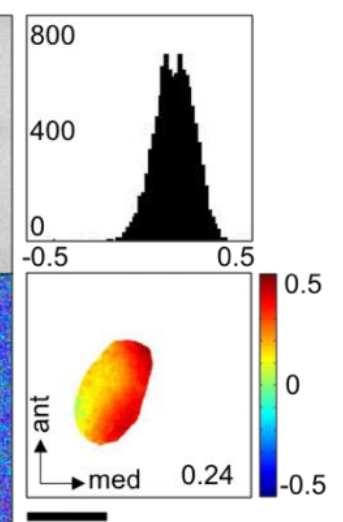

C
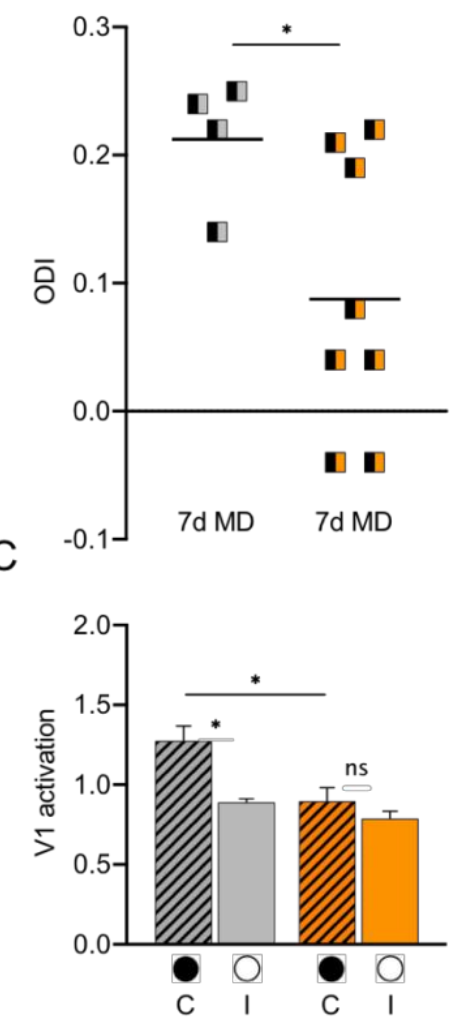

Figure 2. ODP is preserved well beyond the CP (>P110) in shNg mice. (A), Optically recorded activity maps of the contralateral (contra) and ipsilateral (ipsi) eye in binocular V1 of shLc and shNg transduced mice following 7-d MD. Layout as in Figure 1. Grayscalecoded response magnitude maps; ○, open eyes; •, deprived eyes; color-coded maps of retinotopy (bottom rows), histogram of OD scores, and color-coded OD map are illustrated 
(scale bar, $1 \mathrm{~mm}$ ). In shLc mice (P135) with 7-d MD, responses evoked by stimulation of the contralateral eye were stronger (darker patches) than those of the ipsilateral eye, the average ODI was positive, and warm colors prevailed in the OD maps, indicating contralateral dominance. In shNg mice ( P132) activity evoked by stimulation of the contralateral eye was equally strong as that of the ipsilateral eye, the average ODI was closer to 0, and the colder colors prevailed in the OD maps. (B), (C), Quantification of V1 activity maps. (B) Optically imaged ODIs of shLC (gray) and shNg mice (orange) after or 7d MD. Symbols represent ODI values of individual animals. Means are marked by horizontal lines. (C) Mean of max. V1 activation elicited by stimulation of the contralateral (C) or ipsilateral (I) eye (black filled circle indicates MD eye). ${ }^{*} p<0.05$

Quantification of V1 responses elicited by deprived-and open-eye stimulation revealed that, after 7-d MD V1 was dominated by deprived-eye responses in control mice (contra/ipsi: $1.275 \pm 0.092$ vs. $0.890 \pm 0.023 ; p=0.037$, two-way ANOVA with Bonferroni corrections), as expected (Lehmann and Löwel, 2008). In shNg mice, however, deprived-eye responses $(0.896 \pm 0.086)$ were comparable to the open-eye responses $(0.789 \pm 0.045 ; p>0.9)$, and significantly lower than contra-eye responses of control mice $(p=0.014)$. These results suggest that ODP is enhanced in shNg even beyond P110, and the OD shifts are due to the reduced deprived-eye responses similar to juvenile-like ODP.

\section{The Basic Organization of Visual Cortex Is Normal In ShNg Mice.}

To test whether basic organization of $\mathrm{V} 1$ in shNg and shLc transduced mice are normal, we analyzed V1 retinotopic maps, activated by either vertically (elevation) or horizontally (azimuth) drifting bars presented to the contralateral eye (Fig. 3A) (Cang et al., 2005b). The magnitude of the V1 responses after elevation (Fig. 3B) stimulation were significantly ( $p=$ 0.006 , Two-tailed t-test) lower in shNg group $(n=7 ; 1.87 \pm 0.285)$ compared to controls $(n$ $=7 ; 2.27 \pm 0.145)$, while for azimuth maps did not differ significantly $(p=0.604)$ between groups (Fig. 3D) (shLc/shNg: $1.96 \pm 0.447$ vs. $1.82 \pm 0.370)$, consistent with previous reports of lowered field excitatory postsynaptic potential in neurogranin knockout mice (Han et al., 2017; Pak et al., 2000).

Map quality/scatter (Fig. 3C and 3E) was analyzed by calculating the standard deviation for visual field position differences of each pixel between its surrounding 25 pixels (Cang et al., 2005b). The quality of retinotopic maps did not differ significantly between groups for elevation (shLc/shNg: $1.92 \pm 0.86$ vs. $3.74 \pm 0.85 ; p=0.067$ ) or azimuth maps (shLc/shNg: 
$12.2 \pm 1.45$ vs. $12.3 \pm 1.07 ; p>0.9$ ), suggesting that the basic functional organization of $\mathrm{V} 1$ is not disturbed by silencing neurogranin expression .

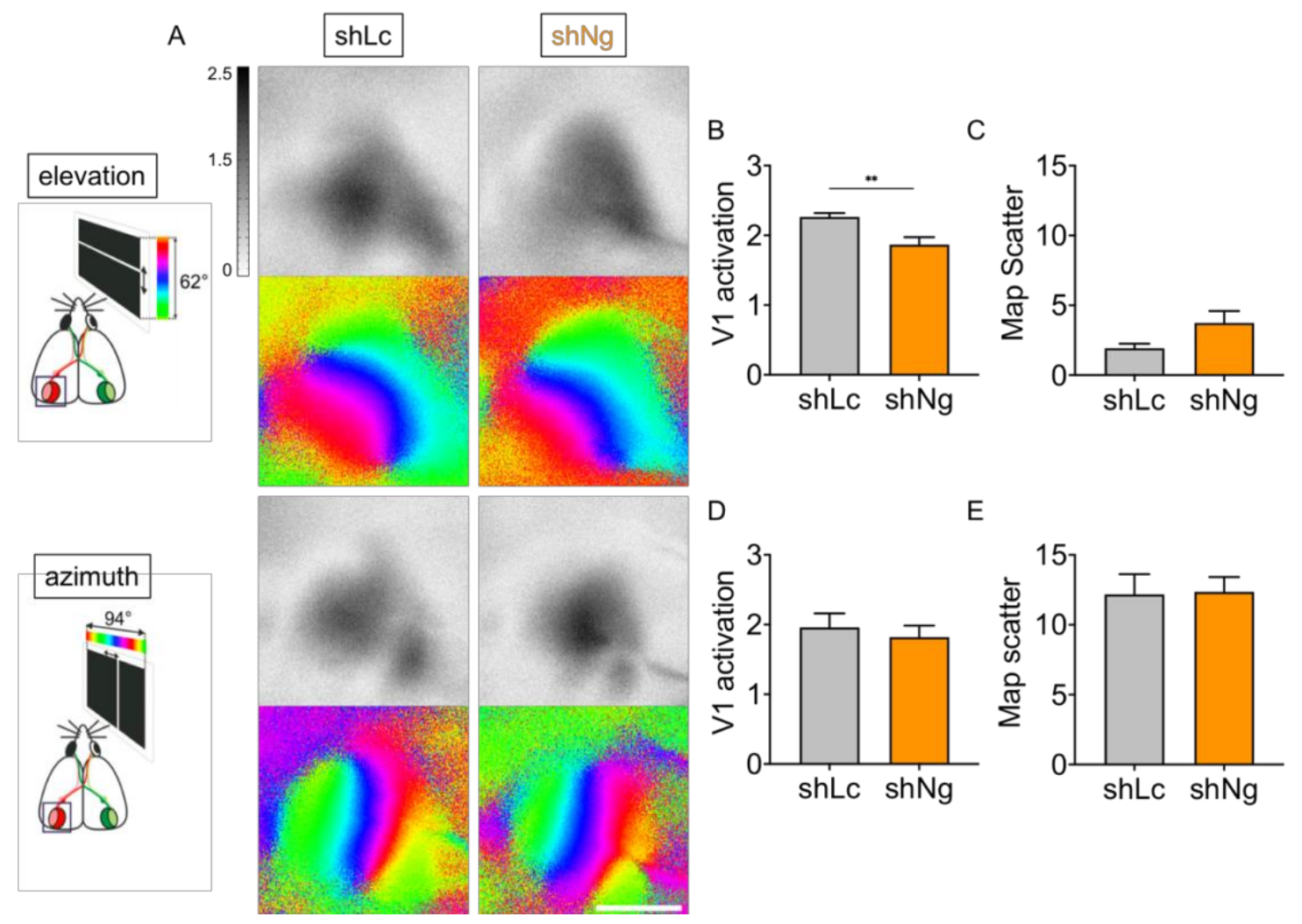

Figure 3. Normal retinotopic maps in shLc and shNg transduced mice. (A) Optically recorded activity and retinotopic maps and their quantification (B-E) in the left V1 of $\sim P 60$ shLc and shNg mice. Both elevation and azimuth maps resulting from stimulation of the right eye (contralateral) with moving horizontal or vertical bars are illustrated. Gray-scale coded response magnitude maps (upper) and retinotopic color-coded phase maps (lower) are shown (scale bar, $2 \mathrm{~mm}$.). Retinotopic maps are color-coded according to the position of the stimulus on the monitor that activated the cortical area (see schematics). (B), (D) Quantification of $\mathrm{V} 1$ activation, expressed as fractional change in reflection $\times 10^{-4}$, and map quality/scatter for (C) elevation, and (E) azimuth maps are plotted. ${ }^{* *} p<0.01$ 


\section{Visual acuity of neurogranin knockdown mice is impaired.}

It has been previously shown that the loss of neurogranin in the visual cortex leads to impaired visually guided behavior in the visual cliff test (Han et al., 2017); however, the acuity of their vision was not tested. Here we tested the maximal visual acuity of shNg mice using the visual water task (VWT), a visual discrimination task based on reinforcement learning (Prusky et al., 2000). Mice ( P45) were trained to swim toward the rewarded monitor displaying a sine wave grating, while the monitor with isoluminant gray was the nonrewarded option (Fig. 1A). After mice had learned the task, the spatial frequency of the grating was stepwise increased to test their visual acuity limit (Fig. 1B). Three consecutive
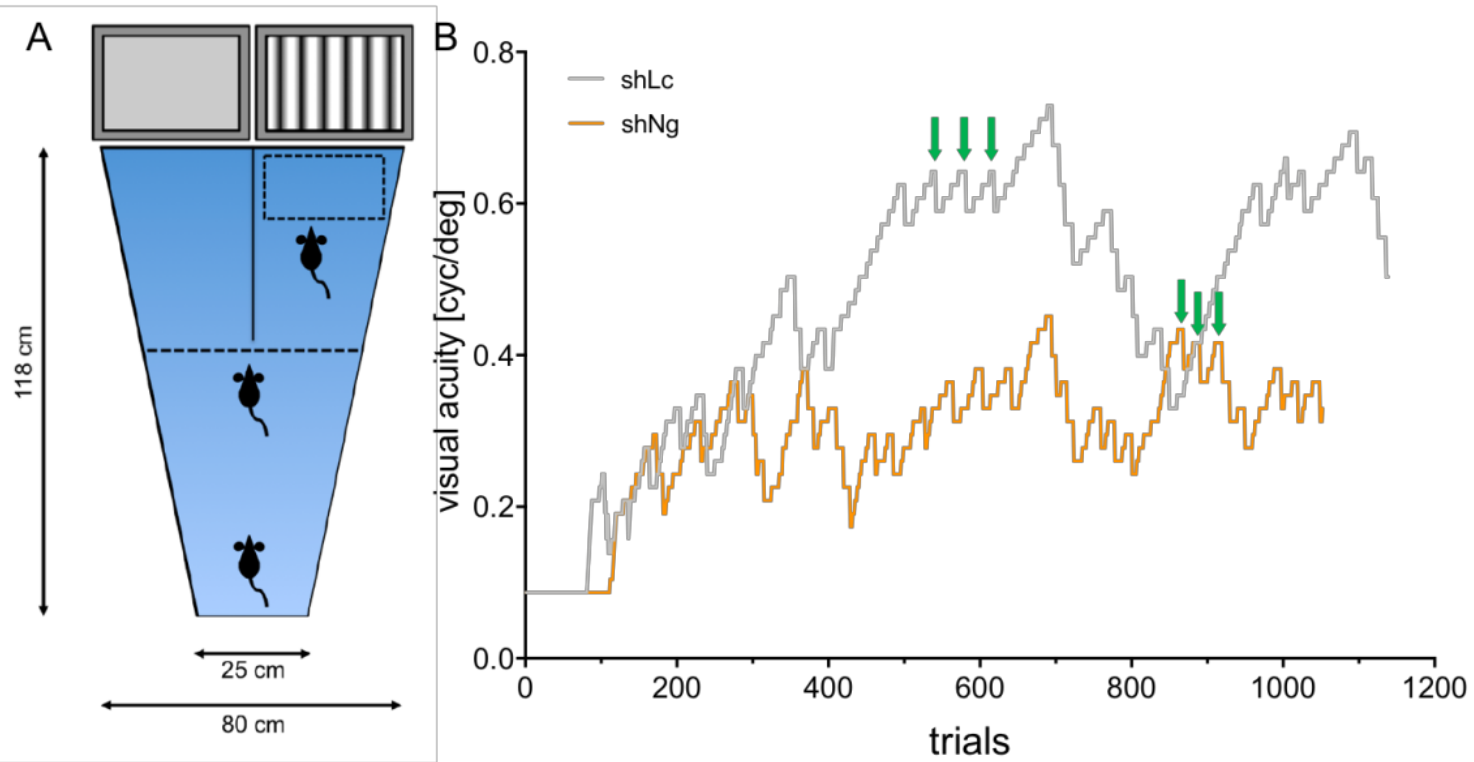

C

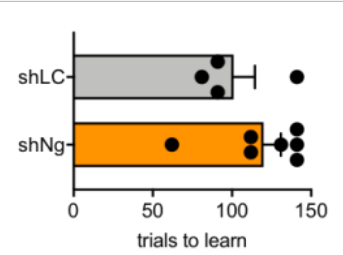

D

\section{E}

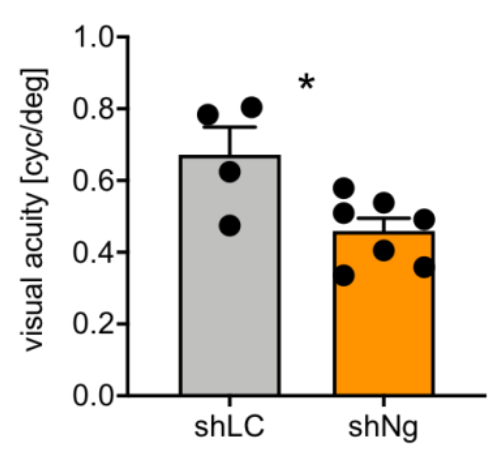

Figure 4. Visual acuity of shNg mice is impaired. Scheme of the VWT setup (A) showing the water-filled trapezoid pool, the midline divider, choice line, submerged escape platform, and the monitors on which visual stimuli are projected. Initially, mice were trained to swim towards the sinusoidal vertical grating (rewarded escape platform) at $0.087 \mathrm{cyc} / \mathrm{deg}$. For 
measuring the visual acuity, spatial frequency of the grating was stepwise increased until the success rate in swimming towards rewarded stimulus fell below $70 \%$ in 3 consecutive blocks of ten trials. (B) Stepwise increase in spatial frequency (cyc/deg) during the testing of two mice plotted against total trials of VWT. Green arrows depict the three consecutive failures at the highest point in the visual acuity curve. (C) Number of trials spent in the training phase. (D) Total number of trials in VWT. (E) Visual acuities of individual mice are presented as dots and the mean \pm SEM as the bar plot. * $p<0.05$.

failures to reach $70 \%$ accuracy at similar spatial frequencies ( \pm 1 step $/ 0.017$ cyc/deg) was taken as their limit (Fig. 1B, green arrows), and the spatial frequencies one step below the failures were averaged to calculate the visual acuity. Mice in control $(n=4) \operatorname{or} \operatorname{shNg}(n=7)$ groups needed on average similar number of trials (101 \pm 14 shLc vs. $120 \pm 11$ shNg; twotailed t-test, $p=0.310$ ) to reach $90 \%$ accuracy in the training phase (Fig. $1 \mathrm{C}$ ), and the total number of trials during the testing did not differ significantly between groups (Fig. 1D) $(1111 \pm 33$ shLc vs. $1089 \pm 27 \mathrm{shNg}, p=0.689)$. The maximum visual acuity of shNg mice was determined (Fig. 1E) as $0.460 \pm 0.035 \mathrm{cyc} / \mathrm{deg}$, significantly lower than $0.672 \pm 0.077$ cyc/deg in control mice ( $n=4$; two-tailed t-test, $p=0.0179$ ). This results suggest that the deficiency of neurogranin in the visual cortex leads to impairments in vision due to loss of visual acuity, while the associative learning during the task was not affected .

Increase in MD-dependent optomotor reflexes are intact in neurogranin knockdown mice.

We used the virtual reality optomotor system developed by Prusky et al. (2004) to assess the spatial frequency and the contrast sensitivity threshold of the optomotor reflexes in control and shNg mice. The optomotor reflexes are mediated by subcortical pathways, but the experience-induced improvements in the spatial frequency and contrast thresholds of the open eye during MD are cortex-dependent (Prusky et al., 2006). We have tested whether MD-induced increase in optomotor reflexes could be influenced by the reduced shNg expression in the cortex. Spatial frequency and contrast sensitivity thresholds of the optomotor reflex were measured daily in both control and shNg mice before and during the 4-day MD or no-MD period.

Control ( $n=3, P 49-P 52)$ and $\operatorname{shNg}(n=3, P 52-P 60)$ mice without MD had similar spatial frequency thresholds on day 0 (Fig. 5A) (three-way repeated-measures ANOVA with 
A

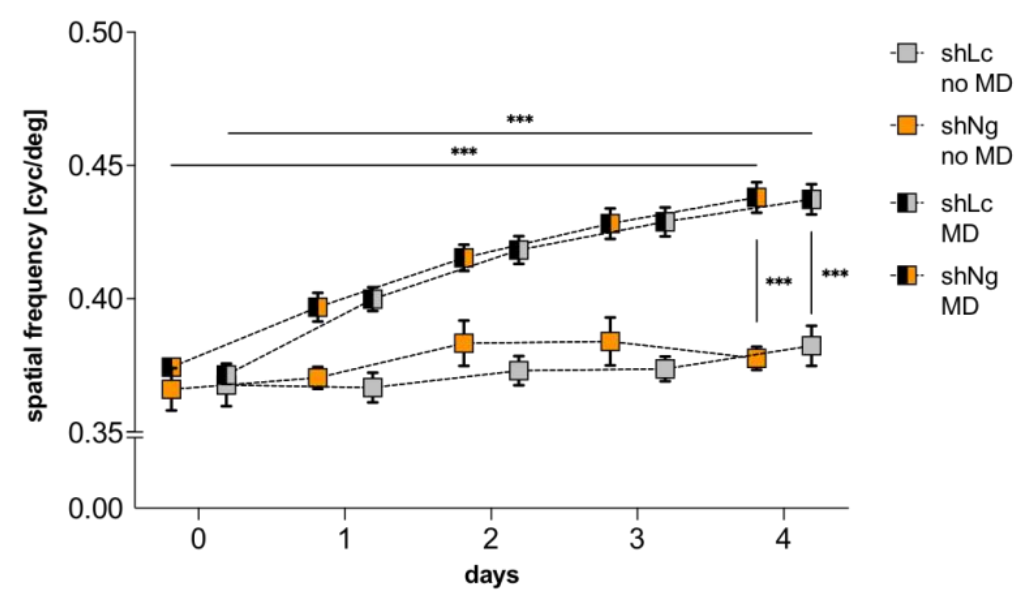

B

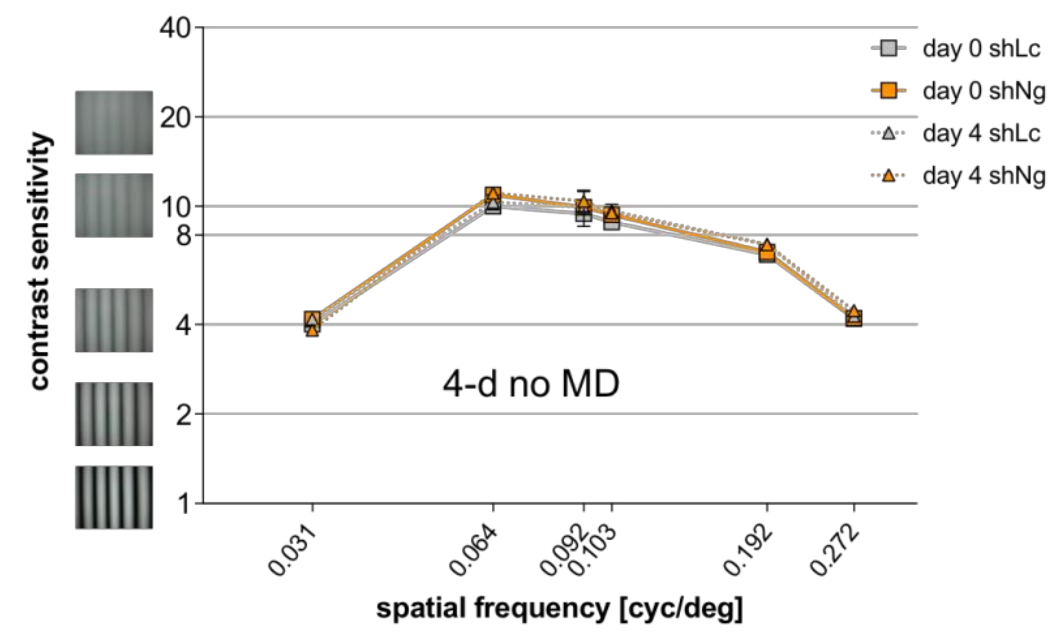

C

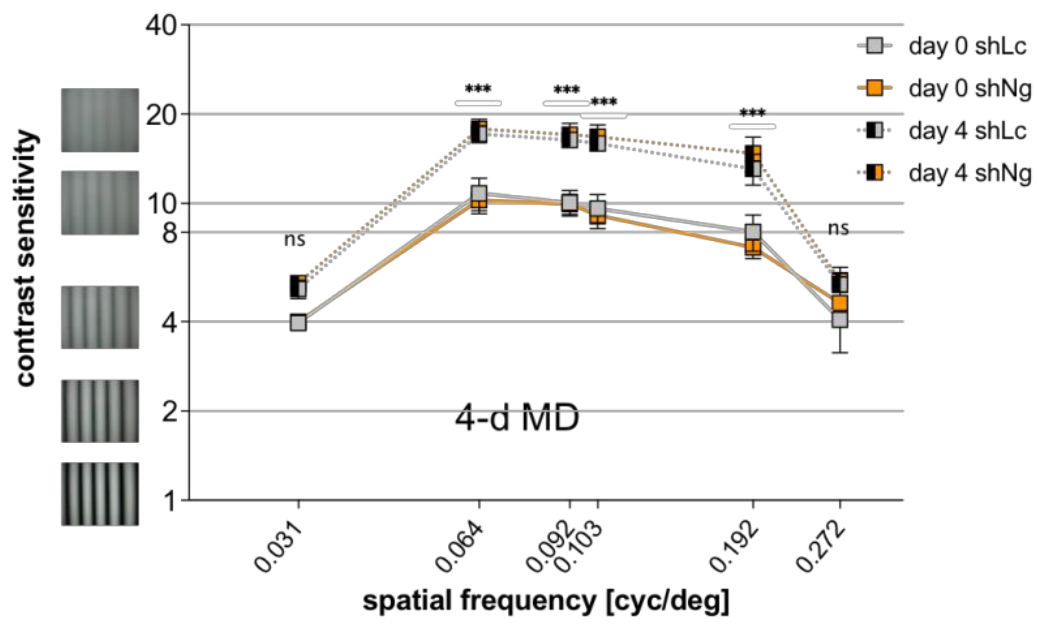

Figure 4. Baseline and experience-enabled improvements of the optomotor reflex threshold. (A) The spatial frequency threshold in cyc/deg of the improvements of the optomotor reflex plotted as a function of days, over $4 \mathrm{~d}$ of MD or no MD. Contrast sensitivity thresholds of the optomotor reflex on days 0 and 4 in mice (B) with no MD and (C) with 4-d MD at six different spatial frequencies. Note that both the spatial frequency and the contrast thresholds increased after MD in both control (shLc) and shNg mice. ${ }^{* * *} p<0.001$ 
Bonferroni adjustment, $p>0.9$ ), and remained stable during $4 \mathrm{~d}$ of testing (control, day 0 : $0.368 \pm 0.008$ cyc/deg vs. day $4: 0.382 \pm 0.008$ cyc/deg, $p>0.9 ;$ shNg, day 0: $0.366 \pm$ $0.008 \mathrm{cyc} / \mathrm{deg}$ vs. day 4: $0.378 \pm 0.004 \mathrm{cyc} / \mathrm{deg})$. In control mice with 4 days of $\mathrm{MD}(\mathrm{n}=7$, P54-P59) the spatial frequency threshold increased from $0.371 \pm 0.008$ cyc/deg to $0.437 \pm 0.006(p<0.001)$. Similar increase was also observed in shNg mice $(n=6$, P56P73) from day $0(0.374 \pm 0.003)$ to day $4(0.438 \pm 0.006)$, thus the spatial frequency thresholds of control and shNg mice were nearly identical after 4-d MD ( $p>0.9)$ (Fig. 5A).

Baseline contrast sensitivity thresholds of control and shNg mice for optomotor reflex were comparable in all tested spatial frequencies (Supplementary Table1) (three-way repeatedmeasures ANOVA with Bonferroni adjustment, $p>0.9$ ). 4-d MD led to comparable improvements in the contrast sensitivity of control and shNg mice at spatial frequencies of $0.064,0.092,0.103$ and $0.172 \mathrm{cyc} / \mathrm{deg}(p<0.001)$, while in mice without MD no significant change was observed over the same period $(p>0.9)$ for the listed frequencies (Fig. 5B,C). After 4-d MD, the contrast sensitivity thresholds of control and shNg mice were not different for any of the spatial frequencies $(p>0.9)$ (Fig. $5 \mathrm{C})$.

These results in summary show that 1) control and shNg mice have normal baseline spatial frequency or contrast sensitivity thresholds for optokinetic reflexes comparable to values reported in previous reports (Greifzu et al., 2016; Hosang et al., 2018; Kalogeraki et al., 2019; Prusky et al., 2004; Prusky et al., 2006) and 2) MD-induced increase in optokinetic reflex thresholds happen independent of neurogranin expression in the cortex . 


\subsection{Discussion}

Here we investigated the effect of virus-mediated reduction of neurogranin expression in critical period (CP) closure and visual capabilities of mice. We demonstrate for the first time that neurogranin, a protein tightly coupled to molecular cascades of synaptic plasticity (Gaertner et al., 2004; Han et al., 2017; Hwang et al., 2018; Petersen and Gerges, 2015) is a regulator of $\mathrm{CP}$ closure for ODP. Silencing the neurogranin expression in V1 preserved enhanced ODP into adulthood even in mice aged up to P149, as evidenced by reduced deprived eye responses after MD, similar to what has been documented in juvenile mice (Espinosa and Stryker, 2012). Moreover, the visual acuity as measured by the VWT was impaired in neurogranin knockdown mice, whereas the visual reflexes measured by visual optomotor test were intact.

Results from the current study support previous findings, where it has been shown that there is a two-way correlation between the course of AMPAR-silent synapse maturation and the duration of the CP for ODP (Favaro et al., 2018). Postsynaptic density proteins (PSD), PSD-95 and PSD-93 are two paralogs that play opposing roles in instructing the AMPARsilent synapse maturation: PSD-95 promotes, while PSD-93 inhibits the maturation of AMPAR-silent synapses (Favaro et al., 2018). The loss of PSD-95 leads to elevated AMPAR-silent synapses and lifelong preservation of juvenile plasticity (Huang et al., 2015), and conversely, the loss PSD-93 results in early decline of AMPAR-silent synapses and precocious closure of the CP for ODP (Favaro et al., 2018). Although, in contrast to neurogranin, PSD-95 and -93 are scaffolding proteins and do not directly control $\mathrm{Ca}^{2+}$ mediated signaling, they are closely implicated in LTP/LTD (Béïque et al., 2006; Carlisle et al., 2008; Schlüter et al., 2006). Despite being part of different molecular apparatuses in the post-synapse, the functions of neurogranin and the PSD-93/-95 paralogs converge in converting AMPAR-silent synapses to AMPAR-transmitting synapses. Thus, removing either one of these molecules has a drastic impact on AMPAR-silent synapses and cortical plasticity as measured by the ODP. Further studies need to confirm how the functions of PSD-95/PSD-93 and neurogranin are interconnected in the molecular chain of events leading to AMPAR-silent synapse maturation. Interestingly, plastic changes induced by traumatic brain injury leads to a correlated decrease in expression levels of neurogranin and PSD-95 in the cortex (Svirsky et al., 2020), suggesting that both are involved in the circuit rewiring. One could postulate that the function of neurogranin is upstream to PSD95/PSD-93, as it is tightly coupled to the early activity-dependent events in the post-synapse that eventually lead to the changes in AMPAR dynamics, which in turn depend on PSD-93/95-mediated receptor trafficking and stabilization. It also needs to be confirmed that the 
fraction of AMPAR-silent synapses in neurogranin deficient mice remains elevated until the timepoint we have used for the optical imaging experiments.

Furthermore, loss of neurogranin led to an impairment in visual acuity of adult mice at the visual water task. This is different from the normal visual acuity of adult PSD-95 KO mice (Favaro et al., 2018; Huang et al., 2015), wherein the fraction of AMPAR-silent synapses is similarly elevated. Notably, loss of neurogranin also leads to exacerbated dendritic spine elimination during development (Han et al., 2017), which is not the case in PSD-95 KO mice (data not shown), revealing differences in the roles played by these two molecules in neural wiring. Our results from the VWT are in line with previous reports, where it has been shown that neurogranin knockout mice have deficits in visually guided behavior in the cliff test (Han et al., 2017), and spatial learning impairment in the Morris water maze (Pak et al., 2000). We have additionally tested the neurogranin knockdown mice in the virtual reality optomotor test (Prusky et al., 2004). Optomotor responses are mediated by subcortical pathways (Giolli et al., 2006); however, the MD-induced potentiation of the reflexes is dependent on cortical projections of V1 to brainstem accessory optic system (Liu et al., 2016; Prusky et al., 2004). Silencing neurogranin expression in the cortex did not affect the baseline spatial frequency threshold or contrast sensitivity of mice, and the MD-induced improvements of the optomotor reflexes were comparable to control mice. Overall, outcomes of the behavioral tests show that neurogranin is important in V1 for achieving the optimal visual acuity, and the function of cortico-fugal projections are not affected by the loss of neurogranin as evidenced by results of the optomotor test.

In conclusion, these results further consolidate the idea that the experience-dependent silent synapse maturation serves as the mechanism for circuit maturation during developmental critical periods. Loss of neurogranin prevents the closure of the $\mathrm{CP}$, and preserves the juvenile-like cortical plasticity into adulthood. Further studies should be directed to understanding whether manipulating neurogranin expression acutely during adulthood can also enhance the cortical plasticity. Knowing this could potentially open new therapeutic avenues in treating diseases such as stroke, wherein enhancing brain plasticity is of great interest to boost rehabilitation. 


\section{Acknowledgements}

We thank M. Schink for excellent animal care. This work was supported by the German Research Foundation through the Collaborative Research Center 889 "Cellular Mechanisms of Sensory Processing" [to S.L. (Project B5) and O.M.S. (Project B3)] and by the Federal Ministry of Education and Research, Germany (Grant 01GQ0810 to S.L.).

\section{Author Contributions}

Study design by R.Y., S.L. and O.M.S. Data collection by R.Y. Data analysis by R.Y. Data interpretation by R.Y., S.L., and O.M.S. Manuscript preparation by R.Y.

\section{Competing interests}

The authors declare no competing interests.

\section{Correspondence}

sloewel@gwdg.de 


\subsection{Supplementary Information}

\section{Supplementary Figure 1.}
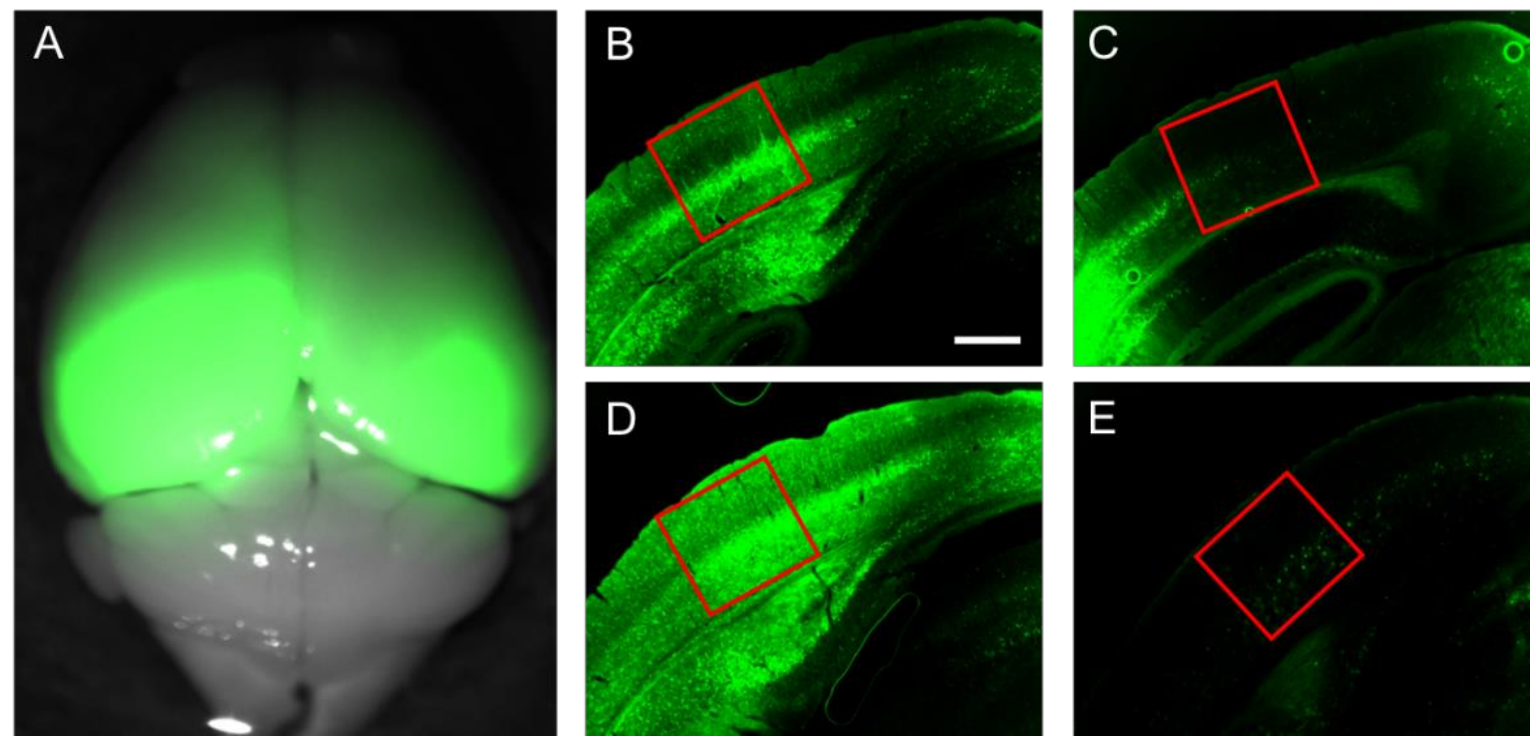

Figure S1. Confirmation of successful transduction in AAV-injected mice. (A) Location of AAV expression (green channel) in P0 V1-injected whole brain (gray). (B-E) Fluorescent images of coronal slices (thickness: $50 \mu \mathrm{m}$ ) from mice brains injected with GFP expressing shLc (B) and shNg AAVs. (D) Binocular V1 is denoted by a red square. Mice without sufficient GFP expression in binocular V1 (C,E) were excluded from analyses. Scale bar, $500 \mu \mathrm{m}$. 


\section{Supplementary Table 1.}

\begin{tabular}{|c|c|c|c|c|}
\hline & $\begin{array}{c}\text { shLc / no MD } \\
(n=3)\end{array}$ & $\begin{array}{c}\text { shLc/4d MD } \\
(n=7)\end{array}$ & $\begin{array}{c}\mathrm{shNg} / \mathrm{no} \mathrm{MD} \\
(\mathrm{n}=3)\end{array}$ & $\begin{array}{c}\text { shNG / 4d MD } \\
(n=6)\end{array}$ \\
\hline $\begin{array}{c}\text { Spatial frequency } \\
\text { (cyc/deg) }\end{array}$ & \multicolumn{4}{|c|}{ day 0} \\
\hline 0.031 & $4.011 \pm 0.137$ & $3.957 \pm 0.036$ & $4.169 \pm 0.169$ & $4.001 \pm 0.073$ \\
\hline 0.064 & $10.006 \pm 0.316$ & $10.810 \pm 0.511$ & $10.922 \pm 0.182$ & $10.258 \pm 0.414$ \\
\hline 0.092 & $9.438 \pm 0.209$ & $10.063 \pm 0.374$ & $9.922 \pm 0.398$ & $9.955 \pm 0.316$ \\
\hline 0.103 & $8.846 \pm 0.284$ & $9.633 \pm 0.409$ & $9.363 \pm 0.472$ & $9.134 \pm 0.366$ \\
\hline 0.192 & $6.876 \pm 0.068$ & $8.024 \pm 0.425$ & $7.013 \pm 0.389$ & $7.109 \pm 0.239$ \\
\hline 0.272 & $4.190 \pm 0.118$ & $4.056 \pm 0.039$ & $4.202 \pm 0.047$ & $4.619 \pm 0.603$ \\
\hline $\begin{array}{l}\text { Spatial frequency } \\
\text { (cyc/deg) }\end{array}$ & \multicolumn{4}{|c|}{ day 4} \\
\hline 0.031 & $4.172 \pm 0.106$ & $5.149 \pm 0.137$ & $3.838 \pm 0.066$ & $5.391 \pm 0.125$ \\
\hline 0.064 & $10.325 \pm 0.596$ & $17.148 \pm 0.425$ & $11.082 \pm 0.198$ & $17.848 \pm 0.556$ \\
\hline 0.092 & $9.944 \pm 1.377$ & $16.371 \pm 0.305$ & $10.408 \pm 0.804$ & $17.048 \pm 0.650$ \\
\hline 0.103 & $9.661 \pm 0.492$ & $15.933 \pm 0.302$ & $9.516 \pm 0.361$ & $16.778 \pm 0.668$ \\
\hline 0.192 & $7.446 \pm 0.255$ & $13.088 \pm 0.588$ & $7.444 \pm 0.236$ & $14.747 \pm 0.809$ \\
\hline 0.272 & $4.267 \pm 0.062$ & $5.341 \pm 0.126$ & $4.456 \pm 0.082$ & $5.487 \pm 0.149$ \\
\hline
\end{tabular}

Table S1. Contrast sensitivity thresholds for optomotor reflex. Average contrast sensitivity thresholds for all the tested spatial frequencies in each group on day 0 (upper) and day 4 (lower) are listed in the table as mean \pm SEM. 


\section{General Discussion}

The results presented in this thesis advance our knowledge in understanding the molecular and structural mechanisms of cortical plasticity in the context of ocular dominance plasticity (ODP) in several important ways outlined below:

1) In adult mice, postsynaptic density protein (PSD)-95 limits the experiencedependent structural dynamics of dendritic spines on the apical dendrites of layer (L) 2/3 pyramidal neurons (PNs) of the primary visual cortex (V1).

2) Increased spine elimination in apical dendrites of $L 2 / 3$ PNs of the binocular $V 1$ may be an anatomical substrate for decreased deprived eye responses in juvenile-like ODP.

3) Spine dynamics during recovery from MD (i.e. amblyopia) are not mediated by reversal of MD-induced perturbations, suggesting mechanistic differences between recovery from amblyopia and induction of it.

4) ODP can be expressed cell-autonomously in a structural level.

5) Neurogranin, a postsynaptic signaling protein recently described to promote the maturation of AMPAR-silent synapses, is a regulator of critical period closure for ODP.

6) Neurogranin is required for achieving normal visual acuity in adulthood.

I will discuss these findings broadly in relation to previous studies investigating cortical plasticity from both structural and molecular perspectives, while also considering the differences in experimental designs and technical challenges associated with different approaches. As I go through these matters, I intend to mainly address the questions outlined below:

1) How do cells in different layers of sensory cortices get involved in circuit reorganization during experience-dependent plasticity?

2) There is ample evidence in favor of the idea that AMPAR-silent synapses are the developmental substrates for experience-dependent maturation of glutamatergic cortical circuits. How do AMPAR-silent synapses participate in plastic changes induced by sensory manipulations?

3) PSD-95 and neurogranin, both enriched at the postsynaptic membrane of excitatory neurons, have now been closely associated with AMPAR-silent synapse maturation. Are their functions in regulating the maturation of circuits and consequently critical period plasticity similar? 


\subsection{Experience-dependent structural plasticity}

During the critical period for ODP in mice ( P19-P32), 4-d MD induces strong ODP, mediated via reduction of cortical responses to deprived eye stimulation, a hallmark of juvenile ODP (Espinosa and Stryker, 2012). In mice reared with standard housing conditions, juvenile-like ODP is lost after the closure of critical period (>P35) (Gordon and Stryker, 1996). Recently, experience dependent maturation of AMPAR-silent synapses was shown to mediate the critical period closure in mice V1 (Favaro et al., 2018; Huang et al., 2015). In the visual cortex of adult PSD-95 KO mice, $~ 50 \%$ of synapses in the L4-to-L2/3 PN circuitry are AMPAR-silent. Abundance of AMPAR-silent synapses conferred adult (up to P480) PSD-95 KO mice juvenile-like ODP: 4-d MD led to a drastic decrease in deprived eye responsivity in binocular V1 (Huang et al., 2015). In the present study, I show that, in adult PSD-95 KO ( P75) mice, 4-day MD of the contralateral eye led to a significant increase $(\sim 130 \%)$ in spine elimination rate and a significant decrease in spine gain $(60 \%)$, at the apical dendrites of L2/3 PNs of binocular V1. In PSD-95 WT mice, however, spine dynamics during 4-d MD, compared to the baseline, remained essentially unchanged. Increase in spine elimination was significantly higher in PSD-95 KO mice compared to WT controls, but the difference in spine formation rates during 4-d MD did not reach significance between the genotypes. MD-induced perturbations in the spine dynamics led to a significant $(\sim 6 \%)$ decrease in the spine density in PSD-95 KO dendrites, whereas spine density in WT mice did not change significantly during the course of imaging. Moreover, MD led to similar increase in spine elimination rates in the dendrites of single neurons in which PSD-95 expression was silenced. These data suggest that the increased spine elimination in adult PSD-95-deficient mice is the anatomical correlate of the reduction in deprived eye responses following 4-d MD.

In the following sections, I will survey previous studies which have investigated the spine dynamics in the context of ODP. As I have only investigated the spine dynamics in L2/3 $\mathrm{PNs}$ of binocular $\mathrm{V} 1$, it is essential to consider the findings from different cortical lamina, in order understand the full scale of plastic changes happening in association with ODP. I will also refer to studies done in the barrel cortex of rodents. Surveying the commonalities and differences in circuit remodeling associated with manipulations such as ODP paradigm and whisker trimming may paint a more thorough picture of cortical plasticity. Many of the studies I will discuss here have been reviewed before (Berry and Nedivi, 2016, 2017; Fu and Zuo, 2011; Holtmaat et al., 2009; Holtmaat and Svoboda, 2009; Moyer and Zuo, 2018), but the cortical layer-, age-, and modality-specific plastic changes in response to different sensory manipulations had not been discussed systematically. 
Notably, the studies I will mention here have used anesthetized animals for investigating the spine dynamics, while I have performed all the spine imaging experiments in awake mice in order to exclude potential anesthesia effects. Previous evidence suggests that exposure to anesthetics can influence spine dynamics. In rat hippocampal slices, in-vitro exposure to $2 \%$ isoflurane (in oxygen), a commonly used volatile anesthetic, causes spine loss within first 10 minutes of exposure and induces spine shrinkage after 60 min (Platholi et al., 2014). In another study with rat hippocampal cell cultures, exposure to dissolved isoflurane, at a dose clinically used for general anesthesia $(0.3 \mathrm{mM})$, was shown to decrease the spine motility (Kaech et al., 1999). In the prefrontal cortex of P15-16 rats, 1-hour exposure to $1.5 \%$ isoflurane increased the spine density at basal and apical dendrites of layer 5 PNs (Briner et al., 2010). In one-month-old mice, single 4-hour exposure to 1.5\% isoflurane decreased filopodia elimination in vivo in apical dendrites of layer 5 PNs in the barrel cortex, although the spine dynamics were unaffected (Yang et al., 2011). 5-hour anesthesia with midazolam or ketamine, increased spine formation in the apical dendrites of L5 PNs in somatosensory cortex and in CA1 PNs in hippocampus of (P15-30) YFPexpressing mice (De Roo et al., 2009). Although the effects of anesthesia have not been studied in the visual cortex of adult WT or PSD-95-deficient mice, previous studies suggest that anesthesia can interfere with spine dynamics.

\section{Experience-dependent dynamics in layer 4 neurons}

Although dendritic spines of (L) 4 PNs in V1 have not been investigated using longitudinal two-photon imaging in living animals, several studies in the late $90 \mathrm{~s}$, before the advent of two-photon microscopy, have focused on this layer to characterize its relevance in ODP both in cats and mice (Antonini et al., 1999; Silver and Stryker, 1999). L4 received the early attention partly because thalamocortical synapses are the first entry point for sensory signals to reach the cortex. Most of the thalamic input is received in L4 of the cortex via spiny stellate neurons, which in turn by sending vertical axonal branches synapse onto L2/3 PNs as shown in the visual cortex of cats (Gilbert, 1983), macaque monkeys (Callaway, 1998), and the barrel cortex or rats (Feldmeyer et al., 2002). Interestingly, however, a series of experiments discovered that thalamocortical connections in L4 initially show only limited, if at all, anatomical plasticity in response to brief MD. Although the effect of MD on deprived eye responses is much more rapid and saturates within 2-3 days in [cats (Hubel and Wiesel, 1970), mice (Dräger, 1978)] remodeling of thalamocortical afferent arbors in juvenile animals takes place over days to weeks after a prolonged MD in cats (Antonini and Stryker, 1993) and mice (Antonini et al., 1999). It was then hypothesized that the rapid reduction in deprived eye responses after MD must be due to the elimination of geniculo-cortical 
synapses in L4 (Silver and Stryker, 1999). However, this hypothesis was rejected after it was found that in cats, L4 geniculo-cortical synapses in V1 remain surprisingly stable during a brief 2-d MD (Silver and Stryker, 1999). Using optical imaging of intrinsic signals, it was later shown that, in cats, cortical responses to deprived eye diminish already 24 hours after the initiation of MD (Trachtenberg et al., 2000). Performing microelectrode penetrations in the same animals, single cell responses in binocular $\mathrm{V} 1$ revealed that cells above and below L4 lose responsivity to deprived eye stimulation after 24-hour MD, whereas L4 cells still show normal binocular responses (Trachtenberg et al., 2000). These results provided an explanation for the predominating anatomical observations that geniculo-cortical synapses do not show rapid structural plasticity (Silver and Stryker, 1999); and that changes in extragranular layers happen much faster than at the input layer (in $24 \mathrm{~h}$ versus $7 \mathrm{~d}$ in L4) (Antonini and Stryker, 1993). Consistent with the aforementioned observations, in vivo imaging of population of presynaptic boutons in the barrel cortex showed that $\sim 85 \%$ of thalamocortical afferents persisted throughout all imaging sessions over a 1-month period, whereas the cortico-cortical boutons were more transient and a much lower proportion ( 40-70\%) persisted during the same period. (De Paola et al., 2006). In vivo spine dynamics on L4 PNs in the barrel cortex of adult mice, however, seem to show rapid plastic changes (4day) in response unilateral whisker trimming, where the apical dendrites of L4 PNs undergo increased ( 50\%) spine elimination (Miquelajauregui et al., 2015).

\section{Layer $2 / 3$}

In-vivo two-photon imaging studies of spine dynamics in L2/3 PNs have been scarce in comparison to studies investigating apical dendrites of L5 PNs. This is partly due to difficulties in selectively labeling the L2/3 cells, while studying L5 PNs has been relatively easy due to the availability of a transgenic mouse line, in which the thy 1 promoter drives the expression of GFP (or YFP) in a subset of mainly L5 PNs (Feng et al., 2000). On the other hand, studying spines in L2/3 PNs is possible largely by using the in-utero electroporation technique (Saito, 2006; Saito and Nakatsuji, 2001). This technique takes advantage of the fact that the birth of the precursors cells for each cortical layer happens successively on different time points approximately between the embryonic days $(E) 13.5$ E16.5 in mice (Angevine and Sidman, 1961). Electroporating developing cortex of mouse pups at E15.5 transfects the progenitors of layer 2/3 cells, which enables not only fluorescently labeling them, but also opens up the possibilities to do layer-specific modifications in gene expression. To achieve transfection in the cortical progenitor cells, plasmid DNA is delivered to the lumen of ventricular system via a microinjection, after which the head of the embryo is held between two oppositely charged plates and an electric pulse 
is applied. The axis of the applied pulse targets a certain neurogenic area in the ventricular zone, giving rise to expression in the cortical area that develops from the transfected neurogenic zone (Saito and Nakatsuji, 2001). A noteworthy technical issue, however, is reliably electroporating the visual cortex, due to limited physical access for positioning the electrodes to target the caudal cortical areas in embryos. Thus, there are only a handful of papers which have reported using in-utero electroporation to label the cells in the visual cortex for in-vivo imaging of spine dynamics (Cane et al., 2014; Chen et al., 2012; Sun et al., 2019). I had $\sim 10-30 \%$ success rate in achieving GFP expression in binocular V1. In most cases, the foci of expression was lateral to binocular $\mathrm{V} 1$, mainly targeting $\mathrm{V} 2$ and the auditory cortex (see Fig. D1).

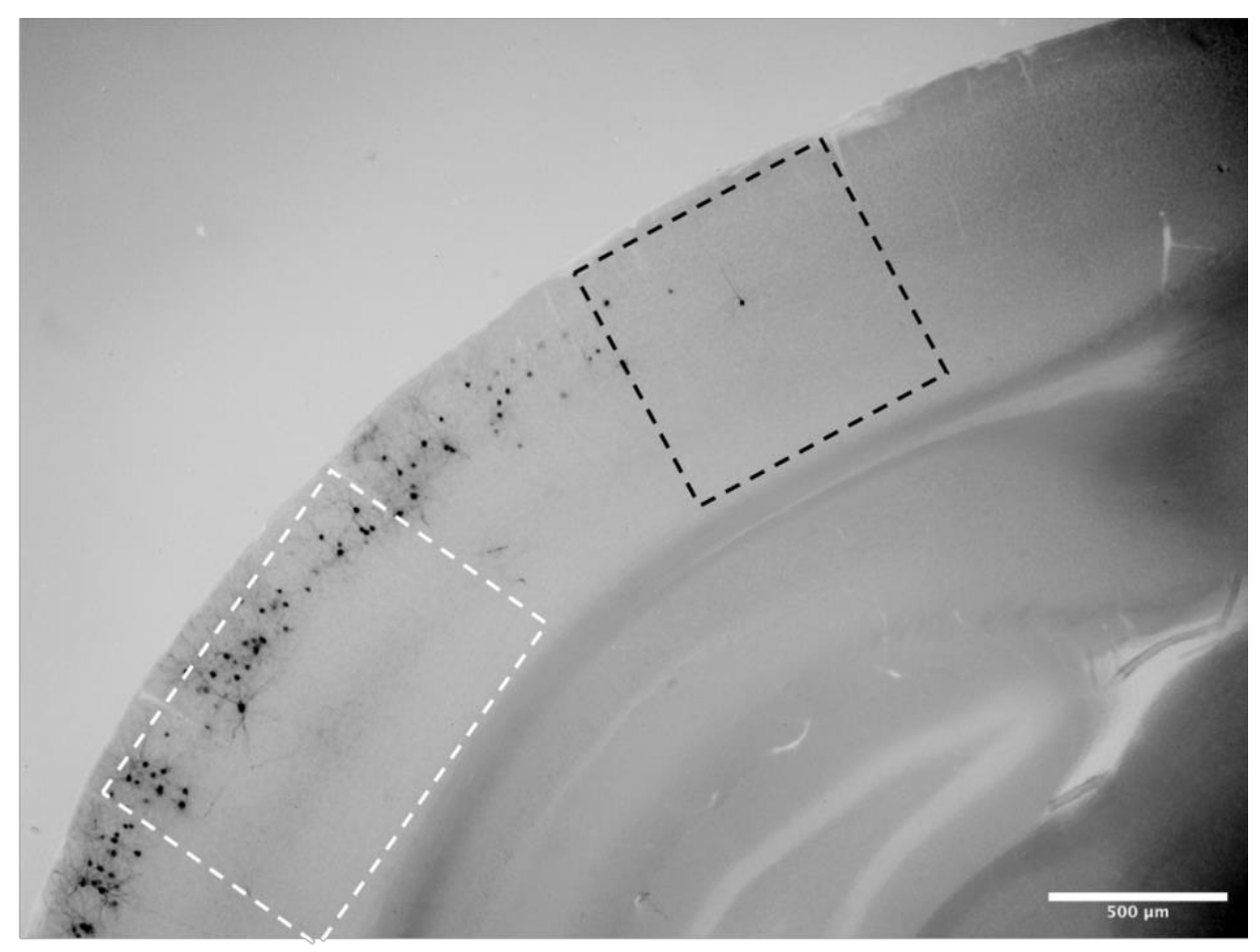

Figure D1. Coronal brain slice (AP: -3.16) of an E15.5 in-utero electroporated mouse brain perfused at P71. Targeting V1 with in-utero electroporation often led to an off-site expression of the construct in nearby sensory areas, mainly the auditory cortex. Dashed rectangles depict the location of binocular V1 (black) an auditory cortex (white). In this case most of the GFP-expressing neurons were in the auditory cortex. Black and white pixels of the image are inverted for improving the visibility of GFP expressing cells. Slice thickness, $200 \mu \mathrm{m}$. 
By labeling the L2/3 PNs of the binocular V1 via delivery of lipophilic Dil-coated tungsten or gold beads into fixed mouse brain slices, one of the earlier studies have shown that during the critical period for ODP, 4-d MD causes a reduction in spine density ( $10-40 \%$ compared to no MD condition) in the apical dendrites (Mataga et al., 2004). Moreover, during the critical period for ODP, a brief MD (2-3 days) rapidly diminishes the L2/3 PN responses to deprived eye stimulation, as evidenced by calcium imaging experiments, where binocular V1 of mice were imaged after bulk loading the cortex with a cell-permeable calcium indicator dye (Oregon Green BAPTA-1) (Mrsic-Flogel et al., 2007). Consistent with aforementioned studies, Sun et al. (2019) recently demonstrated that in juvenile mice (P28-36) 3-d MD nearly doubles the rate of postsynaptic puncta elimination ( $97 \%$ increase) when compared to mice which did not receive MD over the same period, and results in net $\sim 8.7 \%$ decrease in postsynaptic puncta after 3-d MD. These values are similar to the results presented here in adult PSD-95 KO mice (P64-P79), where I have observed an average $\sim 130 \%$ increase in spine elimination after MD in apical L2/3 dendrites compared to baseline, which led to a $\sim 6 \%$ decrease in spine density during MD. Nevertheless, it has to be mentioned that, in this study, postsynaptic compartments were visualized by expressing GFP tagged PSD-95. Such overexpression of PSD-95 may have stabilized the spines, although authors did not detect any impact of PSD-95-GFP on ODP of juvenile mice (P28) even when the expression of PSD-95-GFP was increased 500-fold (Sun et al., 2019).

Moreover, Sun et al. (2019) study established a direct correlation between spine elimination and the OD-shifts: dendrites that were dominated by the contralateral input lost more puncta during the deprivation. Strong correlation between functional OD plasticity and MDdependent spine pruning has also been observed in L2/3 PN apical dendrites in the visual cortex of ferrets (Yu et al., 2011), although in this study they used virus injections for labeling the dendrites, which could have transfected the neurons in deeper layers as well. Such correlations between MD-induced spine loss and OD shifts of individual dendrites are important to ascertain the magnitude of experience-dependent circuit rewiring for the following reason. In adult mouse V1, not all of the neurons in layer 2/3 exhibit contralateral dominance (although the majority does), and only $\sim 60 \%$ of those undergo OD shifts after MD (Rose et al., 2016). Considering this, during spine imaging it is very probable to encounter dendrites which either do not receive input dominated by the contralaterally eye to begin with or alternatively do not show detectable OD-shifts after 5-8 days of MD (Rose et al., 2016). In population studies of spine dynamics in binocular V1, blind pooling of dendrites with various OD values underestimates the structural remodeling due to eyespecific changes. More specifically, dendrites receiving input dominated by the contralateral eye undergo greater plastic changes in response to contralateral eye deprivation than 
dendrites receiving ipsilaterally dominated input (Sun et al., 2019). Thus, averaging the plastic changes across dendrites with different inputs may mask the extent of plastic rewiring in response to deprivation. This becomes an especially pressing issue during awake imaging compared to anesthetized, where the sample sizes per animal are low due to time constraints. Simultaneously imaging the ocular dominance of both pre- and postsynaptic compartments in conjunction with tracking the spines longitudinally would give us a much clearer picture of plastic changes following the visual deprivation. Nevertheless, such an experiment is technically challenging in that it requires 1) pre- and post-synaptic structural labeling and expression of a calcium indicator that would allow monitoring both morphological changes and calcium signals at the same time, and 2) faster scanning speeds at the two-photon microscope to visualize calcium activity in response to eye stimulation at the synaptic level.

In adult mouse brain, L2/3 spine dynamics on the apical dendrites are not affected even after 4- or 8-d MD (Hofer et al., 2009), which is consistent with the results shown in this thesis: during 4-d MD spine dynamics in apical dendrites of L2/3 PNs in adult PSD-95 WT mice remained stable. Notably, in vivo dynamics of inhibitory synapses in L2/3 of V1 has been shown to increase during MD in adult mice (Chen et al., 2012; Villa et al., 2016). In the barrel cortex of rats, trimming all the whiskers unilaterally for 2 days (P11-13) during the critical period (P5-P13) does not affect the spine density in L2/3 apical dendrites (Lendvai et al., 2000). Prolonged period of whisker trimming (7-d) reduces the rate of spine elimination, while not affecting spine formation in the apical dendrites of L2/3 PNs in the barrel cortex of 1 month-old mice (Ma et al., 2016). Removing every other whisker in a chessboard pattern, which might be more similar to MD, in adult mice increases spine formation in the basal dendrites of L2/3 PNs, while not affecting the apical dendrites (Seaton et al., 2020). Unfortunately, so far very small number of studies focusing on the dendritic spines of L2/3 PNs makes it hard to draw conclusions between visual and barrel cortices, but it seems that in adulthood apical dendrites of L2/3 PNs in both cortices are less malleable by sensory deprivations.

\section{Layer 5}

In binocular V1, during the critical period for ODP (P28), closing the contralateral eye for 3 days leads to increased ( $73 \%$ ) spine elimination in apical dendrites of L5 PNs (Zhou et al., 2017), comparable to what has been reported for apical dendrites of $L 2 / 3$ PNs (Sun et al., 2019). In adult mice (P45-100), however, 4 day MD elevates the spine gain rate ( $80-100 \%)$ in the apical dendrites of L5 PNs and leads to an increase ( 8\%) in spine density (Hofer et 
al., 2009). Similarly, in juvenile mice MD initially reduces the deprived eye responses after 2-3 days (Frenkel and Bear, 2004), while in adults OD shifts are mediated by increase in open eye responses (Sato and Stryker, 2008; Sawtell et al., 2003). While the reduction in deprived eye responses in juvenile mice after brief (2-3d) MD correlate with increased spine elimination in the apical dendrites of L2/3 and L5 PNs (Frenkel and Bear, 2004; Sun et al., 2019; Zhou et al., 2017), in adulthood, open eye potentiation detected between 5-8 d of MD correlates with increased spine gain in the apical dendrites of L5 PNs in a similar time frame (Sato and Stryker, 2008; Sawtell et al., 2003). Thus, there seems to be a two-way correlation between functional and structural plasticity, which needs to be confirmed in further studies. Based on these findings, one can postulate that newly added spines in response to MD of the contralateral eye primarily receive ipsilateral, open eye input and thus contribute to increase the overall responsiveness of the postsynaptic neuron to ipsilateral eye stimulation.

In the barrel cortex of young adult mice (P42-70), chessboard whisker trimming does not change the spine density at the apical dendrites of L5 PNs, but leads to increased spine $(\sim 50 \%)$ turnover rates after $2-4$ days of whisker trimming compared to the pre-deprivation period (Trachtenberg et al., 2002). After 8-20 days, chessboard whisker trimming results in stabilization of newly formed spines while increasing the elimination of previously persistent spines in 2-5 month old adult mice (Holtmaat et al., 2006). Trimming all whiskers on the contralateral side in 1-month old mice, leads to reduced spine elimination ( $50 \%)$ in the apical dendrites of L5 PNs after 2 weeks (Zuo et al., 2005).

In summary, there is ample evidence showing that in V1 loci of rapid cortical plasticity are dendritic spines located beyond the thalamic input layer of the cortex. Connections of neurons located in both supra- and infragranular layers of V1 get rapidly remodeled during ODP via additions or eliminations of spines. The net outcome of such perturbances in spine dynamics, however, vary between cortical lamina depending on the developmental stage (i.e. juvenile vs. adulthood) of the animal. The effect of sensory manipulation on spine dynamics is also modality specific and varies across sensory cortices. Notably, most studies investigating spine dynamics have looked into population of spines without characterizing their inputs. Thus, future studies directed towards understanding the functional consequences of anatomical changes at the physiological and possibly behavioral levels should offer clearer insights into the principles of cortical wiring and its plasticity. 


\subsection{Recovery from monocular deprivation}

In juvenile mice, MD-induced OD shifts are transient and the contralateral dominance of $\mathrm{V} 1$ recovers quickly if the deprived eye is reopened for 2 days (Hofer et al., 2006; Kaneko et al., 2008). In this regard, PSD-95 KO adult mice have been shown to display the same experience-dependent changes as CP mice (recovery within $2 \mathrm{~d}$ ), while in WT adult mice, restoring the OD takes longer (4 days) (Huang et al., 2015). In the apical dendrites of L2/3 $\mathrm{PNs}$ of CP mice, MD increases the spine elimination after 3-d, followed by increased spine formation if the MD is extended to 6-d. A subsequent 2-d recovery from 6-d MD reverts the spine elimination and formation rates to normal (Sun et al., 2019). However, MD-dependent (3-d) increase in spine elimination persists in the apical dendrites of L5 PNs after $2 \mathrm{~d}$ of recovery in CP mice (Zhou et al., 2017). Interestingly, in this study, during the recovery phase spine dynamics of adult PSD-95 KO mice did not differ from age matched WT controls after 2- or 4-days of recovery. Intragroup comparisons in PSD-95 KO group revealed that MD-induced increase in spine elimination persisted after 4-d of reopening, while in WT group there were no significant changes throughout MD or subsequent reopening. A possible explanation for persistently elevated spine elimination in PSD-95 KO mice could be that while MD affects spine populations that are dominated by contralateral eye input, during the subsequent recovery, spines with ipsilateral OD get pruned, thus shifting the $\mathrm{OD}$ of neurons in the contralateral eye direction. Furthermore, recovery of deprived eye responses to normal after the reopening would require increased spine formation or spine enlargement. As I did not observe increased spine formation in PSD-95 $\mathrm{KO}$ mice during recovery, the data presented here can exclude the first of those two scenarios. Thus, recovery of deprived eye responses is most likely achieved by strengthening the previously existing connections possibly by increasing the spine head sizes, rather than making new spines. The latter scenario could indirectly be supported by findings of a previous study, where in adult mice a second MD did not result in new spine formation in the apical dendrites of L5 PNs, but increased the sizes of spines gained during the first MD (Hofer et al., 2009). In order to find a definitive answer to the mechanisms of recovery from $\mathrm{MD}$, it would be essential to image the calcium signals in the pre- and postsynaptic compartments in binocular V1 before, during, and after MD.

\subsection{PSD-95 and dendritic spine stability}

PSD-95 is a major scaffolding protein in the postsynaptic membrane of excitatory synapses that interacts directly with NMDA and indirectly with AMPA receptors (Chen et al., 2000; Kornau et al., 1995). In the visual cortex, PSD-95 protein levels rise after eye opening and reach $\sim 80 \%$ of adult levels around the end of the critical period for ODP (Huang et al., 
2015), an expression profile that has been causatively linked with the developmental decline of AMPAR-silent synapses and CP closure (Favaro et al., 2018; Huang et al., 2015). Notably, in apical dendrites of L5 PNs in the visual cortex of juvenile mice, a larger fraction of spines are morphologically immature at P30 (small head, long necks, $\sim 10 \%$ filopodia) compared to adulthood and turn over more rapidly ( $70 \%$ are persistent, $30 \%$ transient) (Grutzendler et al., 2002). As the animals age (>P42), more of these spines take on mature shapes (shorter neck, bulbous head), filopodia become rare $(<1 \%)$, and the spine dynamics decrease significantly ( $\sim 90 \%$ persistent) (Grutzendler et al., 2002; Holtmaat et al., 2005). Developmental decline of spine dynamics at first sight correlates with the developmental profile of PSD-95 protein level expression, which reaches $80 \%$ of adult levels at P38 (Huang et al., 2015). Although this correlation seems to propose a potential role for PSD-95 in morphological maturation of dendritic protrusions, PSD-95 KO spines in CA1 region of hippocampal slices from rat (P13-16) have similar volume as the WT counterparts, and in PSD-95 mutant mice (that express only the NMDAR binding domains of the protein), spines in CA1 region bear a normal postsynaptic density and are morphologically not different from spines of the wilt-type littermates if investigated using static imaging methods (Béïque et al., 2006; Migaud et al., 1998). Morphological analysis of spines at the basal dendrites of L2/3 PNs in V1 of adult ( P70) mice brains also did not reveal significant differences in spine density, length, or head size between genotypes (data not shown). Thus, gradual transformation from juvenile to mature spine shapes is independent of PSD-95 expression. Nevertheless, in vivo dynamics of PSD-95 clustering in dendritic spines on apical dendrites of L5 PNs in the barrel cortex of P10-21 mice decrease during development and increase in adult >P60 mice in response to clipping of big whiskers on both sides of the snout (Gray et al., 2006). Recruitment of PSD-95 to newly formed spines is activity driven (De Roo et al., 2008; Taft and Turrigiano, 2014). Moreover, in CA1 region of mice hippocampal slice cultures, acute knockdown of PSD-95 by RNA interference (RNAi) increases the dynamics of dendritic spines of PNs measured after 3 days of expression, while it also impairs stabilization of new spines after LTP induction (Ehrlich et al., 2007). In an in-vivo imaging study, it was shown that new spines which do not acquire GFP-tagged PSD-95 puncta are more likely to be lost, whereas the expression of PSD-95-GFP puncta did not impact the baseline spine turnover rates in apical dendrites of layer 2/3 PNs in the barrel cortex of adult >P40 mice (Cane et al., 2014). These findings strongly imply that PSD-95 might play a role in regulating in vivo spine dynamics.

In the present study, during 4-days of the baseline condition, spine dynamics on apical dendrites of L2/3 PNs did not differ significantly between PSD-95 deficient and control mice. During 4-d MD, however, spine dynamics were drastically destabilized in PSD-95 deficient 
mice in favor of increased ( $130 \%$ compared to baseline $)$ spine elimination. Nevertheless, the percentage of persistent spines, with lifetime of $\geq 4$ days, were similar between WT ( $83 \%$ persistent spines) and PSD-95 KO mice ( 82\%) over the periods of normal vision and $\mathrm{MD}(8 \mathrm{~d}$ in total). Interestingly, spines that were newly formed during the baseline condition, were more likely to be eliminated by MD in PSD-95 KO mice: in 30\% of KO dendrites all new spines were lost during 4-d MD, while in WT mice this happened in only $9 \%$ of dendrites.

Notably, previous results in our lab have shown that in adult (>P100) PSD-95 KO mice, baseline spine dynamics at apical dendrites is higher than WT controls (Tippmann, A., 2018, unpublished). In these experiments, dendritic spines in L2/3 of V1 were labelled using virally mediated expression of GFP tagged LifeAct peptide, which stains F-actin in dendritic spines (Riedl et al., 2008). Thus, the imaged dendritic spines could belong to PNs situated in both layers $2 / 3$ and 5 , and were not restricted to supra-granular layers $2 / 3$ like in the present thesis. Layer-specific differences in spine dynamics have not been directly addressed in the binocular V1, but previous studies in the barrel cortex of 3-month old mice showed that spines on apical dendrites of L5 PNs (77\% persistent) are more dynamic compared to L2/3 ( $85 \%$ persistent). In case of similar layer specific differences in $\mathrm{V} 1$, unequal pooling of $L 2 / 3$ versus L5 dendrites in the analyses of WT and KO mice could have led to variable results. Moreover, in this study, I have used mice aged $\sim P 75$, whereas previous experiments by Tippmann, A. (2018) were done in mice above P100. Although in V1 of WT mice baseline spine dynamics in the apical dendrites of L5 PNs was shown to further stabilize by increasing age (Grutzendler et al., 2002), this has not been tested in PSD-95 KO mice. Overall, these results together demonstrate that PSD-95 is strongly linked to structural stability of spines.

In adult PSD-95 KO mice, elevated fraction of AMPAR-silent synapses and MD-induced spine loss in response to a brief MD constitute clear resemblances to WT CP mice. Thus, it is very likely that AMPAR-silent synapses provide the substrate for such experiencedependent spine eliminations during the $\mathrm{CP}$, although this still needs to be proven more directly. However, one of the challenges of such an experiment would be distinguishing the AMPAR-silent synapses. Currently, there are no molecular markers for AMPAR-silent synapses. Since shapes of PSD-95 KO spines are indistinguishable from their WT equivalents (Béïque et al., 2006; Migaud et al., 1998), it is also unlikely that AMPAR-silent spines have a distinct morphological identity. Thus, currently only indirect experiments can be proposed to study AMPAR-silent synapses, such as the following. Dark rearing was shown to prevent AMPAR-silent maturation (Funahashi et al., 2013) and delay the CP for 
ODP (Kang et al., 2013). Imaging in vivo spine dynamics in adult mice in which the CP for ODP is delayed into adulthood by dark rearing can provide additional insights into AMPARsilent synapse dynamics in the adult brain.

\section{4. "Plasticity brakes" and dendritic spine stability}

Several "plasticity brakes", such as chondroitin sulfate proteoglycan (CSPG), Nogo receptor 1 (NgR1), Paired immunoglobulin-like receptor B (PirB), Lynx1, class I major histocompatibility complex $(\mathrm{MHCl})$, have been suggested to limit the functional plasticity during adulthood in the context of ODP paradigm (McGee et al., 2005; Morishita et al., 2010; Pizzorusso et al., 2002; Syken et al., 2006). Tissue plasminogen activator (tPA), a major serine protease in the brain, was shown to be permissive for ODP during the critical period (Mataga et al., 2002). Among these molecules, roles of PirB, tPA and Lynx1 has been investigated in regulating the structural dynamics of spines in the context of ODP. Deletion of tPA impairs ODP and prevents MD-induced spine density reduction in apical dendrites of L2/3 PNs in juvenile mice (Mataga et al., 2004; Mataga et al., 2002). It was also shown that proteolytic activity of tPA decreases throughout the critical period for ODP and during adulthood, whereas a brief MD activates IPA only during the critical period and not beyond (Mataga et al., 2004). Later it was demonstrated that genetic removal of Lynx1 can unmask the proteolytic activity of tissue plasminogen activator (tPA) in adulthood (Bukhari et al., 2015). In Lynx1 KO adult mice (>P60), 4-d MD induced juvenile-like ODP and a nonsignificant reduction ( $\sim 25 \%$ ) in spine density at the apical dendrites of L2/3 PNs; however, the decrease in spine density was significant when the analyses were restricted to thin and stubby spines only (Bukhari et al., 2015). Both effects of 4-d MD were lost in double KO mice where tPA and Lynx1 were deleted simultaneously, suggesting that IPA is required for juvenile-like ODP in Lynx1 KO mice. In vivo imaging of spine dynamics in binocular V1 of adult Lynx1 KO mice revealed that, during baseline, both spine gain and spine elimination rates are doubled at the apical dendrites of L2/3 and L5 PNs (Sajo et al., 2016). After 4-d $M D$, in Lynx1 KO mice, there was a significant decrease in spine elimination $(95 \% \mathrm{Cl}$ : $0.019-4.262 \%, p=0.047$ ) in the apical dendrites of L5 PNs, while spine dynamics on apical dendrites of $L 2 / 3$ PNs were not affected. 4-d MD did not lead to significant changes in spine density in binocular V1 of adult Lynx1 KO mice in vivo (Sajo et al., 2016). Overall, Lynx1 KO mice show juvenile-like ODP in adulthood; however, the MD-dependent spine dynamics of adult Lynx1 KO seem to differ than that of juvenile WT mice, wherein the reduction in deprived eye responses are correlated with strong increases in spine elimination at L5 and L2/3 PNs (Sun et al., 2019; Zhou et al., 2017). 
PirB is expressed in cortical pyramidal neurons. It was identified as an MHC I molecule binding receptor in an in situ hybridization screen and was shown to limit ODP both during the critical period and adulthood (Syken et al., 2006). In mature PirB KO mice (P90), in contrast to age-matched WT controls, 3-d MD can still induce strong ODP, that is mediated by increased cortical responses to deprived-eye stimulation (Djurisic et al., 2013). Moreover, in binocular V1 of adult (P90) PirB KO mice spine density at the apical dendrites of YFP expressing L5 PNs is $\sim 43 \%$ higher than WT controls. In normally reared WT adult mice, 8-d MD induces open-eye response potentiation associated with spine formation at the apical dendrites of L5 PNs in binocular V1 (Hofer et al., 2009; Sato and Stryker, 2008; Sawtell et al., 2003). Although in adult PirB KO mice ODP could be induced faster than $8 \mathrm{~d}$ (i.e. already after 3-d MD), the spine density in the apical dendrites of L5 PN remained stable during this period (Djurisic et al., 2013). The authors concluded that in adult PirB KO mice new spine formation might be occluded by already existing high density of spines in the apical dendrites of L5 PNs. More recently, it was shown that spine density is also elevated in apical and basal dendrites of L2/3 PNs in the binocular V1 of P30 PirB KO mice, and that PirB expression regulates the spine density cell-autonomously. However, the effects of MD on spine density in L2/3 PNs was not addressed in this study (Vidal et al., 2016).

In summary, although removal of abovementioned plasticity breaks enhances ODP in adulthood, MD-dependent changes in structural dynamics of spines do not resemble that of juvenile mice described recently (Sun et al., 2019; Zhou et al., 2017). On the other hand, in adult PSD-95-deficient mice, MD led to a rapid spine elimination in apical dendrites of L2/3 PN in binocular V1, similar to what has been reported in juvenile mice (Sun et al., 2019). Thus, it seems that removal of plasticity brakes is permissive for expression of adultlike ODP, whereas deletion of PSD-95 in adult mice confers circuits in binocular V1 a juvenile-like plasticity. It would be interesting to investigate the role of PSD-95 in ODP in relation to abovementioned plasticity breaks. One could hypothesize that removal of plasticity breaks and PSD-95 simultaneously, could have a cumulative effect on enhancing ODP and the underlying structural dynamics in adult mice.

\subsection{Role of neurogranin in the critical period closure for ODP and visual acuity}

Previously it was shown that preventing maturation of AMPAR-silent synapses in V1 by deleting PSD-95 impairs the closure of CP for ODP, and in PSD-95 KO mice juvenile-like ODP is preserved lifelong (Huang et al., 2015). More recently, it was demonstrated that neurogranin is also necessary for experience-dependent AMPAR-silent synapse maturation 
in the binocular V1 of mice (Han et al,. 2017). In addition, neurogranin was shown to keep AMPAR-mediated signaling in equilibrium during the CP through coordinating experiencedependent spine pruning (Han et al., 2017). Similar to PSD-95-deficient mice, loss of neurogranin leads to elevated fraction of AMPAR-silent synapses (Han et al., 2017). Inspired by these results, I have set out to investigate whether neurogranin is also required in V1 for closure of the CP for ODP. Here I show that, indeed, silencing expression of neurogranin in V1 postnatally via a virus mediated knockdown impaired the CP closure for ODP and preserved juvenile-like ODP into adulthood (P49-P83). In young adult neurogranin-knockdown mice ( P67), a brief (4-d) MD was sufficient to induce strong OD shifts. Although in neurogranin knockdown mice without MD binocular V1 was dominated by contralateral eye input, after 4-d MD, cortical responses to contralateral eye stimulation were lower and no longer significantly different from V1 responses to ipsilateral eye stimulation. Next, I tested whether silencing neurogranin expression would enhance ODP even in an older age. In normally reared mice, ODP can be induced after the closure of CP if the duration of $M D$ is extended to $7-d M D$, whereas beyond P110, 7-d is no longer sufficient to induce ODP (Lehmann and Löwel, 2008). I found that in fully adult control knockdown mice above P110 ( P135), V1 was still dominated by the contralateral eye input after 7-d MD, as expected. In neurogranin knockdown mice, however, this was not the case, and OD values were significantly lower (shifted towards 0 ) than control mice with 7-d MD. Binocular V1 responses to deprived eye stimulation were significantly lower in adult neurogranin-knockdown mice than that of control mice, while open eye responses were similar. Thus, silencing neurogranin expression enhanced ODP in fully adult mice well beyond the CP for ODP. Moreover, topographic mapping of $\mathrm{V} 1$ in neurogranin and control knockdown mice were normal. Interestingly, maximum $\mathrm{V} 1$ activities in response to elevation stimulation of the contralateral eye were significantly reduced in neurogranin knockdown mice, supporting previous observations (Han et al., 2017; Pak et al., 2000). Overall, these results provide additional evidence for the regulation of the CP for ODP by a mechanism based on AMPAR-silent synapse maturation.

It still needs to be tested whether in fully mature neurogranin knockdown mice a 4-d MD would also be sufficient to induce juvenile-like ODP, as seen in PSD-95 deficient mice (Huang et al., 2015). Moreover, Han et al. (2017) investigated the AMPAR-silent synapses only until P30, and it needs to be confirmed whether the AMPAR-silent synapses remain elevated in neurogranin-deficient mice in adulthood. Given the juvenile-like ODP of adult neurogranin-deficient mice and the previous results from PSD-95-deficient mice (Huang et al., 2015), this is highly likely. Finally, it needs to be examined whether acute knockdown of 
neurogranin in adult mice can reinstate the juvenile plasticity after the CP for ODP has already been closed.

I have also tested the visual capabilities of neurogranin-knockdown mice in two behavioral tests, namely the visual water task (VWT) and the optomotor setup (Prusky et al., 2006; Prusky et al., 2000). Testing in the VWT revealed that the visual acuity of neurograninknockdown mice ( P70) was at 0.46 cyc/deg, significantly lower than that of age matched control knockdown mice (0.67 cyc/deg). The visual acuity of the control group was comparable with a recent study, which reported an average visual acuity of 0.64 cyc/deg in P100 mice (Hosang et al., 2018). Impaired visual acuity of neurogranin-knockdown mice is also in line with the impaired visually guided behavior of these mice in the visual cliff test (Han et al., 2017). On the other hand, in comparison to control group, neurograninknockdown mice showed nearly identical optomotor reflex thresholds for spatial frequency and contrast sensitivity in the optomotor test before and after MD. Optomotor responses are mediated by subcortical pathways (Giolli et al., 2006); however, the MD-induced increases in the optomotor reflexes are dependent on cortical projections of $\mathrm{V} 1$ to brainstem accessory optic system (Liu et al., 2016; Prusky et al., 2004). Outcomes of these two behavioral tests show that neurogranin is important in V1 for achieving the optimal visual acuity, and the function of cortical projections in mediating MD-dependent reflex enhancements are not affected by the loss of neurogranin.

\subsection{PSD-95 and neurogranin}

Results of this thesis in conjunction with previous reports (Han et al., 2017; Huang et al., 2015) show that both PSD-95 and neurogranin regulate CP closure by promoting the maturation of AMPAR-silent synapses. In order to understand the mechanistic roles of PSD95 and neurogranin in cortical maturation more thoroughly, two results that pointed out potential differences between neurogranin and PSD-95 functions need to be addressed: 1) maximum V1 activation after elevation stimulation of the contralateral eye in neurograninknockdown mice was lower than controls, but the quality of elevation maps did not differ; 2) V1-targeted knockdown of neurogranin led to a visual acuity impairment in the visual water task (VWT), while the visual acuity of PSD-95. KO mice does not differ from WT controls (Huang et al., 2015).

Although loss of PSD-95 and neurogranin both impair maturation of AMPAR-silent synapses, in neurogranin knockdown mice, V1 activation of the elevation maps was lower than controls. V1 activity in elevation maps of PSD-95 KO was, on the contrary, higher than 
WT controls (Huang et al., 2015). Reduced V1 activity in neurogranin-knockdown mice also corroborates the previous findings where magnitude of field excitatory postsynaptic potentials in L4 - L2/3 circuitry of V1 was $\sim 50 \%$ lower than WT mice (Han et al., 2017). Another considerable difference between neurogranin and PSD-95 deficient mice is the spine density of L2/3 PNs (Han et al., 2017). In the basal dendrites of L2/3 PNs, Han et al. (2017) observed increased spine loss during the development (Han et al., 2017), which could explain the lower $\mathrm{V} 1$ activation in adult mice in elevation maps, as well as the visual acuity impairment. Testing in the VWT was shown to increase spine density in basal and apical dendrites of L2/3 PNs in V1 of adult mice (Wang et al., 2016). Thus, spine loss in neurogranin knockdown mice may have prevented these mice reaching the visual acuity values seen in the control group. Moreover, impairing the pace of AMPAR-silent synapse maturation via genetic modifications or dark-rearing does not affect visual acuity (Favaro et al., 2018; Kang et al., 2013; Rochefort et al., 2011). Thus, in neurogranin knockdown mice, impaired visual acuity is most likely not due to elevated fraction of AMPAR-silent synapses, but rather due to an exacerbated loss of spines as reported before (Han et al., 2017). In conclusion, neurogranin and PSD-95 are both required for the CP closure, but we also see that PSD-95 and neurogranin have additional non-overlapping functions. This is not surprising due to their different roles in the post-synaptic machinery. Nevertheless, by studying the role of neurogranin in $\mathrm{CP}$ closure for ODP, findings in this thesis further consolidate the idea that CP closure for ODP is mediated by maturation of AMPAR-silent synapses. 


\section{References}

Abbas, A.I., Yadav, P.N., Yao, W.D., Arbuckle, M.I., Grant, S.G., Caron, M.G., and Roth, B.L. (2009). PSD-95 is essential for hallucinogen and atypical antipsychotic drug actions at serotonin receptors. J Neurosci 29, 7124-7136.

Angevine, J.B., Jr., and Sidman, R.L. (1961). Autoradiographic study of cell migration during histogenesis of cerebral cortex in the mouse. Nature 192, 766-768.

Antonini, A., Fagiolini, M., and Stryker, M.P. (1999). Anatomical correlates of functional plasticity in mouse visual cortex. J Neurosci 19, 4388-4406.

Antonini, A., and Stryker, M.P. (1993). Rapid remodeling of axonal arbors in the visual cortex. Science 260, 1819-1821.

Baudier, J., Bronner, C., Kligman, D., and Cole, R.D. (1989). Protein kinase C substrates from bovine brain. Purification and characterization of neuromodulin, a neuronspecific calmodulin-binding protein. J Biol Chem 264, 1824-1828.

Béïque, J.C., and Andrade, R. (2003). PSD-95 regulates synaptic transmission and plasticity in rat cerebral cortex. J Physiol 546, 859-867.

Béïque, J.C., Lin, D.T., Kang, M.G., Aizawa, H., Takamiya, K., and Huganir, R.L. (2006). Synapse-specific regulation of AMPA receptor function by PSD-95. Proc Natl Acad Sci U S A 103, 19535-19540.

Berry, K.P., and Nedivi, E. (2016). Experience-Dependent Structural Plasticity in the Visual System. Annu Rev Vis Sci 2, 17-35.

Berry, K.P., and Nedivi, E. (2017). Spine Dynamics: Are They All the Same? Neuron 96, 43-55.

Birch, E.E. (2013). Amblyopia and binocular vision. Prog Retin Eye Res 33, 67-84.

Blakemore, C., Garey, L.J., and Vital-Durand, F. (1978). The physiological effects of monocular deprivation and their reversal in the monkey's visual cortex. J Physiol 283, 223-262.

Böhner, J. (1990). Early acquisition of song in the zebra finch, Taeniopygia guttata. Animal Behaviour 39, 369-374.

Bourgeron, T. (2015). From the genetic architecture to synaptic plasticity in autism spectrum disorder. Nat Rev Neurosci 16, 551-563.

Brainard, M.S., and Doupe, A.J. (2002). What songbirds teach us about learning. Nature 417, 351-358.

Briner, A., De Roo, M., Dayer, A., Muller, D., Habre, W., and Vutskits, L. (2010). Volatile anesthetics rapidly increase dendritic spine density in the rat medial prefrontal cortex during synaptogenesis. Anesthesiology 112, 546-556. 
Bukhari, N., Burman, P.N., Hussein, A., Demars, M.P., Sadahiro, M., Brady, D.M., Tsirka, S.E., Russo, S.J., and Morishita, H. (2015). Unmasking Proteolytic Activity for Adult Visual Cortex Plasticity by the Removal of Lynx1. J Neurosci 35, 12693-12702.

Busse, L. (2018). Chapter 4 - The Mouse Visual System and Visual Perception. In Handbook of Behavioral Neuroscience, A. Ennaceur, and M.A. de Souza Silva, eds. (Elsevier), pp. 53-68.

Cajal, S.R.y. (1888). Estructura de los centros nerviosos de las aves. . Rev Trim Histol Norm 1, 1-10.

Cajal, S.R.y. (1894). The Croonian Lecture: La Fine Structure des Centres Nerveux. Proceedings of the Royal Society of London 55, 444-468.

Callaway, E.M. (1998). Local circuits in primary visual cortex of the macaque monkey. Annu Rev Neurosci 21, 47-74.

Cane, M., Maco, B., Knott, G., and Holtmaat, A. (2014). The relationship between PSD-95 clustering and spine stability in vivo. J Neurosci 34, 2075-2086.

Cang, J., Kalatsky, V.A., Löwel, S., and Stryker, M.P. (2005a). Optical imaging of the intrinsic signal as a measure of cortical plasticity in the mouse. Vis Neurosci 22, 685691.

Cang, J., Rentería, R.C., Kaneko, M., Liu, X., Copenhagen, D.R., and Stryker, M.P. (2005b). Development of precise maps in visual cortex requires patterned spontaneous activity in the retina. Neuron 48, 797-809.

Carlisle, H.J., Fink, A.E., Grant, S.G., and O'Dell, T.J. (2008). Opposing effects of PSD-93 and PSD-95 on long-term potentiation and spike timing-dependent plasticity. J Physiol 586, 5885-5900.

Carmignoto, G., and Vicini, S. (1992). Activity-dependent decrease in NMDA receptor responses during development of the visual cortex. Science 258, 1007-1011.

Chalfie, M., Tu, Y., Euskirchen, G., Ward, W.W., and Prasher, D.C. (1994). Green fluorescent protein as a marker for gene expression. Science 263, 802-805.

Chen, J.L., Villa, K.L., Cha, J.W., So, P.T., Kubota, Y., and Nedivi, E. (2012). Clustered dynamics of inhibitory synapses and dendritic spines in the adult neocortex. Neuron 74, 361-373.

Chen, L., Chetkovich, D.M., Petralia, R.S., Sweeney, N.T., Kawasaki, Y., Wenthold, R.J., Bredt, D.S., and Nicoll, R.A. (2000). Stargazin regulates synaptic targeting of AMPA receptors by two distinct mechanisms. Nature 408, 936-943.

Cho, K.O., Hunt, C.A., and Kennedy, M.B. (1992). The rat brain postsynaptic density fraction contains a homolog of the Drosophila discs-large tumor suppressor protein. Neuron 9, 929-942. 
Dailey, M.E., and Smith, S.J. (1996). The dynamics of dendritic structure in developing hippocampal slices. J Neurosci 16, 2983-2994.

De Paola, V., Holtmaat, A., Knott, G., Song, S., Wilbrecht, L., Caroni, P., and Svoboda, K. (2006). Cell type-specific structural plasticity of axonal branches and boutons in the adult neocortex. Neuron 49, 861-875.

De Roo, M., Klauser, P., Briner, A., Nikonenko, I., Mendez, P., Dayer, A., Kiss, J.Z., Muller, D., and Vutskits, L. (2009). Anesthetics rapidly promote synaptogenesis during a critical period of brain development. PLoS One 4, e7043.

De Roo, M., Klauser, P., Mendez, P., Poglia, L., and Muller, D. (2008). Activity-dependent PSD formation and stabilization of newly formed spines in hippocampal slice cultures. Cereb Cortex 18, 151-161.

DeFelipe, J., Conti, F., Van Eyck, S.L., and Manzoni, T. (1988). Demonstration of glutamate-positive axon terminals forming asymmetric synapses in cat neocortex. Brain Res 455, 162-165.

Denk, W., Strickler, J.H., and Webb, W.W. (1990). Two-photon laser scanning fluorescence microscopy. Science 248, 73-76.

Denk, W., and Svoboda, K. (1997). Photon upmanship: why multiphoton imaging is more than a gimmick. Neuron 18, 351-357.

Djurisic, M., Vidal, G.S., Mann, M., Aharon, A., Kim, T., Ferrao Santos, A., Zuo, Y., Hubener, M., and Shatz, C.J. (2013). PirB regulates a structural substrate for cortical plasticity. Proc Natl Acad Sci U S A 110, 20771-20776.

Dräger, U.C. (1975). Receptive fields of single cells and topography in mouse visual cortex. J Comp Neurol 160, 269-290.

Dräger, U.C. (1978). Observations on monocular deprivation in mice. J Neurophysiol 41, 28-42.

Dräger, U.C., and Olsen, J.F. (1980). Origins of crossed and uncrossed retinal projections in pigmented and albino mice. J Comp Neurol 191, 383-412.

Ehrlich, I., Klein, M., Rumpel, S., and Malinow, R. (2007). PSD-95 is required for activitydriven synapse stabilization. Proc Natl Acad Sci U S A 104, 4176-4181.

El-Husseini, A.E., Schnell, E., Chetkovich, D.M., Nicoll, R.A., and Bredt, D.S. (2000). PSD95 involvement in maturation of excitatory synapses. Science 290, 1364-1368.

Elias, G.M., Funke, L., Stein, V., Grant, S.G., Bredt, D.S., and Nicoll, R.A. (2006). Synapsespecific and developmentally regulated targeting of AMPA receptors by a family of MAGUK scaffolding proteins. Neuron 52, 307-320.

Espinosa, J.S., and Stryker, M.P. (2012). Development and plasticity of the primary visual cortex. Neuron 75, 230-249. 
Fagiolini, M., Fritschy, J.M., Low, K., Mohler, H., Rudolph, U., and Hensch, T.K. (2004). Specific GABAA circuits for visual cortical plasticity. Science 303, 1681-1683.

Fagiolini, M., and Hensch, T.K. (2000). Inhibitory threshold for critical-period activation in primary visual cortex. Nature 404, 183-186.

Favaro, P.D., Huang, X., Hosang, L., Stodieck, S., Cui, L., Liu, Y.Z., Engelhardt, K.A., Schmitz, F., Dong, Y., Löwel, S., et al. (2018). An opposing function of paralogs in balancing developmental synapse maturation. PLoS Biol 16, e2006838.

Feldman, D.E., Brainard, M.S., and Knudsen, E.I. (1996). Newly learned auditory responses mediated by NMDA receptors in the owl inferior colliculus. Science 271, 525-528.

Feldmeyer, D., Lübke, J., Silver, R.A., and Sakmann, B. (2002). Synaptic connections between layer 4 spiny neurone-layer $2 / 3$ pyramidal cell pairs in juvenile rat barrel cortex: physiology and anatomy of interlaminar signalling within a cortical column. $J$ Physiol 538, 803-822.

Feng, G., Mellor, R.H., Bernstein, M., Keller-Peck, C., Nguyen, Q.T., Wallace, M., Nerbonne, J.M., Lichtman, J.W., and Sanes, J.R. (2000). Imaging neuronal subsets in transgenic mice expressing multiple spectral variants of GFP. Neuron 28, 41-51.

Flege, J.E., Munro, M.J., and MacKay, I.R. (1995). Factors affecting strength of perceived foreign accent in a second language. J Acoust Soc Am 97, 3125-3134.

Frenkel, M.Y., and Bear, M.F. (2004). How Monocular Deprivation Shifts Ocular Dominance in Visual Cortex of Young Mice. Neuron 44, 917-923.

Frenkel, M.Y., Sawtell, N.B., Diogo, A.C., Yoon, B., Neve, R.L., and Bear, M.F. (2006). Instructive effect of visual experience in mouse visual cortex. Neuron 51, 339-349.

Fu, M., and Zuo, Y. (2011). Experience-dependent structural plasticity in the cortex. Trends Neurosci 34, 177-187.

Fukata, Y., Tzingounis, A.V., Trinidad, J.C., Fukata, M., Burlingame, A.L., Nicoll, R.A., and Bredt, D.S. (2005). Molecular constituents of neuronal AMPA receptors. J Cell Biol 169, 399-404.

Funahashi, R., Maruyama, T., Yoshimura, Y., and Komatsu, Y. (2013). Silent synapses persist into adulthood in layer 2/3 pyramidal neurons of visual cortex in dark-reared mice. J Neurophysiol 109, 2064-2076.

Gaertner, T.R., Putkey, J.A., and Waxham, M.N. (2004). RC3/Neurogranin and $\mathrm{Ca} 2+/$ calmodulin-dependent protein kinase II produce opposing effects on the affinity of calmodulin for calcium. J Biol Chem 279, 39374-39382.

Garcia-Lopez, P., Garcia-Marin, V., and Freire, M. (2007). The discovery of dendritic spines by Cajal in 1888 and its relevance in the present neuroscience. Prog Neurobiol 83, 110-130. 
Gianfranceschi, L., Siciliano, R., Walls, J., Morales, B., Kirkwood, A., Huang, Z.J., Tonegawa, S., and Maffei, L. (2003). Visual cortex is rescued from the effects of dark rearing by overexpression of BDNF. Proc Natl Acad Sci U S A 100, 1248612491.

Gilbert, C.D. (1983). Microcircuitry of the visual cortex. Annu Rev Neurosci 6, 217-247.

Giolli, R.A., Blanks, R.H., and Lui, F. (2006). The accessory optic system: basic organization with an update on connectivity, neurochemistry, and function. Prog Brain Res 151, 407-440.

Gordon, J.A., and Stryker, M.P. (1996). Experience-dependent plasticity of binocular responses in the primary visual cortex of the mouse. J Neurosci 16, 3274-3286.

Gray, E.G. (1959). Electron microscopy of synaptic contacts on dendrite spines of the cerebral cortex. Nature 183, 1592-1593.

Gray, N.W., Weimer, R.M., Bureau, I., and Svoboda, K. (2006). Rapid redistribution of synaptic PSD-95 in the neocortex in vivo. PLoS Biol 4, e370.

Greenberg, D.S., and Kerr, J.N. (2009). Automated correction of fast motion artifacts for two-photon imaging of awake animals. J Neurosci Methods 176, 1-15.

Greifzu, F., Kalogeraki, E., and Löwel, S. (2016). Environmental enrichment preserved lifelong ocular dominance plasticity, but did not improve visual abilities. Neurobiol Aging 41, 130-137.

Greifzu, F., Pielecka-Fortuna, J., Kalogeraki, E., Krempler, K., Favaro, P.D., Schlüter, O.M., and Löwel, S. (2014). Environmental enrichment extends ocular dominance plasticity into adulthood and protects from stroke-induced impairments of plasticity. Proc Natl Acad Sci U S A 111, 1150-1155.

Greifzu, F., Wolf, F., and Löwel, S. (2012). Network influences on cortical plasticity. Neuroforum 18, 41-48.

Grutzendler, J., Kasthuri, N., and Gan, W.B. (2002). Long-term dendritic spine stability in the adult cortex. Nature 420, 812-816.

Hall, J., Trent, S., Thomas, K.L., O'Donovan, M.C., and Owen, M.J. (2015). Genetic risk for schizophrenia: convergence on synaptic pathways involved in plasticity. Biol Psychiatry 77, 52-58.

Han, K.S., Cooke, S.F., and Xu, W. (2017). Experience-Dependent Equilibration of AMPARMediated Synaptic Transmission during the Critical Period. Cell Rep 18, 892-904.

Harauzov, A., Spolidoro, M., DiCristo, G., De Pasquale, R., Cancedda, L., Pizzorusso, T., Viegi, A., Berardi, N., and Maffei, L. (2010). Reducing intracortical inhibition in the adult visual cortex promotes ocular dominance plasticity. J Neurosci 30, 361-371.

Harris, K.M., Jensen, F.E., and Tsao, B. (1992). Three-dimensional structure of dendritic spines and synapses in rat hippocampus (CA1) at postnatal day 15 and adult ages: 
implications for the maturation of synaptic physiology and long-term potentiation. $J$ Neurosci 12, 2685-2705.

Hefendehl, J.K., Milford, D., Eicke, D., Wegenast-Braun, B.M., Calhoun, M.E., Grathwohl, S.A., Jucker, M., and Liebig, C. (2012). Repeatable target localization for long-term in vivo imaging of mice with 2-photon microscopy. J Neurosci Methods 205, 357363.

Hensch, T.K. (2005). Critical period plasticity in local cortical circuits. Nat Rev Neurosci 6, 877-888.

Hensch, T.K., Fagiolini, M., Mataga, N., Stryker, M.P., Baekkeskov, S., and Kash, S.F. (1998). Local GABA circuit control of experience-dependent plasticity in developing visual cortex. Science 282, 1504-1508.

Hensch, T.K., and Quinlan, E.M. (2018). Critical periods in amblyopia. Vis Neurosci 35, E014.

Hofer, S.B., Mrsic-Flogel, T.D., Bonhoeffer, T., and Hübener, M. (2006). Prior experience enhances plasticity in adult visual cortex. Nat Neurosci 9, 127-132.

Hofer, S.B., Mrsic-Flogel, T.D., Bonhoeffer, T., and Hübener, M. (2009). Experience leaves a lasting structural trace in cortical circuits. Nature 457, 313-317.

Holtmaat, A., Bonhoeffer, T., Chow, D.K., Chuckowree, J., De Paola, V., Hofer, S.B., Hübener, M., Keck, T., Knott, G., Lee, W.C., et al. (2009). Long-term, high-resolution imaging in the mouse neocortex through a chronic cranial window. Nat Protoc 4, 1128-1144.

Holtmaat, A., and Svoboda, K. (2009). Experience-dependent structural synaptic plasticity in the mammalian brain. Nat Rev Neurosci 10, 647-658.

Holtmaat, A., Wilbrecht, L., Knott, G.W., Welker, E., and Svoboda, K. (2006). Experiencedependent and cell-type-specific spine growth in the neocortex. Nature 441, 979983.

Holtmaat, A.J., Trachtenberg, J.T., Wilbrecht, L., Shepherd, G.M., Zhang, X., Knott, G.W., and Svoboda, K. (2005). Transient and persistent dendritic spines in the neocortex in vivo. Neuron 45, 279-291.

Hosang, L., Yusifov, R., and Löwel, S. (2018). Long-Term Visual Training Increases Visual Acuity and Long-Term Monocular Deprivation Promotes Ocular Dominance Plasticity in Adult Standard Cage-Raised Mice. eNeuro 5.

Huang, X., Stodieck, S.K., Goetze, B., Cui, L., Wong, M.H., Wenzel, C., Hosang, L., Dong, Y., Löwel, S., and Schlüter, O.M. (2015). Progressive maturation of silent synapses governs the duration of a critical period. Proc Natl Acad Sci U S A 112, E3131-3140. 
Huang, Z.J., Kirkwood, A., Pizzorusso, T., Porciatti, V., Morales, B., Bear, M.F., Maffei, L., and Tonegawa, S. (1999). BDNF regulates the maturation of inhibition and the critical period of plasticity in mouse visual cortex. Cell 98, 739-755.

Hubel, D.H., and Wiesel, T.N. (1962). Receptive fields, binocular interaction and functional architecture in the cat's visual cortex. J Physiol 160, 106-154.

Hubel, D.H., and Wiesel, T.N. (1963). Receptive fields of cells in striate cortex of very young, visually inexperienced kittens. J Neurophysiol 26, 994-1002.

Hubel, D.H., and Wiesel, T.N. (1970). The period of susceptibility to the physiological effects of unilateral eye closure in kittens. J Physiol 206, 419-436.

Hubel, D.H., Wiesel, T.N., and LeVay, S. (1976). Functional architecture of area 17 in normal and monocularly deprived macaque monkeys. Cold Spring Harb Symp Quant Biol 40, 581-589.

Hubel, D.H., Wiesel, T.N., and LeVay, S. (1977). Plasticity of ocular dominance columns in monkey striate cortex. Philos Trans R Soc Lond B Biol Sci 278, 377-409.

Hwang, H., Szucs, M.J., Ding, L.J., Allen, A., Haensgen, H., Gao, F., Andrade, A., Pan, J.Q., Carr, S.A., Ahmad, R., et al. (2018). A schizophrenia risk gene, NRGN, bidirectionally modulates synaptic plasticity via regulating the neuronal phosphoproteome. bioRxiv.

Isaac, J.T., Nicoll, R.A., and Malenka, R.C. (1995). Evidence for silent synapses: implications for the expression of LTP. Neuron 15, 427-434.

Issa, N.P., Trachtenberg, J.T., Chapman, B., Zahs, K.R., and Stryker, M.P. (1999). The critical period for ocular dominance plasticity in the Ferret's visual cortex. J Neurosci 19, 6965-6978.

Itami, C., Kimura, F., Kohno, T., Matsuoka, M., Ichikawa, M., Tsumoto, T., and Nakamura, S. (2003). Brain-derived neurotrophic factor-dependent unmasking of "silent" synapses in the developing mouse barrel cortex. Proc Natl Acad Sci U S A 100, 13069-13074.

Jeromin, A., Yuan, L.L., Frick, A., Pfaffinger, P., and Johnston, D. (2003). A modified Sindbis vector for prolonged gene expression in neurons. J Neurophysiol 90, 2741-2745.

Joachimsthaler, B., Brugger, D., Skodras, A., and Schwarz, C. (2015). Spine loss in primary somatosensory cortex during trace eyeblink conditioning. J Neurosci 35, 3772-3781.

Johnson, J.S., and Newport, E.L. (1989). Critical period effects in second language learning: The influence of maturational state on the acquisition of English as a second language. Cognitive Psychology 21, 60-99.

Kaech, S., Brinkhaus, H., and Matus, A. (1999). Volatile anesthetics block actin-based motility in dendritic spines. Proc Natl Acad Sci U S A 96, 10433-10437. 
Kalatsky, V.A., and Stryker, M.P. (2003). New paradigm for optical imaging: temporally encoded maps of intrinsic signal. Neuron 38, 529-545.

Kalogeraki, E., Yusifov, R., and Löwel, S. (2019). Transgenerational Transmission of Enhanced Ocular Dominance Plasticity from Enriched Mice to Their Non-enriched Offspring. eNeuro 6.

Kaneko, M., Hanover, J.L., England, P.M., and Stryker, M.P. (2008). TrkB kinase is required for recovery, but not loss, of cortical responses following monocular deprivation. Nat Neurosci 11, 497-504.

Kang, E., Durand, S., LeBlanc, J.J., Hensch, T.K., Chen, C., and Fagiolini, M. (2013). Visual acuity development and plasticity in the absence of sensory experience. J Neurosci 33, 17789-17796.

Kanold, P.O., Kim, Y.A., GrandPre, T., and Shatz, C.J. (2009). Co-regulation of ocular dominance plasticity and NMDA receptor subunit expression in glutamic acid decarboxylase-65 knock-out mice. J Physiol 587, 2857-2867.

Katz, L.C., and Shatz, C.J. (1996). Synaptic activity and the construction of cortical circuits. Science 274, 1133-1138.

Keck, T., Mrsic-Flogel, T.D., Vaz Afonso, M., Eysel, U.T., Bonhoeffer, T., and Hubener, M. (2008). Massive restructuring of neuronal circuits during functional reorganization of adult visual cortex. Nat Neurosci 11, 1162-1167.

Klausberger, T., Roberts, J.D., and Somogyi, P. (2002). Cell type- and input-specific differences in the number and subtypes of synaptic $\operatorname{GABA}(A)$ receptors in the hippocampus. J Neurosci 22, 2513-2521.

Knudsen, E.I., Knudsen, P.F., and Esterly, S.D. (1982). Early auditory experience modifies sound localization in barn owls. Nature 295, 238-240.

Knudsen, E.I., and Konishi, M. (1979). Mechanisms of sound localization in the barn owl (Tyto alba). Journal of comparative physiology 133, 13-21.

Kornau, H.C., Schenker, L.T., Kennedy, M.B., and Seeburg, P.H. (1995). Domain interaction between NMDA receptor subunits and the postsynaptic density protein PSD-95. Science 269, 1737-1740.

Lehmann, K., and Löwel, S. (2008). Age-dependent ocular dominance plasticity in adult mice. PLoS One 3, e3120.

Lendvai, B., Stern, E.A., Chen, B., and Svoboda, K. (2000). Experience-dependent plasticity of dendritic spines in the developing rat barrel cortex in vivo. Nature 404, 876-881.

LeVay, S. (1973). Synaptic patterns in the visual cortex of the cat and monkey. Electron microscopy of Golgi preparations. J Comp Neurol 150, 53-85. 
Levelt, C.N., and Hübener, M. (2012). Critical-period plasticity in the visual cortex. Annu Rev Neurosci 35, 309-330.

Liao, D., Hessler, N.A., and Malinow, R. (1995). Activation of postsynaptically silent synapses during pairing-induced LTP in CA1 region of hippocampal slice. Nature 375, 400-404.

Liu, B.H., Huberman, A.D., and Scanziani, M. (2016). Cortico-fugal output from visual cortex promotes plasticity of innate motor behaviour. Nature 538, 383-387.

Lorenz, K.Z. (1958). The evolution of behavior. Sci Am 199, 67-74 passim.

Löwel, S., and Singer, W. (1992). Selection of intrinsic horizontal connections in the visual cortex by correlated neuronal activity. Science 255, 209-212.

Ma, L., Qiao, Q., Tsai, J.W., Yang, G., Li, W., and Gan, W.B. (2016). Experience-dependent plasticity of dendritic spines of layer $2 / 3$ pyramidal neurons in the mouse cortex. Dev Neurobiol 76, 277-286.

Majewska, A.K., Newton, J.R., and Sur, M. (2006). Remodeling of synaptic structure in sensory cortical areas in vivo. J Neurosci 26, 3021-3029.

Malinow, R., Madison, D.V., and Tsien, R.W. (1988). Persistent protein kinase activity underlying long-term potentiation. Nature 335, 820-824.

Mataga, N., Mizuguchi, Y., and Hensch, T.K. (2004). Experience-dependent pruning of dendritic spines in visual cortex by tissue plasminogen activator. Neuron 44, 1031 1041.

Mataga, N., Nagai, N., and Hensch, T.K. (2002). Permissive proteolytic activity for visual cortical plasticity. Proc Natl Acad Sci U S A 99, 7717-7721.

McGee, A.W., Yang, Y., Fischer, Q.S., Daw, N.W., and Strittmatter, S.M. (2005). Experience-driven plasticity of visual cortex limited by myelin and Nogo receptor. Science 309, 2222-2226.

Meyer, T., Hanson, P.I., Stryer, L., and Schulman, H. (1992). Calmodulin trapping by calcium-calmodulin-dependent protein kinase. Science 256, 1199-1202.

Migaud, M., Charlesworth, P., Dempster, M., Webster, L.C., Watabe, A.M., Makhinson, M., He, Y., Ramsay, M.F., Morris, R.G., Morrison, J.H., et al. (1998). Enhanced longterm potentiation and impaired learning in mice with mutant postsynaptic density-95 protein. Nature 396, 433-439.

Mioche, L., and Singer, W. (1989). Chronic recordings from single sites of kitten striate cortex during experience-dependent modifications of receptive-field properties. $J$ Neurophysiol 62, 185-197.

Miquelajauregui, A., Kribakaran, S., Mostany, R., Badaloni, A., Consalez, G.G., and Portera-Cailliau, C. (2015). Layer 4 pyramidal neurons exhibit robust dendritic spine plasticity in vivo after input deprivation. J Neurosci 35, 7287-7294. 
Morishita, H., Miwa, J.M., Heintz, N., and Hensch, T.K. (2010). Lynx1, a cholinergic brake, limits plasticity in adult visual cortex. Science 330, 1238-1240.

Moyer, C.E., and Zuo, Y. (2018). Cortical dendritic spine development and plasticity: insights from in vivo imaging. Curr Opin Neurobiol 53, 76-82.

Mrsic-Flogel, T.D., Hofer, S.B., Ohki, K., Reid, R.C., Bonhoeffer, T., and Hubener, M. (2007). Homeostatic regulation of eye-specific responses in visual cortex during ocular dominance plasticity. Neuron 54, 961-972.

Mulkey, R.M., Endo, S., Shenolikar, S., and Malenka, R.C. (1994). Involvement of a calcineurin/inhibitor-1 phosphatase cascade in hippocampal long-term depression. Nature 369, 486-488.

Nakazawa, T., Komai, S., Tezuka, T., Hisatsune, C., Umemori, H., Semba, K., Mishina, M., Manabe, T., and Yamamoto, T. (2001). Characterization of Fyn-mediated tyrosine phosphorylation sites on GluR epsilon 2 (NR2B) subunit of the N-methyl-D-aspartate receptor. J Biol Chem 276, 693-699.

Oray, S., Majewska, A., and Sur, M. (2004). Dendritic spine dynamics are regulated by monocular deprivation and extracellular matrix degradation. Neuron 44, 1021-1030.

Pak, J.H., Huang, F.L., Li, J., Balschun, D., Reymann, K.G., Chiang, C., Westphal, H., and Huang, K.P. (2000). Involvement of neurogranin in the modulation of calcium/calmodulin-dependent protein kinase II, synaptic plasticity, and spatial learning: a study with knockout mice. Proc Natl Acad Sci U S A 97, 11232-11237.

Peebles, C.L., Yoo, J., Thwin, M.T., Palop, J.J., Noebels, J.L., and Finkbeiner, S. (2010). Arc regulates spine morphology and maintains network stability in vivo. Proc Natl Acad Sci U S A 107, 18173-18178.

Petersen, A., and Gerges, N.Z. (2015). Neurogranin regulates CaM dynamics at dendritic spines. Sci Rep 5, 11135.

Pizzorusso, T., Medini, P., Berardi, N., Chierzi, S., Fawcett, J.W., and Maffei, L. (2002). Reactivation of ocular dominance plasticity in the adult visual cortex. Science 298, 1248-1251.

Platholi, J., Herold, K.F., Hemmings, H.C., Jr., and Halpain, S. (2014). Isoflurane reversibly destabilizes hippocampal dendritic spines by an actin-dependent mechanism. PLoS One 9, e102978.

Pologruto, T.A., Sabatini, B.L., and Svoboda, K. (2003). Scanlmage: flexible software for operating laser scanning microscopes. Biomed Eng Online 2, 13.

Prusky, G.T., Alam, N.M., Beekman, S., and Douglas, R.M. (2004). Rapid quantification of adult and developing mouse spatial vision using a virtual optomotor system. Invest Ophthalmol Vis Sci 45, 4611-4616. 
Prusky, G.T., Alam, N.M., and Douglas, R.M. (2006). Enhancement of vision by monocular deprivation in adult mice. J Neurosci 26, 11554-11561.

Prusky, G.T., West, P.W., and Douglas, R.M. (2000). Behavioral assessment of visual acuity in mice and rats. Vision Res 40, 2201-2209.

Quinlan, E.M., Olstein, D.H., and Bear, M.F. (1999). Bidirectional, experience-dependent regulation of $\mathrm{N}$-methyl-D-aspartate receptor subunit composition in the rat visual cortex during postnatal development. Proc Natl Acad Sci U S A 96, 12876-12880.

Represa, A., Deloulme, J.C., Sensenbrenner, M., Ben-Ari, Y., and Baudier, J. (1990). Neurogranin: immunocytochemical localization of a brain-specific protein kinase $\mathrm{C}$ substrate. J Neurosci 10, 3782-3792.

Riedl, J., Crevenna, A.H., Kessenbrock, K., Yu, J.H., Neukirchen, D., Bista, M., Bradke, F., Jenne, D., Holak, T.A., Werb, Z., et al. (2008). Lifeact: a versatile marker to visualize F-actin. Nat Methods 5, 605-607.

Roberts, T.F., Tschida, K.A., Klein, M.E., and Mooney, R. (2010). Rapid spine stabilization and synaptic enhancement at the onset of behavioural learning. Nature 463, 948952.

Rochefort, N.L., Narushima, M., Grienberger, C., Marandi, N., Hill, D.N., and Konnerth, A. (2011). Development of direction selectivity in mouse cortical neurons. Neuron 71, 425-432.

Rose, T., Jaepel, J., Hubener, M., and Bonhoeffer, T. (2016). Cell-specific restoration of stimulus preference after monocular deprivation in the visual cortex. Science 352, 1319-1322.

Rothblat, L.A., Schwartz, M.L., and Kasdan, P.M. (1978). Monocular deprivation in the rat: evidence for an age-related defect in visual behavior. Brain Res 158, 456-460.

Ruano, D., Aulchenko, Y.S., Macedo, A., Soares, M.J., Valente, J., Azevedo, M.H., Hutz, M.H., Gama, C.S., Lobato, M.I., Belmonte-de-Abreu, P., et al. (2008). Association of the gene encoding neurogranin with schizophrenia in males. J Psychiatr Res 42, 125-133.

Rumpel, S., Kattenstroth, G., and Gottmann, K. (2004). Silent synapses in the immature visual cortex: layer-specific developmental regulation. J Neurophysiol 91, 10971101.

Ruska, E., and Knoll, M. (1931). Die magnetische Sammelspule für schnelle Elektronenstrahlen Z Techn Physik 12, 389-448.

Saito, T. (2006). In vivo electroporation in the embryonic mouse central nervous system. Nat Protoc 1, 1552-1558.

Saito, T., and Nakatsuji, N. (2001). Efficient gene transfer into the embryonic mouse brain using in vivo electroporation. Dev Biol 240, 237-246. 
Sajo, M., Ellis-Davies, G., and Morishita, H. (2016). Lynx1 Limits Dendritic Spine Turnover in the Adult Visual Cortex. J Neurosci 36, 9472-9478.

Sato, M., and Stryker, M.P. (2008). Distinctive features of adult ocular dominance plasticity. J Neurosci 28, 10278-10286.

Sawtell, N.B., Frenkel, M.Y., Philpot, B.D., Nakazawa, K., Tonegawa, S., and Bear, M.F. (2003). NMDA receptor-dependent ocular dominance plasticity in adult visual cortex. Neuron 38, 977-985.

Schindelin, J., Arganda-Carreras, I., Frise, E., Kaynig, V., Longair, M., Pietzsch, T., Preibisch, S., Rueden, C., Saalfeld, S., Schmid, B., et al. (2012). Fiji: an open-source platform for biological-image analysis. Nat Methods 9, 676-682.

Schlüter, O.M., Xu, W., and Malenka, R.C. (2006). Alternative N-terminal domains of PSD95 and SAP97 govern activity-dependent regulation of synaptic AMPA receptor function. Neuron 51, 99-111.

Schwarz, C., Hentschke, H., Butovas, S., Haiss, F., Stuttgen, M.C., Gerdjikov, T.V., Bergner, C.G., and Waiblinger, C. (2010). The head-fixed behaving rat--procedures and pitfalls. Somatosens Mot Res 27, 131-148.

Seaton, G., Hodges, G., de Haan, A., Grewal, A., Pandey, A., Kasai, H., and Fox, K. (2020). Dual-Component Structural Plasticity Mediated by alphaCaMKII Autophosphorylation on Basal Dendrites of Cortical Layer 2/3 Neurones. J Neurosci 40, 2228-2245.

Silva, A.J., Wang, Y., Paylor, R., Wehner, J.M., Stevens, C.F., and Tonegawa, S. (1992). Alpha calcium/calmodulin kinase II mutant mice: deficient long-term potentiation and impaired spatial learning. Cold Spring Harb Symp Quant Biol 57, 527-539.

Silver, M.A., and Stryker, M.P. (1999). Synaptic density in geniculocortical afferents remains constant after monocular deprivation in the cat. J Neurosci 19, 10829-10842.

Singec, I., Knoth, R., Ditter, M., Volk, B., and Frotscher, M. (2004). Neurogranin is expressed by principal cells but not interneurons in the rodent and monkey neocortex and hippocampus. J Comp Neurol 479, 30-42.

Stathakis, D.G., Hoover, K.B., You, Z., and Bryant, P.J. (1997). Human postsynaptic density-95 (PSD95): location of the gene (DLG4) and possible function in nonneural as well as in neural tissues. Genomics 44, 71-82.

Stein, V., House, D.R., Bredt, D.S., and Nicoll, R.A. (2003). Postsynaptic density-95 mimics and occludes hippocampal long-term potentiation and enhances long-term depression. J Neurosci 23, 5503-5506.

Stryker, M.P., and Löwel, S. (2018). Amblyopia: New molecular/pharmacological and environmental approaches. Vis Neurosci 35, E018. 
Sun, Y.J., Espinosa, J.S., Hoseini, M.S., and Stryker, M.P. (2019). Experience-dependent structural plasticity at pre- and postsynaptic sites of layer $2 / 3$ cells in developing visual cortex. Proc Natl Acad Sci U S A 116, 21812-21820.

Svirsky, S., Henchir, J., Li, Y., Ma, X., Carlson, S., and Dixon, C.E. (2020). Neurogranin Protein Expression Is Reduced after Controlled Cortical Impact in Rats. J Neurotrauma 37, 939-949.

Svoboda, K., Denk, W., Kleinfeld, D., and Tank, D.W. (1997). In vivo dendritic calcium dynamics in neocortical pyramidal neurons. Nature 385, 161-165.

Svoboda, K., Denk, W., Knox, W.H., and Tsuda, S. (1996). Two-photon-excitation scanning microscopy of living neurons with a saturable Bragg reflector mode-locked diodepumped Cr:LiSrAIFI laser. Opt Lett 21, 1411-1413.

Syken, J., Grandpre, T., Kanold, P.O., and Shatz, C.J. (2006). PirB restricts oculardominance plasticity in visual cortex. Science 313, 1795-1800.

Taft, C.E., and Turrigiano, G.G. (2014). PSD-95 promotes the stabilization of young synaptic contacts. Philos Trans R Soc Lond B Biol Sci 369, 20130134.

Torii, N., Kamishita, T., Otsu, Y., and Tsumoto, T. (1995). An inhibitor for calcineurin, FK506, blocks induction of long-term depression in rat visual cortex. Neurosci Lett 185, 1-4.

Trachtenberg, J.T., Chen, B.E., Knott, G.W., Feng, G., Sanes, J.R., Welker, E., and Svoboda, K. (2002). Long-term in vivo imaging of experience-dependent synaptic plasticity in adult cortex. Nature 420, 788-794.

Trachtenberg, J.T., Trepel, C., and Stryker, M.P. (2000). Rapid extragranular plasticity in the absence of thalamocortical plasticity in the developing primary visual cortex. Science 287, 2029-2032.

Uchizono, K. (1965). Characteristics of excitatory and inhibitory synapses in the central nervous system of the cat. Nature 207, 642-643.

Vidal, G.S., Djurisic, M., Brown, K., Sapp, R.W., and Shatz, C.J. (2016). Cell-Autonomous Regulation of Dendritic Spine Density by PirB. eNeuro 3.

Villa, K.L., Berry, K.P., Subramanian, J., Cha, J.W., Chan Oh, W., Kwon, H.B., Kubota, Y., So, P.T., and Nedivi, E. (2016). Inhibitory Synapses Are Repeatedly Assembled and Removed at Persistent Sites In Vivo. Neuron 90, 662-664.

Wang, B.S., Sarnaik, R., and Cang, J. (2010). Critical period plasticity matches binocular orientation preference in the visual cortex. Neuron 65, 246-256.

Wang, Y., Wu, W., Zhang, X., Hu, X., Li, Y., Lou, S., Ma, X., An, X., Liu, H., Peng, J., et al. (2016). A Mouse Model of Visual Perceptual Learning Reveals Alterations in Neuronal Coding and Dendritic Spine Density in the Visual Cortex. Front Behav Neurosci 10, 42. 
Watson, J.B., Sutcliffe, J.G., and Fisher, R.S. (1992). Localization of the protein kinase C phosphorylation/calmodulin-binding substrate RC3 in dendritic spines of neostriatal neurons. Proc Natl Acad Sci U S A 89, 8581-8585.

Webber, A.L., and Wood, J. (2005). Amblyopia: prevalence, natural history, functional effects and treatment. Clin Exp Optom 88, 365-375.

Wiesel, T.N., and Hubel, D.H. (1963a). Effects of Visual Deprivation on Morphology and Physiology of Cells in the Cats Lateral Geniculate Body. J Neurophysiol 26, 978993.

Wiesel, T.N., and Hubel, D.H. (1963b). Single-Cell Responses in Striate Cortex of Kittens Deprived of Vision in One Eye. J Neurophysiol 26, 1003-1017.

Yang, G., Chang, P.C., Bekker, A., Blanck, T.J., and Gan, W.B. (2011). Transient effects of anesthetics on dendritic spines and filopodia in the living mouse cortex. Anesthesiology 115, 718-726.

Yao, W.D., Gainetdinov, R.R., Arbuckle, M.I., Sotnikova, T.D., Cyr, M., Beaulieu, J.M., Torres, G.E., Grant, S.G., and Caron, M.G. (2004). Identification of PSD-95 as a regulator of dopamine-mediated synaptic and behavioral plasticity. Neuron $41,625-$ 638.

Yoshii, A., Sheng, M.H., and Constantine-Paton, M. (2003). Eye opening induces a rapid dendritic localization of PSD-95 in central visual neurons. Proc Natl Acad Sci U S A 100, 1334-1339.

Yu, H., Majewska, A.K., and Sur, M. (2011). Rapid experience-dependent plasticity of synapse function and structure in ferret visual cortex in vivo. Proc Natl Acad Sci $U$ S A 108, 21235-21240.

Yuste, R., and Bonhoeffer, T. (2001). Morphological changes in dendritic spines associated with long-term synaptic plasticity. Annu Rev Neurosci 24, 1071-1089.

Zhou, Y., Lai, B., and Gan, W.B. (2017). Monocular deprivation induces dendritic spine elimination in the developing mouse visual cortex. Sci Rep 7, 4977.

Zuo, Y., Yang, G., Kwon, E., and Gan, W.B. (2005). Long-term sensory deprivation prevents dendritic spine loss in primary somatosensory cortex. Nature 436, 261-265. 


\section{Abbreviations}

AAV : adeno-associated virus

AMPAR : a-amino-3-hydroxy-5-methyl-4-isoxazolepropionic acid receptor

BDNF : brain-derived neurotropic factor

CaM : calmodulin

CaMKII : calmodulin (CaM)-dependent protein kinase II

CCD : charge-coupled device

contra/ipsi : contralateral / ipsilateral eye

$\mathrm{CP}$ : critical period

CSPG : chondroitin sulfate proteoglycan

d : day

E : embryonic day

EM : electron microcopy

GABA : $y$-aminobutyric acid

GAD : glutamic acid decarboxylase

GFP : green fluorescent protein

GLME : generalized linear mixed-effects models

hr : hour

KD : knockdown

kDA: kilo Daltons

$\mathrm{KO}$ : knockout

$\mathrm{L}$ : layer

LME : linear mixed-effects models

LTD : long-term depression

LTP : long-term potentiation

MAGUK : membrane-associated guanylate kinases

MHC I : major histocompatibility complex class I

MD : monocular deprivation

$\mathrm{Ng}$ : neurogranin

NgR1 : Nogo receptor 1

NMDAR : N-methyl-D-aspartate receptor

$\mathrm{NV}$ : normal vision

OD : ocular dominance

ODP : ocular dominance plasticity

$\mathrm{P}$ : postnatal day

PirB : Paired immunoglobulin-like receptor 
PBS : phosphate buffered saline

$P N$ : pyramidal neuron

PSD : postsynaptic density

PV : parvalbumin

$\mathrm{RO}$ : reopening

SD : standard deviation

SEM : standard error of the mean

shRNA : small hairpin ribonucleic acid

shLc: shRNA luciferase

shNg : shRNA neurogranin

Tris-EDTA : tris(hydroxymethyl)aminomethane - ethylenediaminetetraacetic acid

V1 : primary visual cortex

VWT : visual water task

WT : wild-type

YFP : yellow fluorescent protein 


\section{Acknowledgements}

First of all, I would like to thank Prof. Dr. Siegrid Löwel for the opportunity to work in her laboratory and the continuous support to carry out and further develop the projects throughout my doctoral studies. She has let me be independent, but guided whenever I needed, while also supporting me to get involved in local and international scientific events.

I would like to thank Prof. Dr. Oliver Schlüter for his crucial contributions to my doctoral studies as part of my thesis committee, especially through critical and inspiring discussions which helped to develop my projects. I was also lucky to be able to use the facilities and equipment in Schlüter Lab, which was essential for timely completion of this thesis.

I thank Prof. Dr. Marion Silies for agreeing to be part of my thesis committee and for her much appreciated comments and advice during the meetings.

I thank Prof. Dr. Jochen Staiger for creating the opportunity to perform in utero electroporation in his lab, and also agreeing to evaluate my doctoral work. I thank Dr. Nieves Mingo Moreno for showing me the technique and all the other members of lab for allowing me to use their lab space and helping whenever needed.

I also thank Dr. Katrin Willig and Prof. Dr. Florentin Wörgötter for their interest in my thesis topic and agreeing to evaluate my work as part of the examination board.

All the members of Schlüter Lab, especially Sandra Ott-Gebauer, Dr. Plinio Favaro, Dr. Man Ho Wong, and Karl-Alexander Engelhardt have introduced me to the lab and helped with technical matters. I thank Myrto Panapoulou for her help with genotyping and warm hugs. Thank you all for making me feel very welcome in your lab!

I thank all the previous and current members of Löwel Lab-Kalina, Andrew, Susanne and Conni for a pleasant work environment and great time we spent in and outside the lab. I especially thank Evgenia for being a great friend and a colleague at the same time! I thank Anja for introducing me to two-photon microscopy and the cranial window surgeries, Simone, Matthias and Cedric for solving all bureaucratic/technical issues that I encountered. I thank Thomas Kräll and Josephine Böhner for helping me with some experiments during their bachelor thesis and lab rotations.

I thank 99\% Invisible for making my microscopy days more bearable with their awesome podcast, which surely helped me to produce this thesis in beautiful downtown Göttingen.

I am grateful to be part of the IMPRS Neuroscience program. I thank the coordination office, especially Sandra Drube, for taking care of everything for us. Through this program, I have met great people and made friends from all over the world. Sinem, Kanishka, Albert, Lukas, Myrto, Eric, Mar - thank you guys for all the memorable time we have spent together.

I also thank all my Azerbaijani friends living across different continents and countries, especially Elmir, Artoghrul, Nazakat, Agil, Sadig, Subhan and Toghrul, for keeping in touch all this time and visiting each other whenever possible.

I thank my family for never getting bored of asking me how the experiments with my mice are going on, and my brother Ismayil and cousin Elkhan for their friendly support.

Last but not least, I thank my girlfriend Müjgan, who always motivated and managed to cheer me up even if I had to be in the lab on an early Sunday morning - Mıkı, dur ! 